\title{
Simultaneous Collision of the Rigid Body at Two Points
}

\author{
Ionuț-Bogdan Dragna, Nicolae Pandrea and Nicolae-Doru Stănescu *(D) \\ Department of Manufacturing and Industrial Management, Faculty of Mechanics and Technology, \\ University of Pitești, 110040 Pitești, Romania; ionut.dragna@upit.ro (I.-B.D.); nicolae.pandrea@upit.ro (N.P.) \\ * Correspondence: doru.stanescu@upit.ro
}

check for updates

Citation: Dragna, I.-B.; Pandrea, N.; Stănescu, N.-D. Simultaneous Collision of the Rigid Body at Two Points. Symmetry 2021, 13, 1924. https://doi.org/10.3390/sym13101924

Academic Editor: Jan Awrejcewicz

Received: 13 September 2021

Accepted: 9 October 2021

Published: 13 October 2021

Publisher's Note: MDPI stays neutral with regard to jurisdictional claims in published maps and institutional affiliations.

\begin{abstract}
We present a new approach based on the notion of inertance for the simultaneous collisions without friction of a rigid solid. The calculations are performed using the screw (plückerian) coordinates, while the results are obtained in matrix form, and they may be easily implemented for different practical situations. One calculates the velocities after collision, the energy of lost velocities, and the loss of the kinetic energy. The general algorithm of calculation is described in the paper. The main assumption is that the normal velocities at the contact points vanish simultaneously. The coefficients of restitution at the contact points may be equal or not. Some completely solved applications are also presented, and the numerical results are discussed. The numerical values depend on which coefficient of restitution is used.
\end{abstract}

Keywords: simultaneous collision; coefficient of restitution; inertance; screw coordinates

\section{Introduction}

The problem of the collision of rigid bodies is often met in the literature. The main problems may be described as follows. Most papers deal with the collision at one point, the collision being without [1-14] or with friction [15-44]. Usually, the friction at the contact points is a Coulombian one [15-43], but other types of friction may be also considered [44]. The unanimous accepted hypotheses for the collisions are [1-49]: all the forces excepting the impact ones are neglected, there are two phases of collisions (compression and restitution), the rigid displacements vanish, and the tangential stiffness is infinite. Three different coefficients of restitution are used: Newton, Poisson, and energetic. It is proved $[14,47]$ that in the in the case of the collision without friction the three coefficients of restitution have equal values, but this is not the singular case for their equality [43]. Researchers [30,31,39-41,43] have proved that in the case of collision with friction, the three coefficients of restitution may have different values. Moreover [43], there exists no relation between the values of the three coefficients of restitution and it is possible [43,47] that some certain coefficients of restitution may have values greater than 1 in the general case of collision with friction. The authors [30,31,40-42] have proved that the consideration of the Newton or Poisson variants may lead to inconsistent results. Generally, the authors consider that the best results in the study of collisions with friction are obtained using the energetic coefficient of restitution [15-37,39-43], but one may use some particular coefficients of restitution [38].

Most researchers deal with collision at one point, regardless of whether they discuss singular collision, multiple successive collisions, or collisions of particular kinematic chains. The approaches are based on classical mechanics and use of Lagrange's or Kane's equations. The simultaneous collision of a rigid solid at several points implies some difficulties concerning the distribution of velocities after the collision. This distribution must satisfy the Euler equation for the velocities of a rigid solid. Some aspects are discussed in [38-41] for some particular cases considering that at both points of collision, the coefficients of restitution are equal. The collision with friction may lead to complex mathematical problems concerning the cases of sticking and slipping of the rigid solid. It is proved 
that, in this situation, the contact point may move only on maximum four principal directions [43] resulting a system of polynomial equations [39-41] for which the solutions are obtained by numerical methods [49]. The phenomenon of jamb [24] is generally avoided in the references.

In our paper, we deal with screw coordinates [14,43,45-49]. The notion of inertance was introduced by Pandrea [13,48] and developed by Pandrea et al. [14,43,49].

Aside from the classical hypotheses at collision we added a supplementary one that states that the normal velocities at the contact points vanish simultaneously.

The paper is organized as follows: in Section 2, we present the case of the free rigid solid acted by two impulses at two different points; Sections 3 and 4 are dedicated to the calculations of the velocities after collision, the energy of lost velocities, and the loss of kinetic energy; in Section 5, we highlight some particular cases; Section 6 describes the algorithm of calculation for the collision of the free rigid solid at two points; Section 7 is reserved for the presentation of four numerical applications, while in Section 8, we perform some discussions concerning the collision of the free rigid solid at two points; in Section 9, we discuss the general case of the collision at two points of the constrained rigid solid, and in Section 10, we calculate the velocities after collision; Section 11 is dedicated to the presentation of three numerical applications concerning the collision at two points of a rigid solid with constraints; in Section 12, we present the algorithm for the collision at two points of the rigid solid bounded by the surface of a certain equation, and in Section 13, we discuss an example; in Section 14, we present some general aspects concerning the collision with Coulombian friction; the paper ends with a Conclusion section.

\section{The Case of the Free Rigid Solid}

\subsection{General Formulation of the Problem}

We have a rigid solid in general motion, and one considers the system $C x y z$ of the central principal axes of inertia, $C$ being the center of weight. The kinematical parameters before the collision are $\mathbf{v}_{C}^{0}$ (the velocity of the center of mass) and $\boldsymbol{\omega}^{0}$ (the angular velocity) (Figure 1). The rigid solid collides normally at the points $A_{1}$ and $A_{2}$, the collisions being without friction.

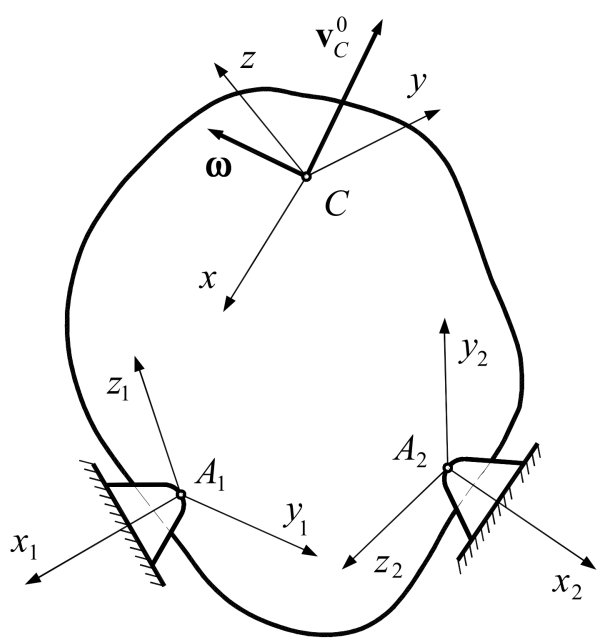

Figure 1. The formulation of the problem.

One considers as known:

- $\quad$ the mass $m$ and the central principal moments of inertia $J_{x}, J_{y}$, and $J_{z}$ for the rigid solid;

- the restitution coefficients $k_{N}, k_{P}$, and $k_{e}$, respectively, corresponding to the Newton, Poisson and energetic variants, respectively, defined by the relations $k_{N}=-\frac{v_{12 n}^{f}}{v_{12 n}^{0}}$, $k_{P}=\frac{P_{d}}{P_{c}}$, and $k_{e}=\sqrt{\frac{\left|\Delta E_{c}^{d}\right|}{\Delta E_{c}^{c}}}$, respectively, are identical [14] in the case of a single colli- 
sion without friction. In the previous expressions, $v_{12 n}^{f}$ represents the relative normal velocity after the collision at the contact point, $v_{12 n}^{0}$ is the relative normal velocity before the collision at the contact point, $P_{d}$ is the impulse in the phase of restitution, $P_{c}$ is the impulse corresponding to the phase of compression, $\Delta E_{c}^{d}$ represents the variation of the kinetic energy in the phase of restitution, and $\Delta E_{c}^{c}$ is the variation of the kinetic energy in the phase of compression;

- the normal axes orientated to the outside of the rigid solid $A_{1} x_{1}$ and $A_{2} x_{2}$ at the collision points $A_{1}$ and $A_{2}$, respectively;

- the status of velocity $\boldsymbol{\omega}^{0}$ and $\mathbf{v}_{C}^{0}$ of the rigid solid before the collision.

One asks for (at the end of collision):

- $\quad$ the velocity $\mathbf{v}_{C}$ and the angular velocity $\boldsymbol{\omega}$;

- $\quad$ the velocities at the collision points;

- $\quad$ the magnitudes $P_{1}$ and $P_{2}$ of the impulses at the points $A_{1}$ and $A_{2}$, respectively;

- $\quad$ the kinetic energy of the lost velocities;

- $\quad$ the loss of kinetic energy.

One considers the cases of the Newton and Poisson variants.

The main hypothesis states that the normal velocities at the points $A_{1}$ and $A_{2}$ simultaneously vanish.

\subsection{Notations}

We use the following notations:

- the components $\omega_{x}^{0}, \omega_{y}^{0}, \omega_{z}^{0}$ and $\omega_{x}, \omega_{y}, \omega_{z}$ of the angular velocities $\omega^{0}$ and $\omega$, respectively;

- the components $v_{C x}^{0}, v_{C y}^{0}$, and $v_{C z}^{0}$ and $v_{C x}, v_{C y}$, and $v_{C z}$ of the velocities $\mathbf{v}_{C}^{0}$ and $\mathbf{v}_{C}$, respectively;

- the screw (plückerian) coordinates of the velocities of the rigid solid

$$
\begin{aligned}
& \left\{\mathbf{v}_{C}^{0}\right\}=\left[\begin{array}{llllll}
\omega_{x}^{0} & \omega_{y}^{0} & \omega_{z}^{0} & v_{C x}^{0} & v_{C y}^{0} & v_{C z}^{0}
\end{array}\right]^{\mathrm{T}}, \\
& \left\{\mathbf{v}_{C}\right\}=\left[\begin{array}{llllll}
\omega_{x} & \omega_{y} & \omega_{z} & v_{C x} & v_{C y} & v_{C z}
\end{array}\right]^{\mathrm{T}} ;
\end{aligned}
$$

- $\quad$ the coordinates $x_{1}, y_{1}$, and $z_{1}$ and $x_{2}, y_{2}$, and $z_{2}$ of the points $A_{1}$ and $A_{2}$, respectively, relative to the reference frame $C x y z$;

- $\quad$ the director cosines of the axes of the local reference systems $A_{1} x_{1} y_{1} z_{1}$ and $A_{2} x_{2} y_{2} z_{2}$ and the rotational matrices $\left[\mathbf{R}_{1}\right]$ and $\left[\mathbf{R}_{2}\right]$

$$
\left[\mathbf{R}_{1}\right]=\left[\begin{array}{lll}
a_{11} & a_{12} & a_{13} \\
b_{11} & b_{12} & b_{13} \\
c_{11} & c_{12} & c_{13}
\end{array}\right],\left[\mathbf{R}_{2}\right]=\left[\begin{array}{lll}
a_{21} & a_{22} & a_{23} \\
b_{21} & b_{22} & b_{23} \\
c_{21} & c_{22} & c_{23}
\end{array}\right] ;
$$

- the matrices $\left[\mathbf{S}_{1}\right],\left[\mathbf{S}_{2}\right],\left[\mathbf{D}_{1}\right]$, and $\left[\mathbf{D}_{2}\right]$ defined by the relations

$$
\left[\mathbf{S}_{i}\right]=\left[\begin{array}{ccc}
0 & -z_{i} & y_{i} \\
z_{i} & 0 & -x_{i} \\
-y_{i} & x_{i} & 0
\end{array}\right],\left[\mathbf{D}_{i}\right]=\left[\mathbf{S}_{i}\right]\left[\mathbf{R}_{i}\right]=\left[\begin{array}{ccc}
d_{i 1} & d_{i 2} & d_{i 3} \\
e_{i 1} & e_{i 2} & e_{i 3} \\
f_{i 1} & f_{i 2} & f_{i 3}
\end{array}\right],
$$

with $i=1,2$;

- $\quad$ the matrices of the screw (plückerian) coordinates of the axes $A_{i} x_{i}, A_{i} y_{i}$, and $A_{i} z_{i}$, $i=1,2$,

$$
\begin{aligned}
& \left\{\mathbf{E}_{i x}\right\}=\left[\begin{array}{llllll}
a_{i 1} & b_{i 1} & c_{i 1} & d_{i 1} & e_{i 1} & f_{i 1}
\end{array}\right]^{\mathrm{T}}, \\
& \left\{\mathbf{E}_{i y}\right\}=\left[\begin{array}{llllll}
a_{i 2} & b_{i 2} & c_{i 2} & d_{i 2} & e_{i 2} & f_{i 2}
\end{array}\right]^{\mathrm{T}}, \\
& \left\{\mathbf{E}_{i z}\right\}=\left[\begin{array}{llllll}
a_{i 3} & b_{i 3} & c_{i 3} & d_{i 3} & e_{i 3} & f_{i 3}
\end{array}\right]^{\mathrm{T}},
\end{aligned}
$$

- $\quad$ the scalar values of the impulses $P_{1}$ and $P_{2}$ at the points $A_{1}$ and $A_{2}$, respectively; 
the matrix $[\eta]$ given by

$$
[\eta]=\left[\begin{array}{cc}
{[0]} & {[\mathbf{I}]} \\
{[\mathbf{I}]} & {[\mathbf{0}}
\end{array}\right]=\left[\begin{array}{llllll}
0 & 0 & 0 & 1 & 0 & 0 \\
0 & 0 & 0 & 0 & 1 & 0 \\
0 & 0 & 0 & 0 & 0 & 1 \\
1 & 0 & 0 & 0 & 0 & 0 \\
0 & 1 & 0 & 0 & 0 & 0 \\
0 & 0 & 1 & 0 & 0 & 0
\end{array}\right] ;
$$

- the $\operatorname{sign} *$ for the mixed product of two matrices in screw (plückerian) coordinates

$$
\left\{\mathrm{E}_{i x}\right\} *\left\{\mathrm{E}_{i y}\right\}=\left\{\mathrm{E}_{i x}\right\}^{\mathrm{T}}[\mathrm{\eta}]\left\{\mathbf{E}_{i y}\right\} ;
$$

- $\quad$ the matrices of position and their inverses

$$
\left[\mathbf{T}_{i}\right]=\left[\begin{array}{cc}
{\left[\mathbf{R}_{i}\right]} & {[\mathbf{0}]} \\
{\left[\mathbf{D}_{i}\right]} & {\left[\mathbf{R}_{i}\right]}
\end{array}\right],\left[\mathbf{T}_{i}\right]^{-\mathbf{1}}=\left[\begin{array}{cc}
{\left[\mathbf{R}_{i}\right]^{\mathrm{T}}} & {[\mathbf{0}]} \\
{\left[\mathbf{D}_{i}\right]^{\mathrm{T}}} & {\left[\mathbf{R}_{i}\right]^{\mathrm{T}}}
\end{array}\right],
$$

where $i=1,2$;

- $\quad$ the components $v_{1 x}, v_{1 y}$, and $v_{1 z}$ and $v_{2 x}, v_{2 y}$, and $v_{2 z}$ of the velocities at the points $A_{1}$ and $A_{2}$, respectively, components [48,49] given by relations

$$
v_{i x}=\left\{\mathrm{E}_{i x}\right\}^{\mathrm{T}}[\mathrm{\eta}]\left\{\mathbf{v}_{C}\right\}, v_{i y}=\left\{\mathrm{E}_{i y}\right\}^{\mathrm{T}}[\eta]\left\{\mathbf{v}_{C}\right\}, v_{i z}=\left\{\mathrm{E}_{i z}\right\}^{\mathrm{T}}[\eta]\left\{\mathbf{v}_{C}\right\},
$$

with $i=1$, ;

- the matrix of inertia of the rigid solid and its inverse

$$
\begin{gathered}
{[\mathbf{M}]=\left[\begin{array}{cc}
{[0]} & {[\mathbf{m}]} \\
{[\mathbf{J}]} & {[0]}
\end{array}\right]=\left[\begin{array}{cccccc}
0 & 0 & 0 & m & 0 & 0 \\
0 & 0 & 0 & 0 & m & 0 \\
0 & 0 & 0 & 0 & 0 & m \\
J_{x} & 0 & 0 & 0 & 0 & 0 \\
0 & J_{y} & 0 & 0 & 0 & 0 \\
0 & 0 & J_{z} & 0 & 0 & 0
\end{array}\right],} \\
{[\mathbf{M}]^{-1}=\left[\begin{array}{cc}
{[0]} & {[\mathrm{J}]^{-1}} \\
{[\mathrm{~m}]^{-1}} & {[0]}
\end{array}\right]=\left[\begin{array}{cccccc}
0 & 0 & 0 & J_{x}^{-1} & 0 & 0 \\
0 & 0 & 0 & 0 & J_{y}^{-1} & 0 \\
0 & 0 & 0 & 0 & 0 & J_{z}^{-1} \\
m^{-1} & 0 & 0 & 0 & 0 & 0 \\
0 & m^{-1} & 0 & 0 & 0 & 0 \\
0 & 0 & m^{-1} & 0 & 0 & 0
\end{array}\right] .}
\end{gathered}
$$

\subsection{Determination of the Impulses}

The general theorems at collisions $[13,14,48,49]$ may be put in the matrix relation

$$
\{\mathbf{M}\}\left\{\left\{\mathbf{v}_{C}\right\}-\left\{\mathbf{v}_{C}^{0}\right\}\right\}=-P_{1}\left\{\mathbf{E}_{1 x}\right\}-P_{2}\left\{\mathbf{E}_{2 x}\right\},
$$

wherefrom it results in

$$
\left\{\mathbf{v}_{C}\right\}-\left\{\mathbf{v}_{C}^{0}\right\}=-P_{1}[\mathbf{M}]^{-1}\left\{\mathbf{E}_{1 x}\right\}-P[\mathbf{M}]^{-1}{ }_{2}\left\{\mathbf{E}_{2 x}\right\} .
$$

Defining the inertances $[13,14,43,48,49]$ by the relations

$$
g_{i j x y}=\left\{\mathbf{E}_{i x}\right\}^{\mathrm{T}}[\boldsymbol{\eta}][\mathbf{M}]^{-1}\left\{\mathbf{E}_{j y}\right\}=g_{i j x y}, i=1,2,
$$


from the expression (11), one obtains

$$
v_{1 x}-v_{1 x}^{0}=-P_{1} g_{11 x x}-P_{2} g_{12 x x}, v_{2 x}-v_{2 x}^{0}=-P_{1} g_{21 x x}-P_{2} g_{22 x x} .
$$

2.3.1. The Newton Model

For the Newton model $[13,14,48,49]$, one may write

$$
v_{i x}=-k_{i}^{N} v_{i x}^{0}, i=1,2,
$$

and from (13), it results in

$$
\left(1+k_{1}^{N}\right) v_{1 x}^{0}=P_{1} g_{11 x x}+P_{2} g_{12 x x},\left(1+k_{2}^{N}\right) v_{2 x}^{0}=P_{1} g_{21 x x}+P_{2} g_{22 x x} .
$$

The situation is captured in Figure 2.

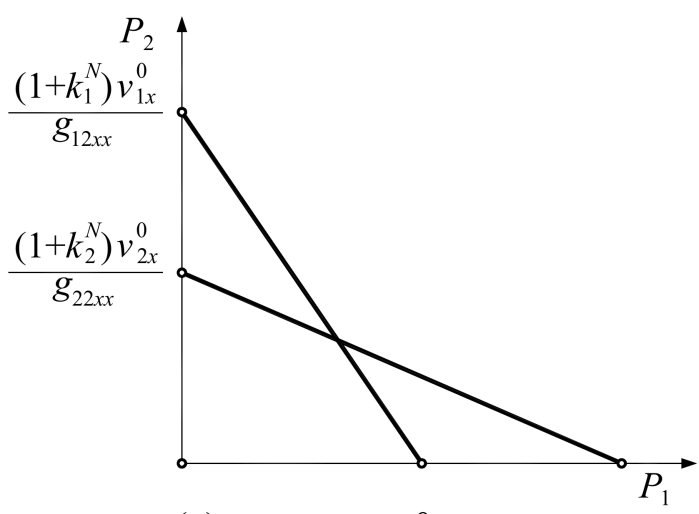

(a) $g_{12 x x}=g_{21 x x}>0$

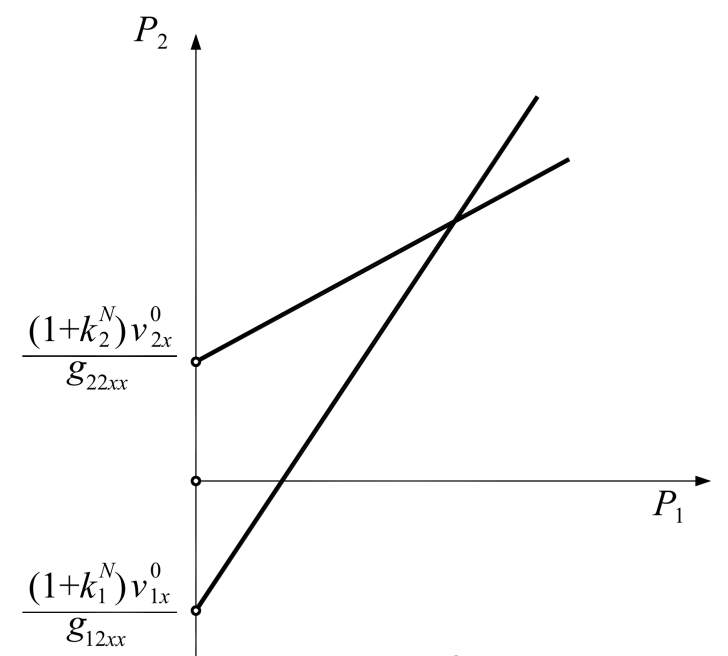

(b) $g_{12 x x}=g_{21 x x}<0$

Figure 2. (a) $g_{12 x x}=g_{21 x x}>0$; (b) $g_{12 x x}=g_{21 x x}<0$.

For the planar collision, one has

$$
\left\{\mathbf{E}_{1 x}\right\}=\left[\begin{array}{llllll}
a_{11} & b_{11} & 0 & 0 & 0 & f_{11}
\end{array}\right]^{\mathrm{T}},\left\{\mathbf{E}_{2 x}\right\}=\left[\begin{array}{llllll}
a_{21} & b_{21} & 0 & 0 & 0 & f_{21}
\end{array}\right]^{\mathrm{T}},
$$

with $a_{i 1}^{2}+b_{i 1}^{2}=1, i=1,2$.

It results in

$$
\begin{gathered}
g_{11 x x}=\frac{1}{m}+\frac{f_{11}^{2}}{J_{z}}, g_{12 x x}=\frac{a_{11} a_{21}+b_{11} b_{21}}{m}+\frac{f_{11} f_{21}}{J_{z}}=g_{21 x x}, g_{22 x x}=\frac{1}{m}+\frac{f_{21}^{2}}{J_{z}} \\
\left(1+k_{1}^{N}\right) v_{1 x}^{0}=P_{1} g_{11 x x}+P_{2} g_{12 x x},\left(1+k_{2}^{N}\right) v_{2 x}^{0}=P_{1} g_{12 x x}+P_{2} g_{22 x x} .
\end{gathered}
$$


The velocities after collision read

$\omega=\omega^{0}-P_{1} \frac{f_{11}}{m}-P_{2} \frac{f_{21}}{J_{z}}, v_{x}=v_{x}^{0}-P_{1} \frac{f_{11}}{m}-P_{2} \frac{f_{21}}{m}, v_{y}=v_{y}^{0}-P_{1} \frac{b_{11}}{m}-P_{2} \frac{b_{21}}{m}$.

In addition, one has

$$
\begin{aligned}
& v_{1 x}^{0}=a_{11} v_{x}^{0}+b_{11} v_{y}^{0}+f_{11} \omega^{0}, v_{1 x}=a_{11} v_{x}+b_{11} v_{y}+f_{11} \omega, \\
& v_{2 x}^{0}=a_{21} v_{x}^{0}+b_{21} v_{y}^{0}+f_{21} \omega^{0}, v_{2 x}=a_{21} v_{x}+b_{21} v_{y}+f_{21} \omega .
\end{aligned}
$$

Using the relations (15), one obtains the values of the impulses after collision for the Newton model

$$
P_{1}=\frac{\left|\begin{array}{cc}
\left(1+k_{1}^{N}\right) v_{1 x}^{0} & g_{12 x x} \\
\left(1+k_{2}^{N}\right) v_{2 x}^{0} & g_{22 x x}
\end{array}\right|}{\left|\begin{array}{ll}
g_{11 x x} & g_{12 x x} \\
g_{21 x x} & g_{22 x x}
\end{array}\right|}, P_{2}=\frac{\left|\begin{array}{ll}
g_{11 x x} & \left(1+k_{1}^{N}\right) v_{1 x}^{0} \\
g_{21 x x} & \left(1+k_{2}^{N}\right) v_{2 x}^{0}
\end{array}\right|}{\left|\begin{array}{ll}
g_{11 x x} & g_{12 x x} \\
g_{21 x x} & g_{22 x x}
\end{array}\right|} .
$$

\subsubsection{The Poisson Model}

The impulses $P_{i}^{c}, i=1,2$, in the compression phase are obtained from the relations (13) imposing the conditions $v_{i x}=0, i=1,2$; it results in the system

$$
v_{1 x}^{0}=P_{1}^{c} g_{11 x x}+P_{2}^{c} g_{12 x x}, v_{2 x}^{0}=P_{1}^{c} g_{21 x x}+P_{2}^{c} g_{22 x x}
$$

with the solutions

$$
P_{1}^{c}=\frac{\left|\begin{array}{ll}
v_{1 x}^{0} & g_{12 x x} \\
v_{2 x}^{0} & g_{22 x x}
\end{array}\right|}{\left|\begin{array}{ll}
g_{11 x x} & g_{12 x x} \\
g_{21 x x} & g_{22 x x}
\end{array}\right|}, P_{2}^{c}=\frac{\left|\begin{array}{ll}
g_{11 x x} & v_{1 x}^{0} \\
g_{21 x x} & v_{2 x}^{0}
\end{array}\right|}{\left|\begin{array}{ll}
g_{11 x x} & g_{12 x x} \\
g_{21 x x} & g_{22 x x}
\end{array}\right|}
$$

The total impulses are deduced from the relations

$$
P_{1}=\left(1+k_{1}^{P}\right) P_{1}^{c}, P_{2}=\left(1+k_{2}^{P}\right) P_{2}^{c}
$$

\section{Velocities after Collision}

The matrix of the screw (plückerian) coordinates of the velocity at the point $C$ is deduced from the relation (11)

$$
\left\{\mathbf{v}_{C}\right\}=\left\{\mathbf{v}_{C}^{0}\right\}-P_{1}[\mathbf{M}]^{-1}\left\{\mathbf{E}_{1 x}\right\}-P_{2}[\mathbf{M}]^{-1}\left\{\mathbf{E}_{2 x}\right\} .
$$

The tangential velocities at the point $A_{1}$ are

$$
v_{1 y}=v_{1 y}^{0}-P_{1} g_{11 y x}-P_{2} g_{12 y x}, v_{1 z}=v_{1 z}^{0}-P_{1} g_{11 z x}-P_{2} g_{12 z x},
$$

while the tangential velocities at the point $A_{2}$ read

$$
v_{2 y}=v_{2 y}^{0}-P_{1} g_{21 y x}-P_{2} g_{22 y x}, v_{2 z}=v_{2 z}^{0}-P_{1} g_{21 z x}-P_{2} g_{22 z x} .
$$

The angles $\alpha_{1}^{0}, \alpha_{1}, \alpha_{2}^{0}, \alpha_{2}$ between the tangential velocities and the axes $A_{i} y_{i}, i=1,2$, before and after the collision are obtained from the expression

$$
\begin{aligned}
\cos \alpha_{i}^{0}=\frac{v_{i y}^{0}}{\sqrt{\left(v_{i y}^{0}\right)^{2}+\left(v_{i z}^{0}\right)^{2}}}, \sin \alpha_{i}^{0}=\frac{v_{i z}^{0}}{\sqrt{\left(v_{i y}^{0}\right)^{2}+\left(v_{i z}^{0}\right)^{2}}}, \cos \alpha_{i}=\frac{v_{i y}}{\sqrt{v_{i y}^{2}+v_{i z}^{2}}}, \\
\sin \alpha_{i}=\frac{v_{i z}}{\sqrt{v_{i y}^{2}+v_{i z}^{2}}} .
\end{aligned}
$$




\section{The Energy of Lost Velocities $E_{p}$ and the Loose of Kinetic Energy $\Delta E_{c}$}

The energy of lost velocities $E_{p}[13,14,48,49]$ is defined by relation

$$
E_{p}=\frac{1}{2}\left\{\left\{\mathbf{v}_{c}\right\}-\left\{\mathbf{v}_{c}^{0}\right\}\right\}^{\mathrm{T}}[\boldsymbol{\eta}][\mathbf{M}]\left\{\left\{\mathbf{v}_{c}\right\}-\left\{\mathbf{v}_{c}^{0}\right\}\right\} .
$$

Taking into account the expressions (10) and (11), one deduces

$$
E_{p}=\frac{1}{2}\left\{P_{1}\left\{\mathbf{E}_{1 x}\right\}^{\mathrm{T}}+P_{2}\left\{\mathbf{E}_{2 x}\right\}^{\mathrm{T}}\right\}\left[[\mathbf{M}]^{-1}\right]^{\mathrm{T}}[\mathbf{\eta}]\left\{P_{1}\left\{\mathbf{E}_{1 x}\right\}+P_{2}\left\{\mathbf{E}_{2 x}\right\}\right\} .
$$

On the other hand,

$$
\left[[\mathbf{M}]^{-1}\right]^{\mathrm{T}}[\mathbf{\eta}]=[\mathbf{\eta}]\left[[\mathbf{M}]^{-1}\right]
$$

and the relations (12) lead to

$$
E_{p}=\frac{1}{2}\left(P_{1}^{2} g_{11 x x}+2 P_{1} P_{2} g_{12 x x}+P_{2}^{2} g_{22 x x}\right) .
$$

The loss of kinetic energy

$$
\Delta E_{\mathcal{C}}=\frac{1}{2}\left\{\mathbf{v}_{C}^{0}\right\}^{\mathrm{T}}[\boldsymbol{\eta}][\mathbf{M}]\left\{\mathbf{v}_{C}^{0}\right\}-\frac{1}{2}\left\{\mathbf{v}_{c}\right\}^{\mathrm{T}}[\boldsymbol{\eta}][\mathbf{M}]\left\{\mathbf{v}_{C}\right\}
$$

is obtained by considering the expression (28), which successively reads

$$
\begin{aligned}
E_{p} & =\frac{1}{2}\left\{\mathbf{v}_{C}\right\}^{\mathrm{T}}[\boldsymbol{\eta}][\mathbf{M}]\left\{\mathbf{v}_{C}\right\}-\left\{\mathbf{v}_{C}\right\}^{\mathrm{T}}[\boldsymbol{\eta}][\mathbf{M}]\left\{\mathbf{v}_{C}^{0}\right\}+\frac{1}{2}\left\{\mathbf{v}_{C}^{0}\right\}^{\mathrm{T}}[\boldsymbol{\eta}][\mathbf{M}]\left\{\mathbf{v}_{C}^{0}\right\}= \\
& =-\Delta E_{C}-\left\{\mathbf{v}_{C}^{0}\right\}^{\mathrm{T}}[\boldsymbol{\eta}][\mathbf{M}]\left\{\left\{\mathbf{v}_{C}\right\}-\left\{\mathbf{v}_{C}^{0}\right\}\right\}=-\Delta E_{\mathcal{C}}+P_{1} v_{1 x}^{0}+P_{2} v_{2 x}^{0} ;
\end{aligned}
$$

yielding

$$
\Delta E_{c}=-E_{p}+P_{1} v_{1 x}^{0}+P_{2} v_{2 x}^{0}
$$

\section{Particular Cases}

\section{1. $g_{12 x x}=0$}

If the collision directions are perpendicular, and one of them passes through the center, then $g_{12 x x}=0$; it results in the final impulses

$$
P_{1}=\frac{\left(1+k_{1}^{N}\right) v_{1 x}^{0}}{g_{11 x x}}, P_{2}=\frac{\left(1+k_{2}^{N}\right) v_{2 x}^{0}}{g_{22 x x}}
$$

for the Newton model, and

$$
P_{1}=\frac{\left(1+k_{1}^{P}\right) v_{1 x}^{0}}{g_{11 x x}}, P_{2}=\frac{\left(1+k_{2}^{P}\right) v_{2 x}^{0}}{g_{22 x x}}
$$

for the Poisson model, respectively.

5.2. $\left[\boldsymbol{R}_{1}\right]=\left[\boldsymbol{R}_{2}\right]=[\boldsymbol{I}]$

In this situation, one obtains

$$
\begin{gathered}
{\left[\mathbf{D}_{i}\right]=\left[\mathbf{S}_{i}\right]=\left[\begin{array}{ccc}
0 & -z_{i} & y_{i} \\
z_{i} & 0 & -x_{i} \\
-y_{i} & x_{i} & 0
\end{array}\right], i=1,2,} \\
\left\{\mathbf{E}_{1 x}\right\}=\left[\begin{array}{llllll}
1 & 0 & 0 & 0 & z_{1} & -y_{1}
\end{array}\right]^{\mathrm{T}},\left\{\mathbf{E}_{2 x}\right\}=\left[\begin{array}{llllll}
1 & 0 & 0 & 0 & z_{2} & -y_{2}
\end{array}\right]^{\mathrm{T}}, \\
g_{11 x x}=\frac{1}{m}+\frac{z_{1}^{2}}{J_{y}}+\frac{y_{1}^{2}}{J_{z}}, g_{12 x x}=\frac{1}{m}+\frac{z_{1} z_{2}}{J_{y}}+\frac{y_{1} y_{2}}{J_{z}}, g_{22 x x}=\frac{1}{m}+\frac{z_{2}^{2}}{J_{y}}+\frac{y_{2}^{2}}{J_{z}} .
\end{gathered}
$$




\subsection{Planar Case}

Let us return to the planar case, where we obtained the system

$$
\left(1+k_{1}^{N}\right) v_{1 x}^{0}=P_{1} g_{11 x x}+P_{2} g_{12 x x},\left(1+k_{2}^{N}\right) v_{2 x}^{0}=P_{1} g_{21 x x}+P_{2} g_{22 x x},
$$

that is, a system of two linear equations with two unknowns.

Assuming that $k_{1}^{N}=k_{2}^{N}, v_{1 x}^{0}=v_{2 x}^{0}$, and $P_{1}=P_{2}=P$, the system (40) becomes

$$
P\left(g_{11 x x}+g_{12 x x}\right)=A, P\left(g_{21 x x}+g_{22 x x}\right)=A,
$$

where

$$
A=\left(1+k_{1}^{N}\right) v_{1 x}^{0}=\left(1+k_{2}^{N}\right) v_{2 x}^{0} .
$$

The system (41) has a unique solution if and only if

$$
g_{11 x x}+g_{12 x x}=g_{21 x x}+g_{22 x x} \neq 0 .
$$

The conditions (43) imply

$$
\frac{1}{m}+\frac{f_{11}^{2}}{J_{z}}=\frac{1}{m}+\frac{f_{21}^{2}}{J_{z}}, f_{11}= \pm f_{21} .
$$

If we consider that the rotational matrices are

$$
\left[\mathbf{R}_{1}\right]=\left[\mathbf{R}_{2}\right]=[\mathbf{I}],
$$

then

$$
\begin{aligned}
a_{11}=1, a_{21}=1, b_{11} & =0, b_{21}=0, f_{11}=-a_{11} y_{1}+b_{11} x_{1}=-y_{1}, \\
f_{21} & =-a_{21} y_{2}+b_{21} x_{2}=-y_{2}
\end{aligned}
$$

and the relations (44) lead us to

$$
\begin{gathered}
y_{1}= \pm y_{2}, \\
\frac{2}{m}+\frac{y_{1}\left(y_{1}+y_{2}\right)}{J_{z}} \neq 0 .
\end{gathered}
$$

No matter the value of $y_{1}\left(y_{1}=y_{2}\right.$ or $\left.y_{1}=-y_{2}\right)$, the relation (48) always holds true.

One may observe that the conditions $k_{1}^{N}=k_{2}^{N}$ and $v_{1 x}^{0}=v_{2 x}^{0}$ can be replaced by a weaker condition, namely

$$
\frac{2}{m}+\frac{y_{1}\left(y_{1}+y_{2}\right)}{J_{z}} \neq 0
$$

\section{Algorithm of Calculation}

The input data are:

- mechanical and geometrical characteristics of the rigid solid $\left(m, C, J_{x}, J_{y}\right.$, and $\left.J_{z}\right)$;

- $\quad$ the two points of collision $A_{1}$ and $A_{2}$, that is the coordinates $x_{i}, y_{i}$, and $z_{i}$, with $i=\overline{1,2}$, relative to the reference frame $C x y z$;

- $\quad$ the velocities $\mathbf{v}_{C}^{0}$ and $\boldsymbol{\omega}^{0}$ before collision;

- $\quad$ the director cosines of the axes of the local systems $A_{i} x_{i} y_{i} z_{i}, i=\overline{1,2}$;

- $\quad$ the coefficients of restitution $k_{1}$ and $k_{2}$.

One successively calculates:

- the rotational matrices $\left[\mathbf{R}_{1}\right]$ and $\left[\mathbf{R}_{2}\right]$ using the expressions (2);

- $\quad$ the matrices $\left[\mathbf{S}_{i}\right]$ and $\left[\mathbf{D}_{i}\right], i=\overline{1,2}$, using the formulae (3);

- the matrices of screw coordinates $\left\{\mathbf{E}_{i x}\right\},\left\{\mathbf{E}_{i y}\right\}$ and $\left\{\mathbf{E}_{i z}\right\}, i=\overline{1,2}$, using the relations (8);

- $\quad$ the matrix $[\boldsymbol{\eta}]$ given by (5); 
- $\quad$ the values $v_{i x}, v_{i y}$ and $v_{i z}, i=\overline{1,2}$, using the formulae (8);

- $\quad$ the matrix of inertia $[\mathbf{M}]$ and its inverse $[\mathbf{M}]^{-1}$ with the aid of the expression (9);

- $\quad$ the inertances $g_{i j x x}, i, j=\overline{1,2}$, using the formulae (12);

- $\quad$ the impulses $P_{1}$ and $P_{2}$ :

(a) for the Newton model using the relations (16),

(b) for the Poisson model using the expression (18) and (19);

- $\quad$ the velocities after the collision with the aid of the expressions (24)-(26);

- $\quad$ the energy of lost velocities $E_{p}$ with the relation (28);

- $\quad$ the loss of kinetic energy using the expression (34).

\section{Numerical Applications}

Example 1. The collision of a sphere at two points (Figure 3).

Due to the symmetry, we may choose the reference frames as shown in Figure 3. In addition: $J_{x}=J_{z}=J, x_{1}=R, y_{1}=0, z_{1}=0, z_{2}=0, x_{2}=R \cos \theta$, and $y_{2}=R \sin \theta$, where $R$ is the radius of the sphere, and $\theta$ is the angle between the axis $C x_{2} \equiv A_{2} x_{2}$ and the fixed axis $C x_{1} \equiv A_{1} x_{1}, \mathbf{v}_{C}^{0}=\omega_{0} R \mathbf{i}, \omega^{0}=\omega_{0} \mathbf{k}, k_{i}^{N}=k_{0}^{P}=k$, and $i=\overline{1,2}$.

It results in the following

$$
\begin{gathered}
{\left[\mathbf{R}_{1}\right]=\left[\begin{array}{lll}
1 & 0 & 0 \\
0 & 1 & 0 \\
0 & 0 & 1
\end{array}\right],\left[\mathbf{R}_{2}\right]=\left[\begin{array}{ccc}
\cos \theta & -\sin \theta & 0 \\
\sin \theta & \cos \theta & 0 \\
0 & 0 & 1
\end{array}\right],} \\
{\left[\mathbf{S}_{1}\right]=\left[\begin{array}{ccc}
0 & 0 & 0 \\
0 & 0 & -R \\
0 & R & 0
\end{array}\right],\left[\mathbf{S}_{2}\right]=\left[\begin{array}{ccc}
0 & 0 & R \sin \theta \\
0 & 0 & -R \cos \theta \\
-R \sin \theta & R \cos \theta & 0
\end{array}\right],}
\end{gathered}
$$

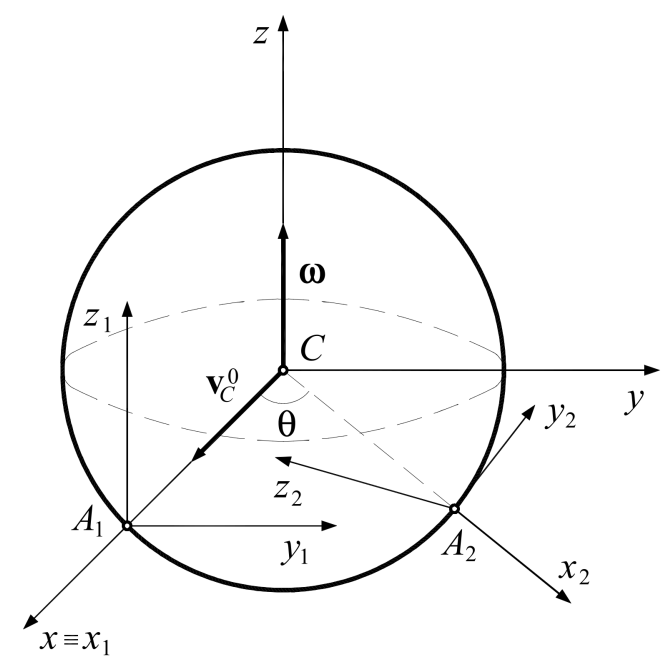

Figure 3. Example 1.

$$
\begin{aligned}
& {\left[\mathbf{D}_{1}\right]=\left[\mathbf{S}_{1}\right]\left[\mathbf{R}_{1}\right]=\left[\begin{array}{ccc}
0 & 0 & 0 \\
0 & 0 & -R \\
0 & R & 0
\end{array}\right],\left[\mathbf{D}_{2}\right]=\left[\mathbf{S}_{2}\right]\left[\mathbf{R}_{2}\right]=\left[\begin{array}{ccc}
0 & 0 & R \sin \theta \\
0 & 0 & -R \cos \theta \\
0 & R & 0
\end{array}\right]} \\
& \left\{\mathbf{v}_{C}^{0}\right\}=\left[\begin{array}{llllll}
0 & 0 & \omega_{0} & \omega_{0} R & 0 & 0
\end{array}\right]^{\mathrm{T}}, \\
& \left\{\mathbf{E}_{1 x}\right\}=\left[\begin{array}{llllll}
1 & 0 & 0 & 0 & 0 & 0
\end{array}\right]^{\mathrm{T}},\left\{\mathbf{E}_{2 x}\right\}=\left[\begin{array}{llllll}
\cos \theta & \sin \theta & 0 & 0 & 0 & 0
\end{array}\right]^{\mathrm{T}} \text {, }
\end{aligned}
$$




$$
\begin{aligned}
& {[\boldsymbol{\eta}]=\left[\begin{array}{llllll}
0 & 0 & 0 & 1 & 0 & 0 \\
0 & 0 & 0 & 0 & 1 & 0 \\
0 & 0 & 0 & 0 & 0 & 1 \\
1 & 0 & 0 & 0 & 0 & 0 \\
0 & 1 & 0 & 0 & 0 & 0 \\
0 & 0 & 1 & 0 & 0 & 0
\end{array}\right],} \\
& {[\mathbf{M}]=\left[\begin{array}{cccccc}
0 & 0 & 0 & m & 0 & 0 \\
0 & 0 & 0 & 0 & m & 0 \\
0 & 0 & 0 & 0 & 0 & m \\
J & 0 & 0 & 0 & 0 & 0 \\
0 & J & 0 & 0 & 0 & 0 \\
0 & 0 & J & 0 & 0 & 0
\end{array}\right],[\mathbf{M}]^{-1}=\left[\begin{array}{cccccc}
0 & 0 & 0 & J^{-1} & 0 & 0 \\
0 & 0 & 0 & 0 & J^{-1} & 0 \\
0 & 0 & 0 & 0 & 0 & J^{-1} \\
m^{-1} & 0 & 0 & 0 & 0 & 0 \\
0 & m^{-1} & 0 & 0 & 0 & 0 \\
0 & 0 & m^{-1} & 0 & 0 & 0
\end{array}\right],} \\
& g_{11 x x}=\left\{\mathbf{E}_{1 x}\right\}^{\mathrm{T}}[\boldsymbol{\eta}][\mathbf{M}]^{-1}\left\{\mathbf{E}_{1 x}\right\}=\frac{1}{m}, g_{22 x x}=\left\{\mathbf{E}_{2 x}\right\}^{\mathrm{T}}[\boldsymbol{\eta}][\mathbf{M}]^{-1}\left\{\mathbf{E}_{2 x}\right\}=\frac{1}{m}, \\
& g_{12 x x}=g_{21 x x}=\left\{\mathbf{E}_{1 x}\right\}^{\mathrm{T}}[\mathbf{\eta}][\mathbf{M}]^{-1}\left\{\mathbf{E}_{2 x}\right\}=\frac{\cos \theta}{m}, \\
& {\left[\begin{array}{ll}
g_{11 x x} & g_{12 x x} \\
g_{21 x x} & g_{22 x x}
\end{array}\right]=\left[\begin{array}{ll}
m^{-1} & \frac{\cos \theta}{m} \\
\frac{\cos \theta}{m} & m^{-1}
\end{array}\right]=\frac{\sin ^{2} \theta}{m^{2}},} \\
& \left\{\mathbf{E}_{1 y}\right\}=\left[\begin{array}{llllll}
0 & 1 & 0 & 0 & 0 & R
\end{array}\right]^{\mathrm{T}},\left\{\mathbf{E}_{2 y}\right\}=\left[\begin{array}{llllll}
-\sin \theta & \cos \theta & 0 & 0 & 0 & R
\end{array}\right]^{\mathrm{T}} \text {, }
\end{aligned}
$$

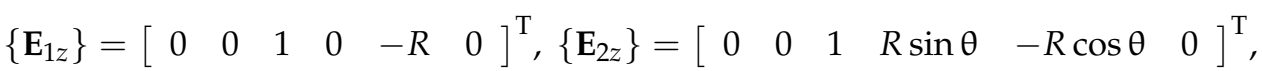

$$
\begin{aligned}
& v_{1 x}^{0}=\left\{\mathbf{E}_{1 x}\right\}^{\mathrm{T}}[\boldsymbol{\eta}]\left\{\mathbf{v}_{C}^{0}\right\}=\omega_{0} R, v_{1 y}^{0}=\left\{\mathbf{E}_{1 y}\right\}^{\mathrm{T}}[\boldsymbol{\eta}]\left\{\mathbf{v}_{C}^{0}\right\}=\omega_{0} R, v_{1 z}^{0}=\left\{\mathbf{E}_{1 z}\right\}^{\mathrm{T}}[\boldsymbol{\eta}]\left\{\mathbf{v}_{C}^{0}\right\}=0, \\
& v_{2 x}^{0}=\left\{\mathbf{E}_{2 x}\right\}^{\mathrm{T}}[\boldsymbol{\eta}]\left\{\mathbf{v}_{C}^{0}\right\}=\omega_{0} R \cos \theta, v_{2 y}^{0}=\left\{\mathbf{E}_{2 y}\right\}^{\mathrm{T}}[\boldsymbol{\eta}]\left\{\mathbf{v}_{C}^{0}\right\}=\omega_{0} R(1-\sin \theta), v_{2 z}^{0}=\left\{\mathbf{E}_{2 z}\right\}^{\mathrm{T}}[\boldsymbol{\eta}]\left\{\mathbf{v}_{\mathrm{C}}^{0}\right\}=0 .
\end{aligned}
$$

The Newton model yields

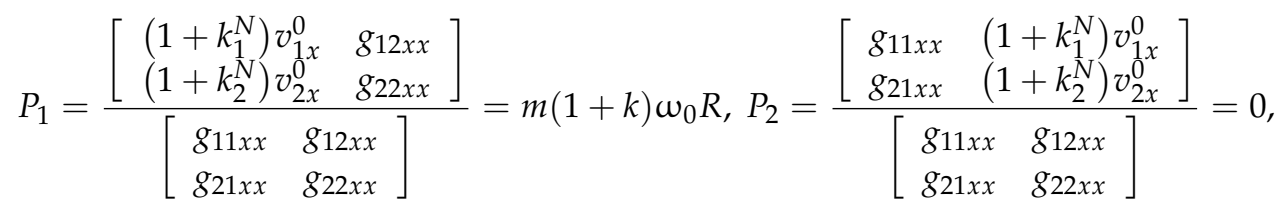

$$
\begin{aligned}
& \left\{\mathbf{v}_{C}\right\}=\left\{\mathbf{v}_{C}^{0}\right\}-P_{1}[\mathbf{M}]^{-1}\left\{\mathbf{E}_{1 x}\right\}-P_{2}[\mathbf{M}]^{-1}\left\{\mathbf{E}_{2 x}\right\}=\left[\begin{array}{llllll}
0 & 0 & \omega_{0} & -\omega_{0} R k & 0 & 0
\end{array}\right]^{\mathrm{T}} \text {, } \\
& g_{11 y x}=\left\{\mathbf{E}_{1 y}\right\}^{\mathrm{T}}[\mathbf{\eta}][\mathbf{M}]^{-1}\left\{\mathbf{E}_{1 x}\right\}=0, g_{12 y x}=\left\{\mathbf{E}_{1 y}\right\}^{\mathrm{T}}[\mathbf{\eta}][\mathbf{M}]^{-1}\left\{\mathbf{E}_{2 x}\right\}=\frac{\sin \theta}{m}, \\
& g_{11 z x}=\left\{\mathbf{E}_{1 z}\right\}^{\mathrm{T}}[\mathbf{\eta}][\mathbf{M}]^{-1}\left\{\mathbf{E}_{1 x}\right\}=0, g_{12 z x}=\left\{\mathbf{E}_{1 z}\right\}^{\mathrm{T}}[\mathbf{\eta}][\mathbf{M}]^{-1}\left\{\mathbf{E}_{2 x}\right\}=0, \\
& g_{21 y x}=\left\{\mathbf{E}_{2 y}\right\}^{\mathrm{T}}[\mathbf{\eta}][\mathbf{M}]^{-1}\left\{\mathbf{E}_{1 x}\right\}=-\frac{\sin \theta}{m}, g_{22 y x}=\left\{\mathbf{E}_{2 y}\right\}^{\mathrm{T}}[\boldsymbol{\eta}][\mathbf{M}]^{-1}\left\{\mathbf{E}_{2 x}\right\}=0, \\
& g_{21 z x}=\left\{\mathbf{E}_{2 z}\right\}^{\mathrm{T}}[\mathbf{\eta}][\mathbf{M}]^{-1}\left\{\mathbf{E}_{1 x}\right\}=0, g_{22 z x}=\left\{\mathbf{E}_{2 z}\right\}^{\mathrm{T}}[\mathbf{\eta}][\mathbf{M}]^{-1}\left\{\mathbf{E}_{2 x}\right\}=0, \\
& v_{1 y}=v_{1 y}^{0}-P_{1} g_{11 y x}-P_{2} g_{12 y x}=\omega_{0} R, v_{1 z}=v_{1 z}^{0}-P_{1} g_{11 z x}-P_{2} g_{12 z x}=0 \text {, } \\
& v_{2 y}=v_{2 y}^{0}-P_{1} g_{21 y x}-P_{2} g_{22 y x}=\omega_{0} R(1+k \sin \theta), v_{2 z}=v_{2 z}^{0}-P_{1} g_{21 z x}-P_{2} g_{22 z x}=0 \text {, } \\
& \cos \alpha_{1}^{0}=\frac{v_{1 y}^{0}}{\sqrt{\left(v_{1 y}^{0}\right)^{2}+\left(v_{1 z}^{0}\right)^{2}}}=1, \sin \alpha_{1}^{0}=\frac{v_{1 z}^{0}}{\sqrt{\left(v_{1 y}^{0}\right)^{2}+\left(v_{1 z}^{0}\right)^{2}}}=0, \alpha_{1}^{0}=0^{\circ}, \\
& \cos \alpha_{2}^{0}=\frac{v_{2 y}^{0}}{\sqrt{\left(v_{2 y}^{0}\right)^{2}+\left(v_{2 z}^{0}\right)^{2}}}=1, \sin \alpha_{2}^{0}=\frac{v_{2 z}^{0}}{\sqrt{\left(v_{2 y}^{0}\right)^{2}+\left(v_{2 z}^{0}\right)^{2}}}=0, \alpha_{2}^{0}=0^{\circ},
\end{aligned}
$$




$$
\begin{gathered}
\cos \alpha_{1}=\frac{v_{1 y}}{\sqrt{v_{1 y}^{2}+v_{1 z}^{2}}}=1, \sin \alpha_{1}=\frac{v_{1 z}}{\sqrt{v_{1 y}^{2}+v_{1 z}^{2}}}=0, \alpha_{1}=0^{\circ}, \\
\cos \alpha_{2}=\frac{v_{2 y}}{\sqrt{v_{2 y}^{2}+v_{2 z}^{2}}}=1, \sin \alpha_{2}=\frac{v_{2 z}}{\sqrt{v_{2 y}^{2}+v_{2 z}^{2}}}=0, \alpha_{2}=0^{\circ}, \\
E_{p}=\frac{1}{2}\left\{\left\{\mathbf{v}_{C}\right\}-\left\{\mathbf{v}_{C}^{0}\right\}\right\}^{\mathrm{T}}[\boldsymbol{\eta}][\mathbf{M}]\left\{\left\{\mathbf{v}_{C}\right\}-\left\{\mathbf{v}_{C}^{0}\right\}\right\}=\frac{1}{2} m \omega_{0}^{2} R^{2}(1+k)^{2}, \\
\Delta E_{c}=-E_{p}+P_{1} v_{1 x}^{0}+P_{2} v_{2 x}^{0}=\frac{1}{2} m \omega_{0}^{2} R^{2}\left(1-k^{2}\right) .
\end{gathered}
$$

For the Poisson model, one obtains

$$
\begin{gathered}
P_{1}^{c}=\frac{\left|\begin{array}{ll}
v_{1 x}^{0} & g_{12 x x} \\
v_{2 x}^{0} & g_{22 x x}
\end{array}\right|}{\left|\begin{array}{ll}
g_{11 x x} & g_{12 x x} \\
g_{21 x x} & g_{22 x x}
\end{array}\right|}=m \omega_{0} R, P_{2}^{c}=\frac{\left|\begin{array}{ll}
g_{11 x x} & v_{1 x}^{0} \\
g_{21 x x} & v_{2 x}^{0}
\end{array}\right|}{\left|\begin{array}{ll}
g_{11 x x} & g_{12 x x} \\
g_{21 x x} & g_{22 x x}
\end{array}\right|}=0, \\
P_{1}=P_{1}^{c}\left(1+k_{1}^{P}\right)=m \omega_{0}(1+k) R, P_{2}=P_{1}^{d}\left(1+k_{2}^{P}\right)=0 .
\end{gathered}
$$

The rest of results are identical to those obtained in the Newton case.

As numerical values, we consider $\omega_{0}=3 \mathrm{rad} / \mathrm{s}, R=0.25 \mathrm{~m}, k=0.5$, resulting in

$$
\begin{aligned}
& {[\boldsymbol{\eta}]=\left[\begin{array}{llllll}
0 & 0 & 0 & 1 & 0 & 0 \\
0 & 0 & 0 & 0 & 1 & 0 \\
0 & 0 & 0 & 0 & 0 & 1 \\
1 & 0 & 0 & 0 & 0 & 0 \\
0 & 1 & 0 & 0 & 0 & 0 \\
0 & 0 & 1 & 0 & 0 & 0
\end{array}\right]} \\
& {[\mathbf{M}]=\left[\begin{array}{cccccc}
0 & 0 & 0 & 10 & 0 & 0 \\
0 & 0 & 0 & 0 & 10 & 0 \\
0 & 0 & 0 & 0 & 0 & 10 \\
0.25 & 0 & 0 & 0 & 0 & 0 \\
0 & 0.25 & 0 & 0 & 0 & 0 \\
0 & 0 & 0.25 & 0 & 0 & 0
\end{array}\right],[\mathbf{M}]^{-1}=\left[\begin{array}{cccccc}
0 & 0 & 0 & 4 & 0 & 0 \\
0 & 0 & 0 & 0 & 4 & 0 \\
0 & 0 & 0 & 0 & 0 & 4 \\
0.1 & 0 & 0 & 0 & 0 & 0 \\
0 & 0.1 & 0 & 0 & 0 & 0 \\
0 & 0 & 0.1 & 0 & 0 & 0
\end{array}\right]} \\
& \left\{\mathbf{v}_{C}^{0}\right\}=\left[\begin{array}{llllll}
0 & 0 & 3 & 0.75 & 0 & 0
\end{array}\right]^{\mathrm{T}} \text {, } \\
& {\left[\mathbf{R}_{1}\right]=\left[\begin{array}{lll}
1 & 0 & 0 \\
0 & 1 & 0 \\
0 & 0 & 1
\end{array}\right],\left[\mathbf{R}_{2}\right]=\left[\begin{array}{ccc}
0.707107 & -0.707107 & 0 \\
0.707107 & 0.707107 & 0 \\
0 & 0 & 1
\end{array}\right] \text {, }} \\
& {\left[\mathbf{S}_{1}\right]=\left[\begin{array}{ccc}
0 & 0 & 0 \\
0 & 0 & -0.25 \\
0 & 0.25 & 0
\end{array}\right],\left[\mathbf{S}_{2}\right]=\left[\begin{array}{ccc}
0 & 0 & 0.176777 \\
0 & 0 & -0.176777 \\
-0.176777 & 0.176777 & 0
\end{array}\right] \text {, }} \\
& {\left[\mathbf{D}_{1}\right]=\left[\begin{array}{ccc}
0 & 0 & 0 \\
0 & 0 & -0.25 \\
0 & 0.25 & 0
\end{array}\right],\left[\mathbf{D}_{2}\right]=\left[\begin{array}{ccc}
0 & 0 & 0.176777 \\
0 & 0 & -0.176777 \\
0 & 0.25 & 0
\end{array}\right],} \\
& \left\{\mathbf{E}_{1 x}\right\}=\left[\begin{array}{llllll}
1 & 0 & 0 & 0 & 0 & 0
\end{array}\right]^{\mathrm{T}},\left\{\mathbf{E}_{2 x}\right\}=\left[\begin{array}{llllll}
0.707107 & 0.707107 & 0 & 0 & 0 & 0
\end{array}\right]^{\mathrm{T}} \text {, } \\
& \left\{\mathbf{E}_{1 y}\right\}=\left[\begin{array}{llllll}
0 & 1 & 0 & 0 & 0 & 0.25
\end{array}\right]^{\mathrm{T}},\left\{\mathbf{E}_{2 y}\right\}=\left[\begin{array}{llllll}
-0.707107 & 0,707107 & 0 & 0 & 0 & 0.25
\end{array}\right]^{\mathrm{T}} \text {, } \\
& \left\{\mathbf{E}_{1 z}\right\}=\left[\begin{array}{llllll}
0 & 0 & 1 & 0 & -0.25 & 0
\end{array}\right]^{\mathrm{T}},\left\{\mathbf{E}_{2 z}\right\}=\left[\begin{array}{llllll}
0 & 0 & 1 & 0.176777 & -0.176777 & 0
\end{array}\right]^{\mathrm{T}} \text {, } \\
& g_{11 x x}=0.1, g_{12 x x}=0.070711, g_{21 x x}=0.070711, g_{22 x x}=0.1 \text {, }
\end{aligned}
$$




$$
\begin{gathered}
g_{11 y x}=0, g_{12 y x}=0.070711, g_{11 z x}=0, g_{12 z x}=0, \\
g_{21 y x}=-0.070711, g_{22 y x}=0, g_{21 z x}=0, g_{22 z x}=0, \\
v_{1 x}^{0}=0.75, v_{1 y}^{0}=0.75, v_{1 z}^{0}=0, \\
v_{2 x}^{0}=0.538330, v_{2 y}^{0}=0.219670, v_{2 z}^{0}=0, \\
P_{1 N}=11.25, P_{2 N}=0, \\
\left\{\mathbf{v}_{C}\right\}=\left[\begin{array}{llll}
0 & 0 & 3 & -0.375 \quad 0 \quad 0
\end{array}\right], \\
v_{1 y}=0.75, v_{1 z}=0 \\
v_{2 y}=1.015165, v_{2 z}=0 \\
\cos \alpha_{1}^{0}=1, \sin \alpha_{1}^{0}=0, \alpha_{1}^{0}=0, \\
\cos \alpha_{2}^{0}=1, \sin \alpha_{2}^{0}=0, \alpha_{2}^{0}=0, \\
\cos \alpha_{1}=1, \sin \alpha_{1}=0, \alpha_{1}=0, \\
\cos \alpha_{2}=1, \sin \alpha_{2}=0, \alpha_{2}=1, \\
E_{p N}=6.328125, \Delta E_{c N}=2.109375, \\
P_{1 P}=11.25, P_{2 P}=0 .
\end{gathered}
$$

Example 2. The collision of an ellipsoid at two points (Figure 4a).

The equation of the ellipsoid is

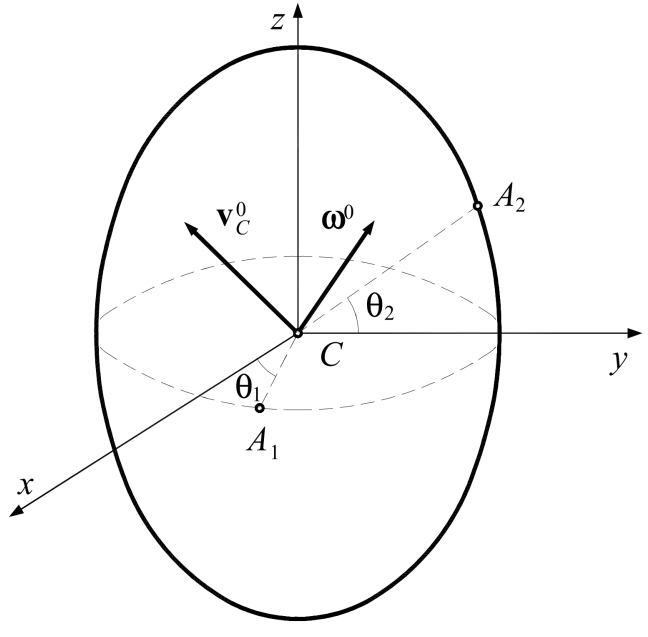

a)

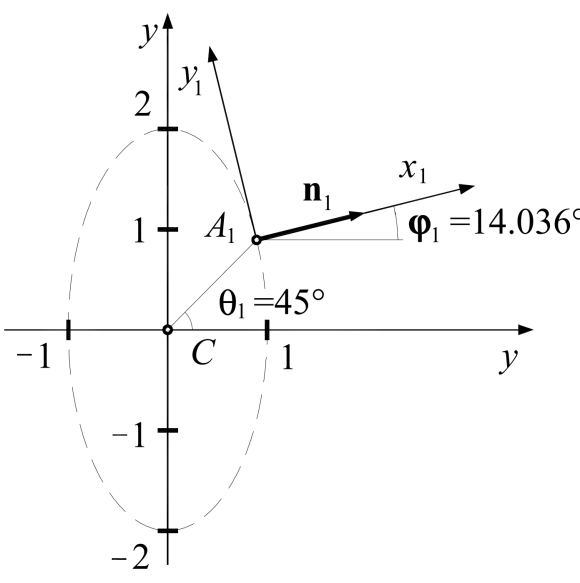

b)

Figure 4. Cont. 


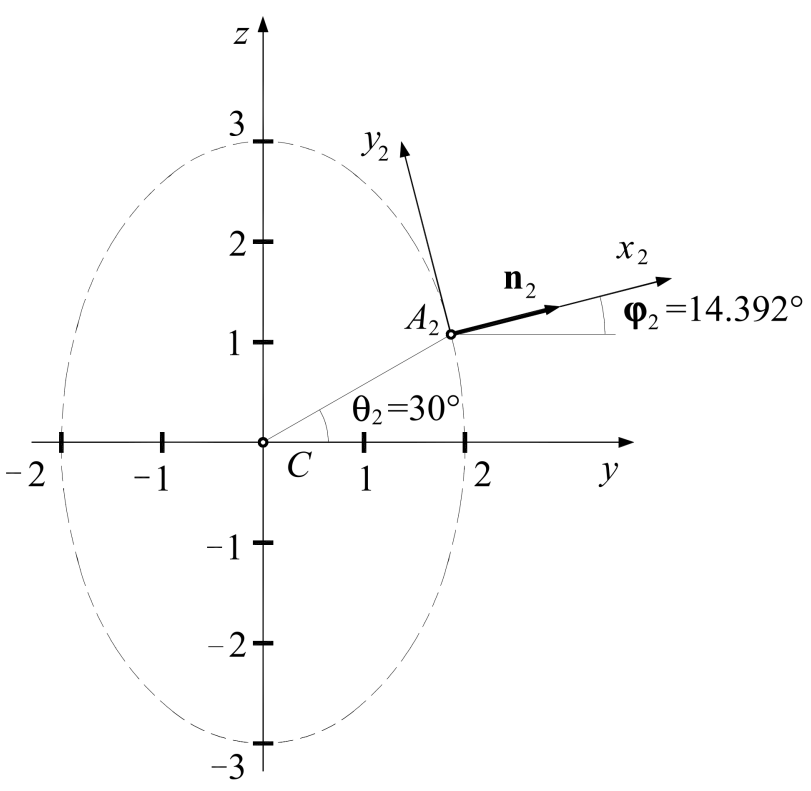

c)

Figure 4. Example 2. (a) Formulation of the problem; (b,c) some calculations.

$$
\frac{x^{2}}{1}+\frac{y^{2}}{4}+\frac{z^{2}}{9}=1
$$

One knows $\theta_{1}=45^{\circ}, \theta_{2}=30^{\circ}, \mathbf{v}_{C}^{0}=5 \mathbf{i}+7 \mathbf{j}+4 \mathbf{k}, \mathbf{\omega}^{0}=3 \mathbf{i}+\mathbf{j}+2 \mathbf{k}$.

The coordinates of point $A_{1}$ are obtained from the system

$$
\left\{\begin{array}{c}
x=y \\
\frac{x^{2}}{1}+\frac{y^{2}}{4}=1
\end{array},\right.
$$

wherefrom (Figure 4b) $x_{A_{1}}=\frac{2}{\sqrt{5}}, y_{A_{1}}=\frac{2}{\sqrt{5}}, z_{A_{1}}=0$.

Denoting the function $f(x, y, z)=\frac{x^{2}}{1}+\frac{y^{2}}{4}+\frac{z^{2}}{9}-1=0$, a vector which has the direction of the normal $\mathbf{n}_{1}$ is given by

$$
\left.\nabla f\right|_{\mathbf{A}_{1}}=\frac{2 x_{A_{1}}}{1} \mathbf{i}+\frac{2 y_{A_{1}}}{4} \mathbf{j}+0 \cdot \mathbf{k}=\frac{4}{\sqrt{5}} \mathbf{i}+\frac{1}{\sqrt{5}} \mathbf{j},
$$

wherefrom $\varphi_{1}=\frac{1}{4}, \varphi_{2}=14.03624347^{\circ}, \cos \varphi_{1}=\frac{4}{\sqrt{17}}, \sin \varphi_{1}=\frac{1}{\sqrt{17}}$.

Proceeding analogically for the point $A_{2}$, one obtains (Figure $4 \mathrm{c}$ )

$$
\begin{gathered}
\left\{\begin{array}{c}
z=\frac{\sqrt{3}}{3} y \\
y^{2}+\frac{z^{2}}{9}=1
\end{array}\right. \\
y_{A_{2}}=6 \sqrt{\frac{3}{31}}, z_{A_{2}}=\frac{6}{\sqrt{31}}, x_{A_{2}}=0, \\
\left.\nabla f\right|_{\mathbf{A}_{2}}=0 \cdot \mathbf{i}+\frac{2 y_{A_{2}} \mathbf{j}+\frac{2 z_{A_{2}}}{9} \mathbf{k}=\frac{3 \sqrt{3}}{\sqrt{311}} \mathbf{j}+\frac{4}{3 \sqrt{31}} \mathbf{k},}{\varphi_{2}=\frac{4}{9 \sqrt{3}}, \varphi_{2}=14.3916014, \cos \varphi_{2}=\frac{9 \sqrt{3}}{\sqrt{259}}, \sin \varphi_{2}=\frac{4}{\sqrt{259}} .} .
\end{gathered}
$$


The rotational matrices read

$$
\begin{gathered}
{\left[\mathbf{R}_{1}\right]=\left[\begin{array}{ccc}
1 & 0 & 0 \\
0 & \cos \varphi_{1} & -\sin \varphi_{1} \\
0 & \sin \varphi_{1} & \cos \varphi_{1}
\end{array}\right]=\left[\begin{array}{ccc}
1 & 0 & 0 \\
0 & \frac{4}{\sqrt{17}} & -\frac{1}{\sqrt{17}} \\
0 & \frac{1}{\sqrt{17}} & \frac{4}{\sqrt{17}}
\end{array}\right],} \\
{\left[\mathbf{R}_{2}\right]=\left[\begin{array}{cccc}
0 & -1 & 0 \\
1 & 0 & 0 \\
0 & 0 & 1
\end{array}\right]\left[\begin{array}{ccc}
\cos \left(-\varphi_{2}\right) & 0 & \sin \left(-\varphi_{2}\right) \\
0 & 1 & 0 \\
-\sin \left(-\varphi_{2}\right) & 0 & \cos \varphi_{2}
\end{array}\right]=\left[\begin{array}{ccc}
0 & -1 & 0 \\
\frac{9 \sqrt{3}}{\sqrt{259}} & 0 & -\frac{4}{\sqrt{259}} \\
\frac{4}{\sqrt{259}} & 0 & \frac{9 \sqrt{3}}{\sqrt{259}}
\end{array}\right] .}
\end{gathered}
$$

For the coefficients of restitution, we choose the following values:

- $\quad k_{1}^{N}=k_{1}^{P}=1.0, k_{2}^{N}=k_{2}^{P}=1.0($ case 1$) ;$

- $\quad k_{1}^{N}=k_{1}^{P}=0.0, k_{2}^{N}=k_{2}^{P}=0.0$ (case 2);

- $\quad k_{1}^{N}=k_{1}^{P}=1.0, k_{2}^{N}=k_{2}^{P}=0.0($ case 3);

- $\quad k_{1}^{N}=k_{1}^{P}=0.5, k_{2}^{N}=k_{2}^{P}=0.5($ case 4$) ;$

- $\quad k_{1}^{N}=k_{1}^{P}=0.7, k_{2}^{N}=k_{2}^{P}=0.3$ (case 5).

The results are presented in Table 1.

Table 1. Results for the numerical cases considered in Example 2.

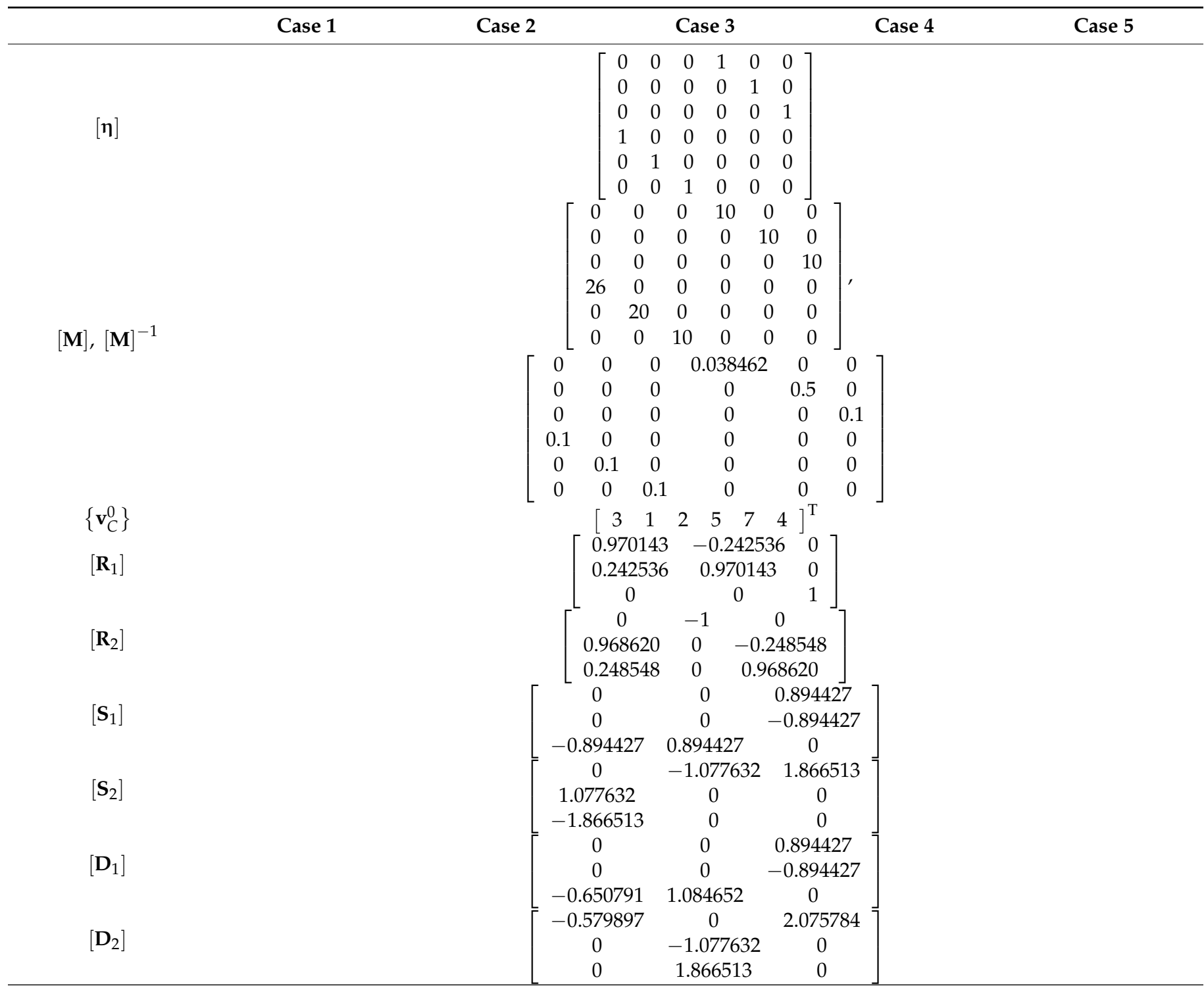


Table 1. Cont.

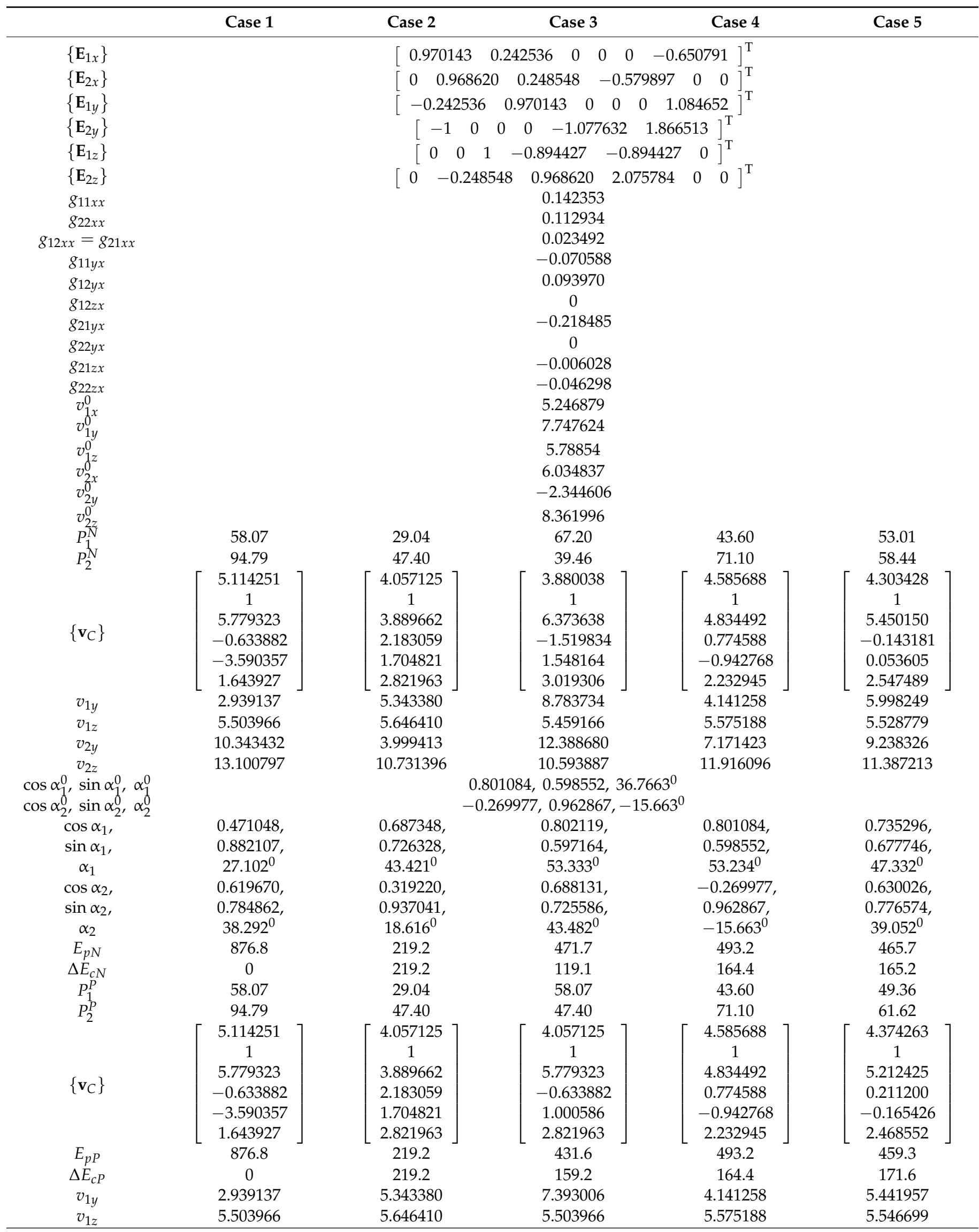


Table 1. Cont.

\begin{tabular}{cccccc}
\hline & Case 1 & Case 2 & Case 3 & Case 4 & Case 5 \\
\hline$v_{2 y}$ & 10.343432 & 3.999413 & 10.343432 & 7.171423 & 8.440226 \\
$v_{2 z}$ & 13.100797 & 10.731396 & 10.593887 & 11.916096 & 11.387213 \\
\hline
\end{tabular}

The values obtained depend on which coefficient of restitution is used (Newton or Poisson). The same values result only if the coefficients of restitution at the points are equal (no matter their common value). This result is new, and it was not reported previously in the references.

Example 3. Planar collision of a bar at two points (Figure 5).

One knows: $A B=2 A C=2 C B=6 l, l_{1}=\frac{l}{2}, l_{2}=l, k_{1}^{N}=k_{1}^{P}=k_{1}=0.3, k_{2}^{N}=$ $k_{2}^{P}=k_{2}=0.2, \mathbf{v}_{C}^{0}=3 \mathbf{i}+2 \mathbf{j}, \boldsymbol{\omega}^{0}=0.4, l=4 \mathrm{~m}, m=1 \mathrm{~kg}, J_{x}=J_{z}=J_{1}=\frac{3}{4} m l^{2}$, and $J_{y}=J_{2}=0.1 m l^{2}$.

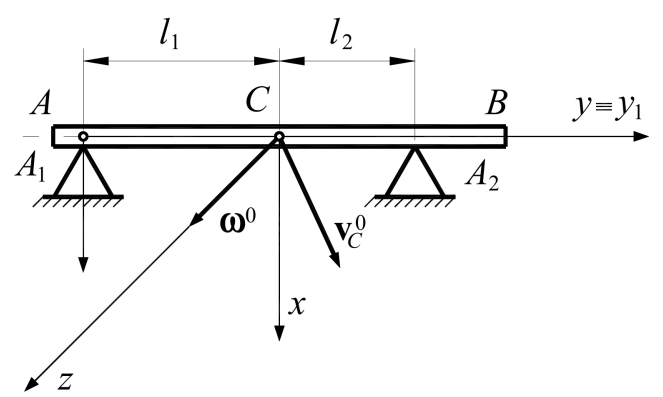

Figure 5. Example 3.

Proceeding analogically, one obtains:

- $\quad$ for the Newton model:

$$
\begin{gathered}
x_{A_{1}}=0, y_{A_{1}}=-l_{1}=-\frac{l}{2}, z_{A_{1}}=0, \\
x_{A_{2}}=0, y_{A_{2}}=l_{2}=l, z_{A_{2}}=0, \\
{\left[\mathbf{R}_{1}\right]=\left[\mathbf{R}_{2}\right]=[\mathbf{I}]=\left[\begin{array}{ccc}
1 & 0 & 0 \\
0 & 1 & 0 \\
0 & 0 & 1
\end{array}\right],} \\
{\left[\mathbf{S}_{1}\right]=\left[\begin{array}{ccc}
0 & -z_{A_{1}} & y_{A_{1}} \\
z_{A_{1}} & 0 & -x_{A_{1}} \\
-y_{A_{1}} & x_{A_{1}} & 0
\end{array}\right]=\left[\begin{array}{ccc}
0 & 0 & -\frac{l}{2} \\
0 & 0 & 0 \\
\frac{l}{2} & 0 & 0
\end{array}\right]=\left[\begin{array}{ccc}
0 & 0 & -2 \\
0 & 0 & 0 \\
2 & 0 & 0
\end{array}\right],} \\
\left.-\mathbf{S}_{2}\right]=\left[\begin{array}{ccc}
0 & 0 & l \\
z_{A_{2}} & 0 & -x_{A_{2}} \\
-y_{A_{2}} & x_{A_{2}} & 0
\end{array}\right]=\left[\begin{array}{ccc}
0 & 0 & 0 \\
-l & 0 & 0
\end{array}\right]=\left[\begin{array}{ccc}
0 & 0 & 4 \\
0 & 0 & 0 \\
-4 & 0 & 0
\end{array}\right],
\end{gathered}
$$$$
\left[\mathbf{D}_{1}\right]=\left[\mathbf{S}_{1}\right]\left[\mathbf{R}_{1}\right]=\left[\begin{array}{ccc}
0 & 0 & -\frac{l}{2} \\
0 & 0 & 0 \\
\frac{l}{2} & 0 & 0
\end{array}\right]=\left[\begin{array}{ccc}
0 & 0 & -2 \\
0 & 0 & 0 \\
2 & 0 & 0
\end{array}\right],\left[\mathbf{D}_{2}\right]=\left[\mathbf{S}_{2}\right]\left[\mathbf{R}_{2}\right]=\left[\begin{array}{ccc}
0 & 0 & l \\
0 & 0 & 0 \\
-l & 0 & 0
\end{array}\right]=\left[\begin{array}{ccc}
0 & 0 & 4 \\
0 & 0 & 0 \\
-4 & 0 & 0
\end{array}\right],
$$ 


$$
\begin{aligned}
& {[\boldsymbol{\eta}]=\left[\begin{array}{llllll}
0 & 0 & 0 & 1 & 0 & 0 \\
0 & 0 & 0 & 0 & 1 & 0 \\
0 & 0 & 0 & 0 & 0 & 1 \\
1 & 0 & 0 & 0 & 0 & 0 \\
0 & 1 & 0 & 0 & 0 & 0 \\
0 & 0 & 1 & 0 & 0 & 0
\end{array}\right]} \\
& {[\mathbf{M}]=\left[\begin{array}{cccccc}
0 & 0 & 0 & m & 0 & 0 \\
0 & 0 & 0 & 0 & m & 0 \\
0 & 0 & 0 & 0 & 0 & m \\
J_{1} & 0 & 0 & 0 & 0 & 0 \\
0 & J_{2} & 0 & 0 & 0 & 0 \\
0 & 0 & J_{1} & 0 & 0 & 0
\end{array}\right]=\left[\begin{array}{cccccc}
0 & 0 & 0 & 1 & 0 & 0 \\
0 & 0 & 0 & 0 & 1 & 0 \\
0 & 0 & 0 & 0 & 0 & 1 \\
12 & 0 & 0 & 0 & 0 & 0 \\
0 & 1.6 & 0 & 0 & 0 & 0 \\
0 & 0 & 12 & 0 & 0 & 0
\end{array}\right]} \\
& {[\mathbf{M}]^{-1}=\left[\begin{array}{cccccc}
0 & 0 & 0 & J_{1}^{-1} & 0 & 0 \\
0 & 0 & 0 & 0 & J_{1}^{-1} & 0 \\
0 & 0 & 0 & 0 & 0 & J_{1}^{-1} \\
m^{-1} & 0 & 0 & 0 & 0 & 0 \\
0 & m^{-1} & 0 & 0 & 0 & 0 \\
0 & 0 & m^{-1} & 0 & 0 & 0
\end{array}\right]=\left[\begin{array}{cccccc}
0 & 0 & 0 & 0.083333 & 0 & 0 \\
0 & 0 & 0 & 0 & 0.625 & 0 \\
0 & 0 & 0 & 0 & 0 & 0.083333 \\
1 & 0 & 0 & 0 & 0 & 0 \\
0 & 1 & 0 & 0 & 0 & 0 \\
0 & 0 & 1 & 0 & 0 & 0
\end{array}\right]} \\
& \left\{\mathbf{v}_{C}^{0}\right\}=\left[\begin{array}{llllll}
0 & 0 & \omega_{z}^{0} & v_{x}^{0} & v_{y}^{0} & 0
\end{array}\right]^{\mathrm{T}}=\left[\begin{array}{llllll}
0 & 0 & 0.4 & 3 & 2 & 0
\end{array}\right]^{\mathrm{T}}, \\
& \left\{\mathbf{E}_{1 x}\right\}=\left[\begin{array}{llllll}
1 & 0 & 0 & 0 & 0 & \frac{l}{2}
\end{array}\right]^{\mathrm{T}}=\left[\begin{array}{llllll}
1 & 0 & 0 & 0 & 0 & 2
\end{array}\right]^{\mathrm{T}},\left\{\mathbf{E}_{1 y}\right\}=\left[\begin{array}{llllll}
0 & 1 & 0 & 0 & 0 & 0
\end{array}\right]^{\mathrm{T}}, \\
& \left\{\mathbf{E}_{1 z}\right\}=\left[\begin{array}{llllll}
0 & 0 & 1 & -\frac{l}{2} & 0 & 0
\end{array}\right]^{\mathrm{T}}=\left[\begin{array}{llllll}
0 & 0 & 1 & -2 & 0 & 0
\end{array}\right]^{\mathrm{T}} \text {, } \\
& \left\{\mathbf{E}_{2 z}\right\}=\left[\begin{array}{llllll}
0 & 0 & 1 & l & 0 & 0
\end{array}\right]^{\mathrm{T}}=\left[\begin{array}{llllll}
0 & 0 & 1 & 4 & 0 & 0
\end{array}\right]^{\mathrm{T}}, \\
& \begin{aligned}
& g_{11 x x}= \frac{1}{m}+\frac{l^{2}}{4 J_{1}}=1.333333, g_{11 y x}=0, g_{12 y x}=0, g_{21 x x}=\frac{1}{m}-\frac{l^{2}}{2 J_{1}}=0.333333, \\
& g_{11 z x}=0, g_{12 z x}=0, g_{21 y x}=0, g_{22 y x}=0, g_{21 z x}=0, g_{22 z x}=0,
\end{aligned}
\end{aligned}
$$$$
\left\{\mathbf{E}_{2 x}\right\}=\left[\begin{array}{llllll}
1 & 0 & 0 & 0 & 0 & -l
\end{array}\right]^{\mathrm{T}}=\left[\begin{array}{llllll}
1 & 0 & 0 & 0 & 0 & -4
\end{array}\right]^{\mathrm{T}},\left\{\mathbf{E}_{2 y}\right\}=\left[\begin{array}{llllll}
0 & 1 & 0 & 0 & 0 & 0
\end{array}\right]^{\mathrm{T}} \text {, }
$$$$
v_{1 x}^{0}=\left\{\mathbf{E}_{1 x}\right\}^{\mathrm{T}}[\boldsymbol{\eta}]\left\{\mathbf{v}_{C}^{0}\right\}=\boldsymbol{\omega}_{z}^{0} \frac{l}{2}+v_{x}^{0}=0.4 \times \frac{4}{2}+3=3.8, v_{1 y}^{0}=\left\{\mathbf{E}_{1 y}\right\}^{\mathrm{T}}[\boldsymbol{\eta}]\left\{\mathbf{v}_{C}^{0}\right\}=v_{y}^{0}=2, v_{1 z}^{0}=\left\{\mathbf{E}_{1 z}\right\}^{\mathrm{T}}[\boldsymbol{\eta}]\left\{\mathbf{v}_{C}^{0}\right\}=0
$$$$
v_{2 x}^{0}=\left\{\mathbf{E}_{2 x}\right\}^{\mathrm{T}}[\boldsymbol{\eta}]\left\{\mathbf{v}_{C}^{0}\right\}=-\omega_{z}^{0} l+v_{x}^{0}=-0.4 \times 4+3=1.4, v_{2 y}^{0}=\left\{\mathbf{E}_{2 y}\right\}^{\mathrm{T}}[\boldsymbol{\eta}]\left\{\mathbf{v}_{C}^{0}\right\}=v_{y}^{0}=2, v_{21 z}^{0}=\left\{\mathbf{E}_{2 z}\right\}^{\mathrm{T}}[\mathbf{\eta}]\left\{\mathbf{v}_{C}^{0}\right\}=0,
$$$$
P_{1}^{N}=\frac{\left|\begin{array}{cc}
\left(1+k_{1}^{N}\right) v_{1 x}^{0} & g_{12 x x} \\
\left(1+k_{2}^{N}\right) v_{2 x}^{0} & g_{22 x x}
\end{array}\right|}{\left|\begin{array}{ll}
g_{11 x x} & g_{12 x x} \\
g_{21 x x} & g_{22 x x}
\end{array}\right|}=3.655556, P_{2}^{N}=\frac{\left|\begin{array}{ll}
g_{11 x x} & \left(1+k_{1}^{N}\right) v_{1 x}^{0} \\
g_{21 x x} & \left(1+k_{2}^{N}\right) v_{2 x}^{0}
\end{array}\right|}{\left|\begin{array}{ll}
g_{11 x x} & g_{12 x x} \\
g_{21 x x} & g_{22 x x}
\end{array}\right|}=0.197778
$$

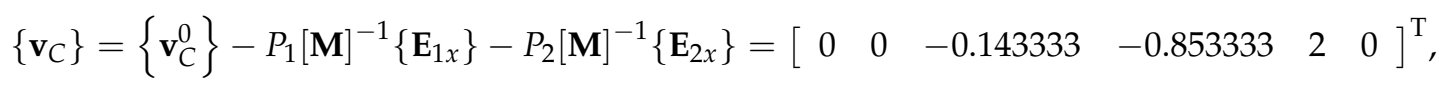$$
v_{1 y}=2, v_{1 z}=0, v_{2 y}=2, v_{2 z}=0,
$$$$
\cos \alpha_{1}^{0}=1, \sin \alpha_{1}^{0}=0, \alpha_{1}^{0}=0,
$$$$
\cos \alpha_{1}=1, \sin \alpha_{1}=0, \alpha_{1}=0,
$$$$
\cos \alpha_{2}^{0}=1, \sin \alpha_{2}^{0}=0, \alpha_{2}^{0}=0 \text {, }
$$ 


$$
\begin{gathered}
\cos \alpha_{2}=1, \sin \alpha_{2}=0, \alpha_{2}=0, \\
E_{p N}=9.195356, \Delta E_{c N}=4.972644, \\
P_{1 P}=3.64, P_{2 P}=0.24 ;
\end{gathered}
$$

- $\quad$ for the Poisson model:

$$
\begin{aligned}
& \left\{\mathbf{v}_{C}\right\}=\left[\begin{array}{llllll}
0 & 0 & -0.126667 & -0.88 & 2 & 0
\end{array}\right]^{\mathrm{T}} \text {, } \\
& v_{1 y}=2, v_{1 z}=0, v_{2 y}=2, v_{2 z}=0, \\
& \cos \alpha_{1}^{0}=1, \sin \alpha_{1}^{0}=0, \alpha_{1}^{0}=0, \\
& \cos \alpha_{1}=1, \sin \alpha_{1}=0, \alpha_{1}=0 \text {, } \\
& \cos \alpha_{2}^{0}=1, \sin \alpha_{2}^{0}=0, \alpha_{2}^{0}=0 \text {, } \\
& \cos \alpha_{2}=1, \sin \alpha_{2}=0, \alpha_{2}=0 \text {, } \\
& E_{c P}=9.191467, \Delta E_{c P}=4.976533 \text {. }
\end{aligned}
$$

A classical subcase is that defined by $l_{1}=l_{2}=l, k_{1}=k_{2}=k$, and $\boldsymbol{\omega}^{0}=0$. Assuming now that $k=0.1$, and $k=1.0$, with the rest of the values remaining unchanged, the following results are obtained

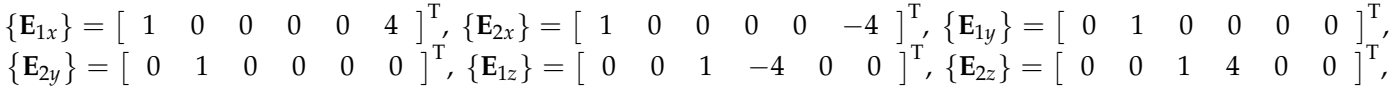

$$
\begin{aligned}
& g_{11 x x}=2.333333, g_{22 x x}=2.333333, g_{12 x x}=-0.333333, g_{21 x x}=-0.333333 \text {, } \\
& g_{11 y x}=0, g_{12 y x}=0, g_{11 z x}=0, g_{12 z x}=0, g_{21 y x}=0, g_{22 y x}=0, g_{21 z x}=0, g_{22 z x}=0 \text {, } \\
& v_{1 x}^{0}=3, v_{1 y}^{0}=2, v_{1 z}=0, v_{2 x}^{0}=3, v_{2 y}^{0}=2, v_{2 z}^{0}=0 ;
\end{aligned}
$$

- $\quad$ for $k=0.0$ (regardless of the model (Newton or Poisson) used):

$$
\begin{aligned}
& P_{1}^{N}=P_{1}^{P}=1.5, P_{2}^{N}=P_{2}^{P}=1.5, \\
& \left\{\mathbf{v}_{C}\right\}=\left[\begin{array}{llllll}
0 & 0 & 0 & 0 & 2 & 0
\end{array}\right]^{\mathrm{T}}, \\
& v_{1 y}=2, v_{1 z}=0, v_{2 y}=2, v_{2 z}=0 \text {, } \\
& \cos \alpha_{1}^{0}=1, \sin \alpha_{1}^{0}=0, \alpha_{1}^{0}=0, \\
& \cos \alpha_{2}^{0}=1, \sin \alpha_{2}^{0}=0, \alpha_{2}^{0}=0 \text {, } \\
& \cos \alpha_{1}=1, \sin \alpha_{1}=0, \alpha_{1}=0 \text {, } \\
& \cos \alpha_{2}=1, \sin \alpha_{2}=0, \alpha_{2}=0 \text {, } \\
& E_{p N}=E_{p P}=4.5, \Delta E_{c N}=\Delta E_{c P}=4.5 \text {; }
\end{aligned}
$$

- $\quad$ for $k=0.5$ (for both models (Newton and Poisson)):

$$
\begin{aligned}
& P_{1}^{N}=P_{1}^{P}=2.25, P_{2}^{N}=P_{2}^{P}=2.25, \\
& \left\{\mathbf{v}_{C}\right\}=\left[\begin{array}{llllll}
0 & 0 & -1.5 & 2 & 0 & 0
\end{array}\right]^{\mathrm{T}} \text {, } \\
& v_{1 y}=2, v_{1 z}=0, v_{2 y}=2, v_{2 z}=0 \text {, } \\
& \cos \alpha_{1}^{0}=1, \sin \alpha_{1}^{0}=0, \alpha_{1}^{0}=0, \\
& \cos \alpha_{2}^{0}=1, \sin \alpha_{2}^{0}=0, \alpha_{2}^{0}=0 \text {, } \\
& \cos \alpha_{1}=1, \sin \alpha_{1}=0, \alpha_{1}=0 \text {, }
\end{aligned}
$$




$$
\begin{gathered}
\cos \alpha_{2}=1, \sin \alpha_{2}=0, \alpha_{2}=0, \\
E_{p N}=E_{p P}=10.125, \Delta E_{c N}=\Delta E_{c P}=3.375 ;
\end{gathered}
$$

- $\quad$ for $k=1.0$ (both models):

$$
\begin{aligned}
& P_{1}^{N}=P_{1}^{P}=3, P_{2}^{N}=P_{2}^{P}=3, \\
& \left\{\mathbf{v}_{C}\right\}=\left[\begin{array}{llllll}
0 & 0 & 0 & -3 & 2 & 0
\end{array}\right]^{\mathrm{T}} \text {, } \\
& v_{1 y}=2, v_{1 z}=0, v_{2 y}=2, v_{2 z}=0 \text {, } \\
& \cos \alpha_{1}^{0}=1, \sin \alpha_{1}^{0}=0, \alpha_{1}^{0}=0, \\
& \cos \alpha_{2}^{0}=1, \sin \alpha_{2}^{0}=0, \alpha_{2}^{0}=0 \text {, } \\
& \cos \alpha_{1}=1, \sin \alpha_{1}=0, \alpha_{1}=0 \text {, } \\
& \cos \alpha_{2}=1, \sin \alpha_{2}=0, \alpha_{2}=0, \\
& E_{p N}=E_{p P}=18, \Delta E_{c N}=\Delta E_{c P}=0 \text {. }
\end{aligned}
$$

One may observe that the results are identical, regardless of which coefficient of restitution is used. This is a particular case in which both coefficients of restitution at the two points are equal.

Example 4. Spatial collision of a bar at two points (Figure 6).

One knows:

- $\quad$ the rotational matrix from the system OXYZ to the system $C x y z$,

$$
[\mathbf{A}]=[\boldsymbol{\psi}][\boldsymbol{\theta}][\boldsymbol{\varphi}],
$$

where $\psi=-45^{\circ}, \theta=0^{\circ}, \boldsymbol{\varphi}=-45^{\circ}$,

$$
[\boldsymbol{\psi}]=\left[\begin{array}{ccc}
1 & 0 & 0 \\
0 & \cos \psi & -\sin \psi \\
0 & \sin \psi & \cos \psi
\end{array}\right],[\boldsymbol{\theta}]=\left[\begin{array}{ccc}
\cos \theta & 0 & \sin \theta \\
0 & 1 & 0 \\
-\sin \theta & 0 & \cos \theta
\end{array}\right],[\boldsymbol{\varphi}]=\left[\begin{array}{ccc}
\cos \varphi & -\sin \varphi & 0 \\
\sin \varphi & \cos \varphi & 0 \\
0 & 0 & 1
\end{array}\right] ;
$$

- $\quad$ the mass of the bar $m=1 \mathrm{~kg}$;

- $\quad$ the dimensions $A_{1} C=C A_{2}=3 l=3 \mathrm{~m}$;

- $\quad$ the central principal moments of inertia $J_{x}=J_{z}=\frac{3}{4} m l^{2}=0.75 \mathrm{~kg} \mathrm{~m}^{2}$, and $J_{y}=$ $0.1 m l^{2}=0.1 \mathrm{~kg} \mathrm{~m}^{2}$;

- $\quad$ the initial angular velocity $\boldsymbol{\omega}^{0}=\omega_{x}^{0} \mathbf{i}+\omega_{y}^{0} \mathbf{j}+\omega_{z}^{0} \mathbf{k}$, where $\omega_{x}^{0}=1 \mathrm{rad} / \mathrm{s}, \omega_{y}^{0}=$ $2 \mathrm{rad} / \mathrm{s}$, and $\omega_{z}^{0}=0 \mathrm{rad} / \mathrm{s}$;

- $\quad$ the initial velocity of the center of mass $C, \mathbf{v}_{C}^{0}=v_{C x} \mathbf{i}+v_{C y} \mathbf{j}+v_{C z} \mathbf{k}$, with $v_{C x}^{0}=5 \mathrm{~m} / \mathrm{s}$, $v_{C y}^{0}=10 \mathrm{~m} / \mathrm{s}$, and $v_{C z}^{0}=2 \mathrm{~m} / \mathrm{s}$;

- the coefficients of restitution:

(i) $\quad k_{1}^{N}=k_{1}^{P}=1, k_{2}^{N}=k_{2}^{P}=1$ (case 1),

(ii) $\quad k_{1}^{N}=k_{1}^{P}=0.6, k_{2}^{N}=k_{2}^{P}=0.3$ (case 2), and

(iii) $\quad k_{1}^{N}=k_{1}^{P}=0, k_{2}^{N}=k_{2}^{P}=0$ (case 3). 


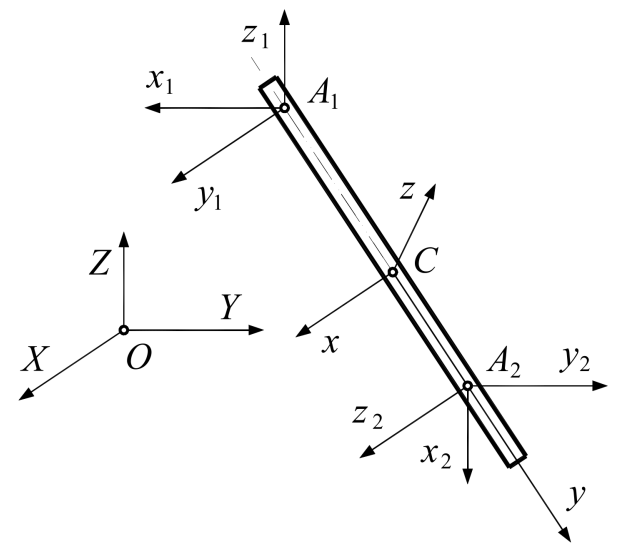

Figure 6. Example 4.

It results in the following

$$
\begin{aligned}
& {[\boldsymbol{\eta}]=\left[\begin{array}{llllll}
0 & 0 & 0 & 1 & 0 & 0 \\
0 & 0 & 0 & 0 & 1 & 0 \\
0 & 0 & 0 & 0 & 0 & 1 \\
1 & 0 & 0 & 0 & 0 & 0 \\
0 & 1 & 0 & 0 & 0 & 0 \\
0 & 0 & 1 & 0 & 0 & 0
\end{array}\right]} \\
& {[\mathbf{M}]=\left[\begin{array}{cccccc}
0 & 0 & 0 & 1 & 0 & 0 \\
0 & 0 & 0 & 0 & 1 & 0 \\
0 & 0 & 0 & 0 & 0 & 1 \\
0.75 & 0 & 0 & 0 & 0 & 0 \\
0 & 0.1 & 0 & 0 & 0 & 0 \\
0 & 0 & 0.75 & 0 & 0 & 0
\end{array}\right],[\mathbf{M}]^{-1}=\left[\begin{array}{cccccc}
0 & 0 & 0 & 1.333333 & 0 & 0 \\
0 & 0 & 0 & 0 & 10 & 0 \\
0 & 0 & 0 & 0 & 0 & 1.333333 \\
1 & 0 & 0 & 0 & 0 & 0 \\
0 & 1 & 0 & 0 & 0 & 0 \\
0 & 0 & 1 & 0 & 0 & 0
\end{array}\right],} \\
& \left\{\mathbf{v}_{C}^{0}\right\}=\left[\begin{array}{llllll}
1 & 2 & 0 & 5 & 10 & 2
\end{array}\right]^{\mathrm{T}}, \\
& {[\boldsymbol{\psi}]=\left[\begin{array}{ccc}
1 & 0 & 0 \\
0 & \frac{\sqrt{2}}{2} & \frac{\sqrt{2}}{2} \\
0 & -\frac{\sqrt{2}}{2} & \frac{\sqrt{2}}{2}
\end{array}\right],[\boldsymbol{\theta}]=\left[\begin{array}{lll}
1 & 0 & 0 \\
0 & 1 & 0 \\
0 & 0 & 1
\end{array}\right],[\boldsymbol{\varphi}]=\left[\begin{array}{ccc}
\frac{\sqrt{2}}{2} & \frac{\sqrt{2}}{2} & 0 \\
-\frac{\sqrt{2}}{2} & \frac{\sqrt{2}}{2} & 0 \\
0 & 0 & 1
\end{array}\right],} \\
& {[\mathbf{A}]=[\boldsymbol{\psi}][\boldsymbol{\theta}][\boldsymbol{\varphi}]=\left[\begin{array}{ccc}
\frac{\sqrt{2}}{2} & \frac{\sqrt{2}}{2} & 0 \\
-\frac{1}{2} & \frac{1}{2} & \frac{\sqrt{2}}{2} \\
0 & 0 & 1
\end{array}\right] \text {. }}
\end{aligned}
$$

The rotational matrices of the reference systems $A_{1} x_{1} y_{1} z_{1}$ and $A_{2} x_{2} y_{2} z_{2}$ relative to the reference system $C x y z$ read

$$
\left[\mathbf{A}_{1}\right]=\left[\begin{array}{ccc}
0 & -1 & 0 \\
1 & 0 & 0 \\
0 & 0 & 1
\end{array}\right],\left[\mathbf{A}_{2}\right]=\left[\begin{array}{ccc}
0 & 0 & 1 \\
0 & 1 & 0 \\
-1 & 0 & 0
\end{array}\right]
$$

wherefrom

$$
\left[\mathbf{R}_{1}\right]=[\mathbf{A}]^{-1}\left[\mathbf{A}_{1}\right]=\left[\begin{array}{ccc}
-\frac{1}{2} & -\frac{\sqrt{2}}{2} & \frac{1}{2} \\
\frac{1}{2} & -\frac{\sqrt{2}}{2} & -\frac{1}{2} \\
\frac{\sqrt{2}}{2} & 0 & \frac{\sqrt{2}}{2}
\end{array}\right],\left[\mathbf{R}_{2}\right]=[\mathbf{A}]^{-1}\left[\mathbf{A}_{2}\right]=\left[\begin{array}{ccc}
-\frac{1}{2} & -\frac{1}{2} & \frac{\sqrt{2}}{2} \\
\frac{1}{2} & \frac{1}{2} & \frac{\sqrt{2}}{2} \\
-\frac{\sqrt{2}}{2} & \frac{\sqrt{2}}{2} & 0
\end{array}\right] .
$$


We impose $X_{C}=0$ and from the relations

$$
\left[\begin{array}{l}
X_{A_{1}} \\
Y_{A_{1}} \\
Z_{A_{1}}
\end{array}\right]=\left[\begin{array}{c}
X_{C} \\
Y_{C} \\
Z_{C}
\end{array}\right]+[\mathbf{A}]\left[\begin{array}{c}
0 \\
-3 l \\
0
\end{array}\right],\left[\begin{array}{l}
X_{A_{2}} \\
Y_{A_{2}} \\
Z_{A_{2}}
\end{array}\right]=\left[\begin{array}{c}
X_{C} \\
Y_{C} \\
Z_{C}
\end{array}\right]+[\mathbf{A}]\left[\begin{array}{c}
0 \\
3 l \\
0
\end{array}\right],
$$

one obtains

$$
Y_{C}-3 a_{22} l=0, Z_{C}+3 a_{32} l=0,
$$

that is

$$
Y_{C}=3 a_{22} l=3 l \cos \psi \cos \varphi=\frac{3}{2} l, Z_{C}=-3 a_{32} l=-3 l \sin \psi \cos \varphi=\frac{3}{2} l .
$$

It results in

$$
\begin{gathered}
{\left[\begin{array}{l}
X_{A_{1}} \\
Y_{A_{1}} \\
Z_{A_{1}}
\end{array}\right]=\left[\begin{array}{c}
0 \\
\frac{3 l}{2} \\
\frac{3 l}{2}
\end{array}\right]+\left[\begin{array}{ccc}
\frac{\sqrt{2}}{2} & \frac{\sqrt{2}}{2} & 0 \\
-\frac{1}{2} & \frac{1}{2} & \frac{\sqrt{2}}{2} \\
\frac{1}{2} & -\frac{1}{2} & \frac{\sqrt{2}}{2}
\end{array}\right]\left[\begin{array}{c}
0 \\
-3 l \\
0
\end{array}\right]=\left[\begin{array}{c}
-\frac{3 l \sqrt{2}}{2} \\
0 \\
3 l
\end{array}\right],} \\
{\left[\begin{array}{c}
X_{A_{2}} \\
Y_{A_{2}} \\
Z_{A_{2}}
\end{array}\right]=\left[\begin{array}{c}
0 \\
\frac{3 l}{2} \\
\frac{3 l}{2}
\end{array}\right]+\left[\begin{array}{ccc}
\frac{\sqrt{2}}{2} & \frac{\sqrt{2}}{2} & 0 \\
-\frac{1}{2} & \frac{1}{2} & \frac{\sqrt{2}}{2} \\
\frac{1}{2} & -\frac{1}{2} & \frac{\sqrt{2}}{2}
\end{array}\right]\left[\begin{array}{c}
0 \\
3 l \\
0
\end{array}\right]=\left[\begin{array}{c}
\frac{3 l \sqrt{2}}{2} \\
0 \\
3 l
\end{array}\right],} \\
{\left[\mathbf{S}_{1}\right]=\left[\begin{array}{ccc}
0 & -3 l & 0 \\
3 l & 0 & \frac{3 l \sqrt{2}}{2} \\
0 & -\frac{3 l \sqrt{2}}{2} & 0
\end{array}\right],\left[\mathbf{S}_{2}\right]=\left[\begin{array}{ccc}
0 & 0 & 3 l \\
0 & 0 & -\frac{3 l \sqrt{2}}{2} \\
-3 l & \frac{3 l \sqrt{2}}{2} & 0
\end{array}\right]} \\
{\left[\mathbf{D}_{1}\right]=\left[\mathbf{S}_{1}\right]\left[\mathbf{R}_{1}\right]=\left[\begin{array}{ccc}
-1.5 & 2.121320 & 1.5 \\
0 & 0 & 3 \\
-1.060660 & 1.5 & 1.060660
\end{array}\right],}
\end{gathered}
$$

$$
\begin{aligned}
& {\left[\mathbf{D}_{2}\right]=\left[\mathbf{S}_{2}\right]\left[\mathbf{R}_{2}\right]=\left[\begin{array}{ccc}
-\frac{3 l \sqrt{2}}{2} & \frac{3 l \sqrt{2}}{2} & 0 \\
\frac{3 l}{2} & -\frac{3 l}{2} & 0 \\
\frac{3 l}{4}(2+\sqrt{2}) & \frac{3 l}{4}(2+\sqrt{2}) & -\frac{3 l}{4}(2-\sqrt{2})
\end{array}\right]=\left[\begin{array}{ccc}
-2.121320 & 2.121320 & 0 \\
1.5 & -1.5 & 0 \\
2.560660 & 2.560660 & -0.621320
\end{array}\right],} \\
& \left\{\mathbf{E}_{1 x}\right\}=\left[\begin{array}{llllll}
-0.5 & 0.5 & 0.707107 & -1.5 & 0 & 4-1.060660
\end{array}\right]^{\mathrm{T}}, \\
& \left\{\mathbf{E}_{2 x}\right\}=\left[\begin{array}{llllll}
-0.5 & 0.5 & 0.707107 & -2.121320 & 1.5 & 2.560660
\end{array}\right]^{\mathrm{T}}, \\
& \left\{\mathbf{E}_{1 y}\right\}=\left[\begin{array}{llllll}
-0.707107 & -0.707107 & 0 & 2.121320 & -2.121320 & 1.5
\end{array}\right]^{\mathrm{T}} \text {, } \\
& \left\{E_{2 y}\right\}=\left[\begin{array}{llllll}
-0.5 & 0.5 & 0.707107 & 2.121320 & -1.5 & 2.560660
\end{array}\right]^{\mathrm{T}},
\end{aligned}
$$

$\left\{\mathbf{E}_{1 z}\right\}=\left[\begin{array}{llllll}0.5 & -0.5 & 0.707107 & 1.5 & 3 & 1.060660\end{array}\right]^{\mathrm{T}},\left\{\mathbf{E}_{2 z}\right\}=\left[\begin{array}{llllll}0.707107 & 0.707107 & 0 & 0 & 0 & -0.621320\end{array}\right]^{\mathrm{T}}$,

$g_{11 x x}=5.5, g_{12 x x}=g_{21 x x}=0, g_{22 x x}=38.242641, g_{11 y x}=-6.363961, g_{12 y x}=32.698485$,

$g_{11 z x}=0, g_{12 z x}=0, g_{21 y x}=-6.863961, g_{22 y x}=-1.757359, g_{21 z x}=0.878680, g_{22 z x}=-2.121320$,

$v_{1 x}^{0}=2.414214, v_{1 y}^{0}=-12.727922, v_{1 z}=6.414214, v_{2 x}^{0}=1.964466, v_{2 y}^{0}=3.035534, v_{2 z}^{0}=10.606602 ;$

- $\quad$ for $k_{1}^{N}=k_{1}^{P}=0, k_{2}^{N}=k_{2}^{P}=0$ (both models, Newton and Poisson):

$$
P_{1}^{N}=0.433941, P_{2}^{N}=0.044318,
$$




$$
\begin{aligned}
& \left\{\mathbf{v}_{C}\right\}=\left[\begin{array}{llllll}
1.993234 & 1.335225 & 0.462374 & 5.239130 & 9.760870 & 1.724495
\end{array}\right]^{\mathrm{T}} \text {, } \\
& v_{1 y}=-8.517194, v_{1 z}=-12.409591, v_{2 y}=6.887904, v_{2 z}=10.319320, \\
& \cos \alpha_{1}^{0}=-0.893012, \sin \alpha_{1}^{0}=0.450032, \alpha_{1}^{0}=153.254263^{\circ}, \\
& \cos \alpha_{2}^{0}=0.275146, \sin \alpha_{2}^{0}=0.961402, \alpha_{2}^{0}=74.029285^{\circ} \text {, } \\
& \cos \alpha_{1}=-0.565879, \sin \alpha_{1}=-0.824488, \alpha_{1}=235.536637^{\circ} \text {, } \\
& \cos \alpha_{2}=0.555267, \sin \alpha_{2}=0.831672, \alpha_{2}=56.270878^{\circ} \text {, } \\
& E_{p N}=0.567345, \Delta E_{c N}=0.567345 \text {; }
\end{aligned}
$$

- $\quad$ for $k_{1}^{N}=k_{1}^{P}=0.6, k_{2}^{N}=k_{2}^{P}=0.3$ :

- Newton model:

$$
\begin{aligned}
& P_{1}^{N}=0.696050, P_{2}^{N}=0.055470, \\
& \left\{\mathbf{v}_{C}\right\}=\left[\begin{array}{llllll}
2.548995 & 1.167944 & 0.794976 & 5.375760 & 9.624240 & 1.547042
\end{array}\right]^{\mathrm{T}} \text {, } \\
& v_{1 y}=-6.484486, v_{1 z}=-23.779531, v_{2 y}=8.909145, v_{2 z}=10.112667 \text {, } \\
& \cos \alpha_{1}^{0}=-0.893012, \sin \alpha_{1}^{0}=0.450032, \alpha_{1}^{0}=153.254263^{\circ}, \\
& \cos \alpha_{2}^{0}=0.275146, \sin \alpha_{2}^{0}=0.961402, \alpha_{2}^{0}=74.029285^{\circ} \text {, } \\
& \cos \alpha_{1}=-0.263086, \sin \alpha_{1}=-0.964772, \alpha_{1}=234.746741^{\circ} \text {, } \\
& \cos \alpha_{2}=0.661046, \sin \alpha_{2}=0.750345, \alpha_{2}=48.620272^{\circ} \text {, } \\
& E_{p N}=1.415162, \Delta E_{c N}=0.374222 ;
\end{aligned}
$$

- Poisson model:

$$
\begin{aligned}
& P_{1}^{N}=0.694306, P_{2}^{N}=0.057614, \\
& \left\{\mathbf{v}_{C}\right\}=\left[\begin{array}{llllll}
2.551569 & 1.135793 & 0.785191 & 5.37596 & 9.624040 & 1.549790
\end{array}\right]^{\mathrm{T}} \text {, } \\
& v_{1 y}=-6.425500, v_{1 z}=-23.703874, v_{2 y}=8.939522, v_{2 z}=10.118746, \\
& \cos \alpha_{1}^{0}=-0.893012, \sin \alpha_{1}^{0}=0.450032, \alpha_{1}^{0}=153.254263^{\circ}, \\
& \cos \alpha_{2}^{0}=0.275146, \sin \alpha_{2}^{0}=0.961402, \alpha_{2}^{0}=74.029285^{\circ} \text {, } \\
& \cos \alpha_{1}=-0.261632, \sin \alpha_{1}=-0.965168, \alpha_{1}=254.833083^{\circ} \text {, } \\
& \cos \alpha_{2}=0.662089, \sin \alpha_{2}=0.749426, \alpha_{2}=48.540681^{\circ} \text {, } \\
& E_{p P}=1.413992, \Delta E_{c P}=0.373592 \text {; } \\
& \text { - } \quad \text { for } k_{1}^{N}=k_{1}^{P}=1.0, k_{2}^{N}=k_{2}^{P}=1.0 \text { (both model): } \\
& P_{1}^{N}=0.867883, P_{2}^{N}=0.088637, \\
& \left\{\mathbf{v}_{C}\right\}=\left[\begin{array}{llllll}
2.986468 & 0.670450 & 0.924747 & 5.478360 & 9.521740 & 1.448990
\end{array}\right]^{\mathrm{T}} \text {, } \\
& v_{1 y}=-4.306466, v_{1 z}=-31.233396, v_{2 y}=10.743873, v_{2 z}=10.032037 \text {, } \\
& \cos \alpha_{1}^{0}=-0.893012, \sin \alpha_{1}^{0}=0.450032, \alpha_{1}^{0}=153.254263^{\circ} \text {, } \\
& \cos \alpha_{2}^{0}=0.275146, \sin \alpha_{2}^{0}=0.961402, \alpha_{2}^{0}=74.029285^{\circ} \text {, } \\
& \cos \alpha_{1}=-0.136588, \sin \alpha_{1}=-0.990628, \alpha_{1}=262.149544^{\circ} \text {, } \\
& \cos \alpha_{2}=0.730905, \sin \alpha_{2}=0.682479, \alpha_{2}=43.037666^{\circ}, \\
& E_{p N}=2.269378, \Delta E_{c P}=0 \text {. }
\end{aligned}
$$


One may also observe that when the two coefficients of restitution at the two points are different, one does not obtain the same results using Newton or Poisson definitions.

\section{Discussions}

Recalling the relations (16), (18), and (19), one may ask for the determination of the situations in which the impulses given by the two models are equal, that is, $P_{1}^{N}=P_{1}^{P}$ and $P_{2}^{N}=P_{2}^{P}$.

These equalities lead to the system

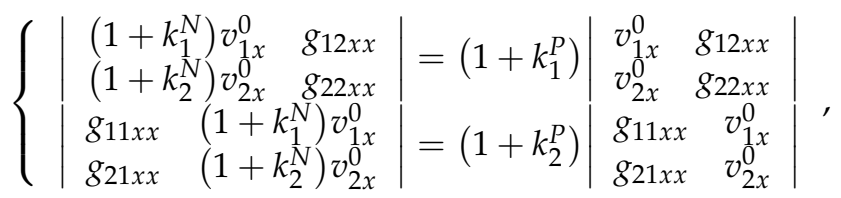

wherefrom

$$
\begin{aligned}
& \left(1+k_{1}^{N}\right) v_{1 x}^{0} g_{22 x x}-\left(1+k_{2}^{N}\right) v_{2 x}^{0} g_{12 x x}=\left(1+k_{1}^{P}\right) v_{1 x}^{0} g_{22 x x}-\left(1+k_{2}^{P}\right) v_{2 x}^{0} g_{12 x x}, \\
& \left(1+k_{2}^{N}\right) v_{2 x}^{0} g_{11 x x}-\left(1+k_{1}^{N}\right) v_{1 x}^{0} g_{21 x x}=\left(1+k_{2}^{P}\right) v_{2 x}^{0} g_{11 x x}-\left(1+k_{2}^{P}\right) v_{1 x}^{0} g_{21 x x} .
\end{aligned}
$$

The previous relations become

$$
\begin{aligned}
& \left(k_{1}^{N}-k_{1}^{P}\right) v_{1 x}^{0} g_{22 x x}-\left(k_{2}^{N}-k_{1}^{P}\right) v_{2 x}^{0} g_{12 x x}=0, \\
& \left(k_{2}^{N}-k_{2}^{P}\right) v_{2 x}^{0} g_{11 x x}-\left(k_{1}^{N}-k_{2}^{P}\right) v_{1 x}^{0} g_{21 x x}=0 .
\end{aligned}
$$

Assuming that $k_{i}^{N}=k_{i}^{P}=k_{i}, i=\overline{1,2}$ (which is valid in the situation of single collision), the Equations (53) and (54) transform into the system

$$
\left\{\begin{array}{l}
\left(k_{2}-k_{1}\right) v_{2 x}^{0} g_{12 x x}=0, \\
\left(k_{2}-k_{1}\right) v_{1 x}^{0} g_{21 x x}=0 .
\end{array}\right.
$$

A first possibility is given by $k_{1}=k_{2}$.

Taking into account that $g_{12 x x}=g_{21 x x}$ and $v_{1 x}^{0} \neq 0, v_{2 x}^{0} \neq 0$ (there exist collisions at the points $A_{1}$ and $A_{2}$ ), the second possibility $g_{12 x x}=g_{21 x x}=0$ results.

From the expression

$$
[\mathbf{\eta}][\mathbf{M}]^{-1}=\left[\begin{array}{cccccc}
m^{-1} & 0 & 0 & 0 & 0 & 0 \\
0 & m^{-1} & 0 & 0 & 0 & 0 \\
0 & 0 & m^{-1} & 0 & 0 & 0 \\
0 & 0 & 0 & J_{x}^{-1} & 0 & 0 \\
0 & 0 & 0 & 0 & J_{y}^{-1} & 0 \\
0 & 0 & 0 & 0 & 0 & J_{z}^{-1}
\end{array}\right]
$$

one gets

$$
g_{i j x x}=\left\{\mathbf{E}_{i x}\right\}^{\mathrm{T}}[\boldsymbol{\eta}][\mathbf{M}]^{-1}\left\{\mathbf{E}_{j x}\right\}=\frac{1}{m}\left(a_{i 1} a_{j 1}+b_{i 1} b_{j 1}+c_{i 1} c_{j 1}\right)+\frac{d_{i 1} d_{j 1}}{J_{x}}+\frac{e_{i 1} e_{j 1}}{J_{y}}+\frac{f_{i 1} f_{j 1}}{J_{z}} ;
$$

since $m>0, J_{x}>0, J_{y}>0, J_{z}>0$, for $i=j$, it results

$$
g_{i i x x}=\frac{1}{m}\left(a_{i 1}^{2}+b_{i 1}^{2}+c_{i 1}^{2}\right)+\frac{d_{i 1}^{2}}{J_{x}}+\frac{e_{i 1}^{2}}{J_{y}}+\frac{f_{i 1}^{2}}{J_{z}}>0
$$

and, consequently, $g_{11 x x}>0, g_{22 x x}>0$. 
If $g_{12 x x}=g_{21 x x}=0, g_{22 x x}>0$, then the relations (16), (18), and (19) lead to

$$
\begin{gathered}
P_{1}^{N}=P_{1}^{P}=P_{1}=\frac{\left|\begin{array}{cc}
\left(1+k_{1}\right) x_{1 x}^{0} & 0 \\
\left(1+k_{2}\right) x_{2 x}^{0} & g_{22 x x}
\end{array}\right|}{\left|\begin{array}{cc}
g_{11 x x} & 0 \\
0 & g_{22 x x}
\end{array}\right|}=\frac{\left(1+k_{1}\right) v_{1 x}^{0}}{g_{11 x x}}, \\
P_{2}^{N}=P_{2}^{P}=P_{2}=\frac{\left|\begin{array}{cc}
g_{11 x x} & \left(1+k_{1}\right) x_{1 x}^{0} \\
0 & \left(1+k_{2}\right) x_{2 x}^{0}
\end{array}\right|}{\left|\begin{array}{cc}
g_{11 x x} & 0 \\
0 & g_{22 x x}
\end{array}\right|}=\frac{\left(1+k_{2}\right) v_{2 x}^{0}}{g_{22 x x}}
\end{gathered}
$$

and since $x_{1 x}^{0}>0, x_{2 x}^{0}>0$, it yields $P_{1}>0, P_{2}>0$.

One may also question the compatibility of the systems (16) and (18).

To answer this question, we have to calculate the determinant

$$
\Delta=\left|\begin{array}{ll}
g_{11 x x} & g_{12 x x} \\
g_{21 x x} & g_{22 x x}
\end{array}\right|=g_{11 x x} g_{22 x x}-g_{21 x x} g_{21 x x}
$$

that is,

$$
\begin{gathered}
\Delta=\left\{\mathbf{E}_{1 x}\right\}^{\mathrm{T}}[\boldsymbol{\eta}][\mathbf{M}]^{-1}\left\{\mathbf{E}_{1 x}\right\}\left\{\mathbf{E}_{2 x}\right\}^{\mathrm{T}}[\boldsymbol{\eta}][\mathbf{M}]^{-1}\left\{\mathbf{E}_{2 x}\right\} \\
-\left\{\mathbf{E}_{1 x}\right\}^{\mathrm{T}}[\boldsymbol{\eta}][\mathbf{M}]^{-1}\left\{\mathbf{E}_{2 x}\right\}\left\{\mathbf{E}_{2 x}\right\}^{\mathrm{T}}[\mathbf{\eta}][\mathbf{M}]^{-1}\left\{\mathbf{E}_{1 x}\right\}= \\
=\left\{\mathbf{E}_{1 x}\right\}^{\mathrm{T}}[\boldsymbol{\eta}][\mathbf{M}]^{-1}\left\{\left\{\mathbf{E}_{1 x}\right\}\left\{\mathbf{E}_{2 x}\right\}^{\mathrm{T}}[\boldsymbol{\eta}][\mathbf{M}]^{-1}\left\{\mathbf{E}_{2 x}\right\}-\left\{\mathbf{E}_{2 x}\right\}\left\{\mathbf{E}_{2 x}\right\}^{\mathrm{T}}[\boldsymbol{\eta}][\mathbf{M}]^{-1}\left\{\mathbf{E}_{1 x}\right\}\right\}= \\
=\left\{\mathbf{E}_{1 x}\right\}^{\mathrm{T}}[\boldsymbol{\eta}][\mathbf{M}]^{-1}\left\{\left\{\mathbf{E}_{1 x}\right\}\left\{\mathbf{E}_{2 x}\right\}^{\mathrm{T}}[\boldsymbol{\eta}][\mathbf{M}]^{-1}\left\{\mathbf{E}_{2 x}\right\}-\left\{\mathbf{E}_{2 x}\right\}\left\{\mathbf{E}_{1 x}\right\}^{\mathrm{T}}[\boldsymbol{\eta}][\mathbf{M}]^{-1}\left\{\mathbf{E}_{2 x}\right\}\right\}= \\
=\left\{\mathbf{E}_{1 x}\right\}^{\mathrm{T}}[\boldsymbol{\eta}][\mathbf{M}]^{-1}\left\{\left\{\mathbf{E}_{1 x}\right\}\left\{\mathbf{E}_{2 x}\right\}^{\mathrm{T}}-\left\{\mathbf{E}_{2 x}\right\}\left\{\mathbf{E}_{1 x}\right\}^{\mathrm{T}}\right\}[\mathbf{\eta}][\mathbf{M}]^{-1}\left\{\mathbf{E}_{2 x}\right\} .
\end{gathered}
$$

One may observe that if $\left\{\mathbf{E}_{1 x}\right\}\left\{\mathbf{E}_{2 x}\right\}^{\mathrm{T}}=\left\{\mathbf{E}_{2 x}\right\}\left\{\mathbf{E}_{1 x}\right\}^{\mathrm{T}}$, which is equivalent to

$$
\begin{aligned}
& {\left[\begin{array}{llllll}
a_{11} a_{21} & a_{11} b_{21} & a_{11} c_{21} & a_{11} d_{21} & a_{11} e_{21} & a_{11} f_{21} \\
b_{11} a_{21} & b_{11} b_{21} & b_{11} c_{21} & b_{11} d_{21} & b_{11} e_{21} & b_{11} f_{21} \\
c_{11} a_{21} & c_{11} b_{21} & c_{11} c_{21} & c_{11} d_{21} & c_{11} e_{21} & c_{11} f_{21} \\
d_{11} a_{21} & d_{11} b_{21} & d_{11} c_{21} & d_{11} d_{21} & d_{11} e_{21} & d_{11} f_{21} \\
e_{11} a_{21} & e_{11} b_{21} & e_{11} c_{21} & e_{11} d_{21} & e_{11} e_{21} & e_{11} f_{21} \\
f_{11} a_{21} & f_{11} b_{21} & f_{11} c_{21} & f_{11} d_{21} & f_{11} e_{21} & f_{11} f_{21}
\end{array}\right]=} \\
& =\left[\begin{array}{llllll}
a_{21} a_{11} & a_{21} b_{11} & a_{21} c_{11} & a_{21} d_{11} & a_{21} e_{11} & a_{21} f_{11} \\
b_{21} a_{11} & b_{21} b_{11} & b_{21} c_{11} & b_{21} d_{11} & b_{21} e_{11} & b_{21} f_{11} \\
c_{21} a_{11} & c_{21} b_{11} & c_{21} c_{11} & c_{21} d_{11} & c_{21} e_{11} & c_{21} f_{11} \\
d_{21} a_{11} & d_{21} b_{11} & d_{21} c_{11} & d_{21} d_{11} & d_{21} e_{11} & d_{21} f_{11} \\
e_{21} a_{11} & e_{21} b_{11} & e_{21} c_{11} & e_{21} d_{11} & e_{21} e_{11} & e_{21} f_{11} \\
f_{21} a_{11} & f_{21} b_{11} & f_{21} c_{11} & f_{21} d_{11} & f_{21} e_{11} & f_{21} f_{11}
\end{array}\right],
\end{aligned}
$$

then $\Delta=0$.

On the other hand, one has

$$
\begin{gathered}
\Delta_{1}^{N}=\left|\begin{array}{cc}
\left(1+k_{1}\right) v_{1 x}^{0} & g_{12 x x} \\
\left(1+k_{2}\right) v_{2 x}^{0} & g_{22 x x}
\end{array}\right|=\left\{\left(1+k_{1}\right) v_{1 x}^{0}\left\{\mathbf{E}_{1 x}\right\}^{\mathrm{T}}-\left(1+k_{2}\right) v_{2 x}^{0}\left\{\mathbf{E}_{2 x}\right\}^{\mathrm{T}}\right\}[\boldsymbol{\eta}][\mathbf{M}]^{-1}\left\{\mathbf{E}_{2 x}\right\}, \\
\Delta_{2}^{N}=\left|\begin{array}{ll}
g_{11 x x} & \left(1+k_{1}\right) v_{1 x}^{0} \\
g_{21 x x} & \left(1+k_{2}\right) v_{2 x}^{0}
\end{array}\right|=\left\{\left(1+k_{2}\right) v_{2 x}^{0}\left\{\mathbf{E}_{1 x}\right\}^{\mathrm{T}}-\left(1+k_{1}\right) v_{1 x}^{0}\left\{\mathbf{E}_{2 x}\right\}^{\mathrm{T}}\right\}[\boldsymbol{\eta}][\mathbf{M}]^{-1}\left\{\mathbf{E}_{1 x}\right\}, \\
\Delta_{1}^{P}=\left|\begin{array}{ll}
v_{1 x}^{0} & g_{12 x x} \\
v_{2 x}^{0} & g_{22 x x}
\end{array}\right|=\left\{v_{1 x}^{0}\left\{\mathbf{E}_{2 x}\right\}^{\mathrm{T}}-v_{2 x}^{0}\left\{\mathbf{E}_{1 x}\right\}^{\mathrm{T}}\right\}[\boldsymbol{\eta}][\mathbf{M}]^{-1}\left\{\mathbf{E}_{2 x}\right\}, \\
\Delta_{2}^{P}=\left|\begin{array}{ll}
g_{11 x x} & v_{1 x}^{0} \\
g_{21 x x} & v_{2 x}^{0}
\end{array}\right|=\left\{v_{2 x}^{0}\left\{\mathbf{E}_{1 x}\right\}^{\mathrm{T}}-v_{1 x}^{0}\left\{\mathbf{E}_{2 x}\right\}^{\mathrm{T}}\right\}[\mathbf{\eta}][\mathbf{M}]^{-1}\left\{\mathbf{E}_{1 x}\right\},
\end{gathered}
$$


Resulting in

$$
P_{1}^{N}=\frac{\Delta_{1}^{N}}{\Delta}, P_{2}^{N}=\frac{\Delta_{2}^{N}}{\Delta}, P_{1}^{P}=\frac{\Delta_{1}^{P}}{\Delta}, P_{2}^{P}=\frac{\Delta_{2}^{P}}{\Delta} .
$$

Let us observe that if the two points $A_{1}$ and $A_{2}$ coincide $\left(A_{1} \equiv A_{2} \equiv A\right)$, then $\left\{\mathbf{E}_{1 x}\right\}=\left\{\mathbf{E}_{2 x}\right\}, v_{1 x}^{0}=v_{2 x}^{0}$, and $k_{1}=k_{2}$, and it results in $\Delta_{1}^{N}=0, \Delta_{1}^{P}=0, \Delta_{2}^{N}=0, \Delta_{2}^{P}=0$, and $\Delta=0$; consequently, the formulae (68) cannot be applied. For the Newton model, we may use the first relation (15), which reads now

$$
\left(1+k_{1}\right) v_{1 x}^{0}=P_{1} g_{11 x x}
$$

that is,

$$
P_{1}=\frac{\left(1+k_{1}\right) v_{1 x}^{0}}{g_{11 x x}} .
$$

For the Poisson model, one has to use the first Equation (17), written as

$$
v_{1 x}^{0}=P_{1}^{c} g_{11 x x},
$$

wherefrom

$$
P_{1}^{c}=\frac{v_{1 x}^{0}}{g_{11 x x}}, P_{1}=\left(1+k_{1}\right) P_{1}^{c}=\frac{\left(1+k_{1}\right) v_{1 x}^{0}}{g_{11 x x}} .
$$

The formulae (70) and (72) are identical to those obtained for the collision of a rigid solid with an impulse.

The conditions $P_{1}^{N}>0, P_{1}^{P}>0, P_{2}^{N}>0, P_{2}^{P}>0$ imply the relations

$$
\Delta_{1}^{N} \Delta>0, \Delta_{2}^{N} \Delta>0, \Delta_{1}^{P} \Delta>0, \Delta_{2}^{N} \Delta>0,
$$

which have the signification of real collisions at the points $A_{1}$ and $A_{2}$.

When $g_{12 x x}=g_{21 x x}=0$, taking into account the expression (59) and (60), the conditions (73) are equivalent to $v_{1 x}^{0}>0$, and $v_{2 x}^{0}>0$.

Remembering the relations (8), we obtain the conditions

$$
\left\{\mathbf{E}_{1 x}\right\}^{\mathrm{T}}[\boldsymbol{\eta}]\left\{\mathbf{v}_{c}^{0}\right\}>0,\left\{\mathbf{E}_{2 x}\right\}^{\mathrm{T}}[\boldsymbol{\eta}]\left\{\mathbf{v}_{c}^{0}\right\}>0,
$$

which are the required conditions in this case.

The expressions (70) show that the model may be applied only if the initial data $\left(\left\{v_{C}^{0}\right\}\right)$ and the matrices $\left\{\mathbf{E}_{1 x}\right\}^{\mathrm{T}}$ and $\left\{\mathbf{E}_{2 x}\right\}^{\mathrm{T}}$ (that is, the geometric positions of the two contact points) satisfy certain inequalities.

In the general case, one has to apply conditions (60), at which one has to add the expressions (62)-(67). These relations define the conditions which must be fulfilled by the initial data. If at least one of these the conditions is not satisfied, then it results that the problem is indeterminate.

More particular cases may be discussed, and some new particular conditions may be obtained.

\section{Collision at Two Points of the Constrained Rigid Solid \\ Determination of the Impulses at the Collision Points}

If the constraints' space is described by the screw matrices of the directions of constraints $\left\{\mathbf{U}_{i}\right\}, i=\overline{1, n}, n$ being the number of constraints, and if the impulses have the scalar values $q_{1}, q_{2}, \ldots, q_{n}$, then $[13,14,43,48,49]$ one may construct the matrix

$$
[\mathbf{U}]=\left[\begin{array}{llll}
\left\{\mathbf{U}_{1}\right\} & \left\{\mathbf{U}_{2}\right\} & \ldots & \left\{\mathbf{U}_{n}\right\}
\end{array}\right],
$$


while the general theorems at collision take the from

$$
[\mathbf{M}]\left\{\left\{\mathbf{V}_{C}\right\}-\left\{\mathbf{V}_{C}^{0}\right\}\right\}=-\sum_{i=1}^{2} P_{i}\left\{\mathbf{E}_{i x}\right\}+[\mathbf{U}]\{\mathbf{q}\},
$$

in which

$$
\{\mathbf{q}\}=\left[\begin{array}{llll}
q_{1} & q_{2} & \ldots & q_{n}
\end{array}\right]^{\mathrm{T}} .
$$

The space of possible velocities is defined by the directions having the plückerian coordinates $\left\{\mathbf{D}_{i}\right\}, i=\overline{1,6-n}$, and by the matrix

$$
[\mathbf{D}]=\left[\begin{array}{llll}
\left\{\mathbf{D}_{1}\right\} & \left\{\mathbf{D}_{2}\right\} & \ldots & \left\{\mathbf{D}_{6-n}\right\}
\end{array}\right],
$$

the velocities along these directions having the scalar values $\xi_{1}, \xi_{2}, \ldots$, and $\xi_{6-n}$.

One obtains the relations

$$
\left\{\mathbf{V}_{C}\right\}=[\mathbf{D}]\{\xi\},\left\{\mathbf{v}_{C}^{0}\right\}=[\mathbf{D}]\left\{\xi^{0}\right\},
$$

in which

$$
\{\xi\}=\left[\begin{array}{llll}
\xi_{1} & \xi_{2} & \ldots & \xi_{6-n}
\end{array}\right]^{\mathrm{T}} .
$$

Relation (76) becomes now

$$
[\mathbf{M}][\mathbf{D}]\left\{\{\xi\}-\left\{\xi^{0}\right\}\right\}=-\sum_{i=1}^{2} P_{i}\left\{\mathbf{E}_{i x}\right\}+[\mathbf{U}]\{\mathbf{q}\} .
$$

Multiplying the last relation by $[\mathbf{D}]^{\mathrm{T}}[\mathbf{\eta}]$, taking into account $[13,14,48,49]$ that

$$
[\mathbf{D}]^{\mathrm{T}}[\mathbf{\eta}][\mathbf{U}]=0
$$

and denoting

$$
\left[\mathbf{M}_{\mathrm{red}}\right]=[\mathbf{D}]^{\mathrm{T}}[\boldsymbol{\eta}][\mathbf{M}][\mathbf{D}]
$$

one obtains

$$
\{\xi\}-\left\{\xi^{0}\right\}=-\sum_{i=1}^{2} P_{i}\left[\mathbf{M}_{\text {red }}\right]^{-1}[\mathbf{D}]^{\mathrm{T}}[\mathbf{\eta}]\left\{\mathbf{E}_{i x}\right\}
$$

or, equivalently,

$$
\left\{\mathbf{V}_{C}\right\}-\left\{\mathbf{V}_{C}^{0}\right\}=-\sum_{i=1}^{2} P_{i}[\mathbf{D}]\left[\mathbf{M}_{\mathrm{red}}\right]^{-1}[\mathbf{D}]^{\mathrm{T}}[\boldsymbol{\eta}]\left\{\mathbf{E}_{i x}\right\}
$$

Considering now the inertances

$$
\begin{gathered}
g_{11 x x}=\left\{\mathbf{E}_{1 x}\right\}^{\mathrm{T}}[\mathbf{\eta}][\mathbf{D}]\left[\mathbf{M}_{\text {red }}\right]^{-1}[\mathbf{D}]^{\mathrm{T}}[\boldsymbol{\eta}]\left\{\mathbf{E}_{1 x}\right\}, \\
g_{12 x x}=\left\{\mathbf{E}_{1 x}\right\}^{\mathrm{T}}[\mathbf{\eta}][\mathbf{D}]\left[\mathbf{M}_{\text {red }}\right]^{-1}[\mathbf{D}]^{\mathrm{T}}[\mathbf{\eta}]\left\{\mathbf{E}_{2 x}\right\}=g_{21 x x}, \\
g_{22 x x}=\left\{\mathbf{E}_{2 x}\right\}^{\mathrm{T}}[\boldsymbol{\eta}][\mathbf{D}]\left[\mathbf{M}_{\text {red }}\right]^{-1}[\mathbf{D}]^{\mathrm{T}}[\boldsymbol{\eta}]\left\{\mathbf{E}_{2 x}\right\},
\end{gathered}
$$

the matrix Equation (85) leads to the system

$$
v_{1 x}-v_{1 x}^{0}=-P_{1} g_{11 x x}-P_{2} g_{12 x x}, v_{2 x}-v_{2 x}^{0}=-P_{1} g_{12 x x}-P_{2} g_{22 x x} .
$$

For the Newton model, one has

$$
\left(1+k_{1}^{N}\right) v_{1 x}^{0}=P_{1} g_{11 x x}+P_{2} g_{12 x x},\left(1+k_{2}^{N}\right) v_{2 x}^{0}=P_{1} g_{21 x x}+P_{2} g_{22 x x},
$$


wherefrom it results in the impulses $P_{1}$ and $P_{2}$

$$
P_{1}=\frac{\left|\begin{array}{cc}
\left(1+k_{1}^{N}\right) v_{1 x}^{0} & g_{12 x x} \\
\left(1+k_{2}^{N}\right) v_{2 x}^{0} & g_{22 x x}
\end{array}\right|}{\left|\begin{array}{ll}
g_{11 x x} & g_{12 x x} \\
g_{21 x x} & g_{22 x x}
\end{array}\right|}, P_{2}=\frac{\left|\begin{array}{ll}
g_{11 x x} & \left(1+k_{1}^{N}\right) v_{1 x}^{0} \\
g_{12 x x} & \left(1+k_{2}^{N}\right) v_{2 x}^{0}
\end{array}\right|}{\left|\begin{array}{ll}
g_{11 x x} & g_{12 x x} \\
g_{21 x x} & g_{22 x x}
\end{array}\right|} .
$$

From the expression (81), one obtains

$$
[\mathbf{D}]\left\{\{\xi\}-\left\{\xi^{0}\right\}\right\}=-\sum_{i=1}^{2} P_{i}[\mathbf{M}]^{-1}\left\{\mathbf{E}_{i x}\right\}+[\mathbf{M}]^{-1}[\mathbf{U}]\{\mathbf{q}\}
$$

and, by multiplication by $[\mathbf{U}]^{\mathrm{T}}[\boldsymbol{\eta}]$ and taking into account that $[13,14,48,49]$

$$
[\mathbf{U}]^{\mathrm{T}}[\boldsymbol{\eta}][\mathbf{D}]=0,
$$

it yields

$$
[\mathbf{U}]^{\mathrm{T}}[\mathbf{\eta}][\mathbf{M}]^{-1}[\mathbf{U}]\{\mathbf{q}\}=\sum_{i=1}^{2} P_{i}[\mathbf{U}]^{\mathrm{T}}[\mathbf{\eta}][\mathbf{M}]^{-1}\left\{\mathbf{E}_{i x}\right\}
$$

wherefrom

$$
\{\mathbf{q}\}=\sum_{i=1}^{2} P_{i}\left[[\mathbf{U}]^{\mathrm{T}}[\mathbf{\eta}][\mathbf{M}]^{-1}[\mathbf{U}]\right]^{-1}[\mathbf{U}]^{\mathrm{T}}[\boldsymbol{\eta}][\mathbf{M}]^{-1}\left\{\mathbf{E}_{i x}\right\}[\mathbf{U}]^{\mathrm{T}}[\mathbf{\eta}][\mathbf{M}]^{-1}\left\{\mathbf{E}_{i x}\right\}
$$

\section{Velocities after Collision}

Knowing the impulses $P_{1}$ and $P_{2}$ (Equation (89)), from the matrix relation (85) one deduces $\left\{\mathbf{V}_{C}\right\}$, while the system (87) yields $v_{1 x}$ and $v_{2 x}$.

Further on, using the inertances

$$
\begin{gathered}
g_{11 y x}=\left\{\mathbf{E}_{1 y}\right\}^{\mathrm{T}}[\boldsymbol{\eta}][\mathbf{D}]\left[\mathbf{M}_{\text {red }}\right]^{-1}[\mathbf{D}]^{\mathrm{T}}[\mathbf{\eta}]\left\{\mathbf{E}_{1 x}\right\}, \\
g_{12 y x}=g_{21 y x}=\left\{\mathbf{E}_{1 y}\right\}^{\mathrm{T}}[\mathbf{\eta}][\mathbf{D}]\left[\mathbf{M}_{\mathrm{red}}\right]^{-1}[\mathbf{D}]^{\mathrm{T}}[\mathbf{\eta}]\left\{\mathbf{E}_{2 x}\right\}, \\
g_{22 y x}=\left\{\mathbf{E}_{2 y}\right\}^{\mathrm{T}}[\boldsymbol{\eta}][\mathbf{D}]\left[\mathbf{M}_{\mathrm{red}}\right]^{-1}[\mathbf{D}]^{\mathrm{T}}[\mathbf{\eta}]\left\{\mathbf{E}_{2 x}\right\}, \\
g_{11 z x}=\left\{\mathbf{E}_{1 z}\right\}^{\mathrm{T}}[\boldsymbol{\eta}][\mathbf{D}]\left[\mathbf{M}_{\mathrm{red}}\right]^{-1}[\mathbf{D}]^{\mathrm{T}}[\boldsymbol{\eta}]\left\{\mathbf{E}_{1 x}\right\}, \\
g_{11 z x}=g_{21 z x}=\left\{\mathbf{E}_{1 z}\right\}^{\mathrm{T}}[\boldsymbol{\eta}][\mathbf{D}]\left[\mathbf{M}_{\mathrm{red}}\right]^{-1}[\mathbf{D}]^{\mathrm{T}}[\boldsymbol{\eta}]\left\{\mathbf{E}_{2 x}\right\}, \\
g_{22 z x}=\left\{\mathbf{E}_{2 z}\right\}^{\mathrm{T}}[\mathbf{\eta}][\mathbf{D}]\left[\mathbf{M}_{\text {red }}\right]^{-1}[\mathbf{D}]^{\mathrm{T}}[\mathbf{\eta}]\left\{\mathbf{E}_{2 x}\right\},
\end{gathered}
$$

from the matrix relation (85), one obtains the velocities

$$
\begin{aligned}
& v_{1 y}=v_{1 y}^{0}-P_{1} g_{11 y x}-P_{2} g_{12 y x}, v_{2 y}=v_{2 y}^{0}-P_{1} g_{21 y x}-P_{2} g_{22 y x}, \\
& v_{1 z}=v_{1 z}^{0}-P_{1} g_{11 z x}-P_{2} g_{22 z x}, v_{2 z}=v_{2 z}^{0}-P_{1} g_{21 z x}-P_{2} g_{22 z x} .
\end{aligned}
$$

The energy of the lost velocities reads

$$
E_{p}=\frac{1}{2}\left(P_{1}^{2} g_{11 x x}+2 P_{1} P_{2} g_{12 x x}+P_{2}^{2} g_{22 x x}\right)
$$

while the variation of the kinetic energy is

$$
\Delta E_{c}=-E_{p}+P_{1} v_{1 x}^{0}+P_{2} v_{2 x}^{0} .
$$




\section{Examples}

Example 5. The homogeneous bar OA (Figure 7) collides with two obstacles at the points $A_{1}$ and $A_{2}$.

One knows: the mass $m$ of the bar, the dimensions $a, b, l$, the coefficients of restitution $k_{1}$ and $k_{2}$ at the points $A_{1}$ and $A_{2}$, respectively, and the initial distribution of velocities, $v_{X}=0, v_{Y}=0, v_{Z}=0, \omega_{X}=0, \omega_{Y}=0, \omega_{Z}=\omega$.

The only possible motion is the rotation about the $O Z$ axis, and because $\mathbf{C O} \times \mathbf{k}=-l \mathbf{i}$, one obtains

$$
\{\mathbf{D}\}=[\mathbf{D}]=\left[\begin{array}{llllll}
0 & 0 & 1 & -l & 0 & 0
\end{array}\right]^{\mathrm{T}} \text {. }
$$

The constraints are:

- $\quad$ displacement along the $O X$ axis; since $\mathbf{C O} \times \mathbf{i}=l \mathbf{k}$, the following results

$$
\left\{\mathbf{U}_{1}\right\}=\left[\begin{array}{llllll}
1 & 0 & 0 & 0 & 0 & l
\end{array}\right]^{\mathrm{T}}
$$

- $\quad$ displacement along the OY axis; we have $\mathbf{C O} \times \mathbf{j}=0$, hence

$$
\left\{\mathbf{U}_{2}\right\}=\left[\begin{array}{llllll}
0 & 1 & 0 & 0 & 0 & 0
\end{array}\right]^{\mathrm{T}} \text {; }
$$

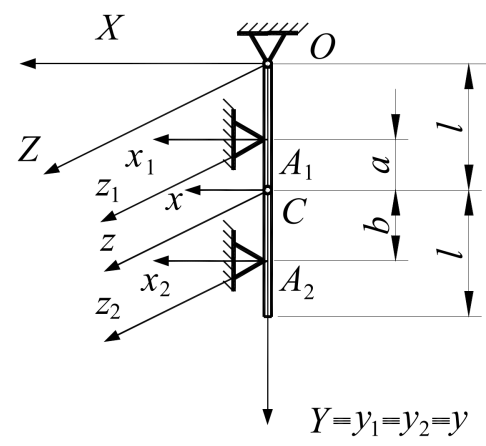

Figure 7. Example 5.

- displacement along the $O Z$ axis; $\mathbf{C O} \times \mathbf{k}=-\mathbf{l} \mathbf{i}$, therefore

$$
\left\{\mathbf{U}_{3}\right\}=\left[\begin{array}{llllll}
0 & 0 & 1 & -l & 0 & 0
\end{array}\right]^{\mathrm{T}} ;
$$

- $\quad$ rotation about the $O X$ axis;

$$
\left\{\mathbf{U}_{4}\right\}=\left[\begin{array}{llllll}
0 & 0 & 0 & 1 & 0 & 0
\end{array}\right]^{\mathrm{T}} \text {; }
$$

- $\quad$ rotation about the OY axis;

$$
\left\{\mathbf{U}_{5}\right\}=\left[\begin{array}{llllll}
0 & 0 & 0 & 0 & 1 & 0
\end{array}\right]^{\mathrm{T}} \text {; }
$$

in conclusion

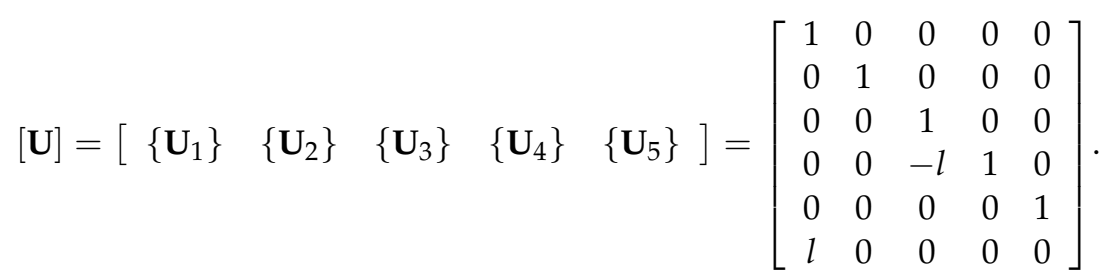




\section{It is obvious that}

$$
[\mathbf{D}]^{\mathrm{T}}[\boldsymbol{\eta}][\mathbf{U}]=\left[\begin{array}{lllll}
0 & 0 & 0 & 0 & 0
\end{array}\right] .
$$

We have

$$
\begin{aligned}
& {\left[\mathbf{S}_{1}\right]=\left[\begin{array}{ccc}
0 & 0 & -a \\
0 & 0 & 0 \\
a & 0 & 0
\end{array}\right],\left[\mathbf{S}_{2}\right]=\left[\begin{array}{ccc}
0 & 0 & b \\
0 & 0 & 0 \\
-b & 0 & 0
\end{array}\right],\left[\mathbf{R}_{1}\right]=\left[\begin{array}{lll}
1 & 0 & 0 \\
0 & 1 & 0 \\
0 & 0 & 1
\end{array}\right],\left[\mathbf{R}_{2}\right]=\left[\begin{array}{lll}
1 & 0 & 0 \\
0 & 1 & 0 \\
0 & 0 & 1
\end{array}\right],} \\
& {\left[\mathbf{D}_{1}\right]=\left[\mathbf{S}_{1}\right]\left[\mathbf{R}_{1}\right]=\left[\begin{array}{ccc}
0 & 0 & -a \\
0 & 0 & 0 \\
a & 0 & 0
\end{array}\right],\left[\mathbf{D}_{2}\right]=\left[\mathbf{S}_{2}\right]\left[\mathbf{R}_{2}\right]=\left[\begin{array}{ccc}
0 & 0 & b \\
0 & 0 & 0 \\
-b & 0 & 0
\end{array}\right],} \\
& \left\{\mathbf{E}_{1 x}\right\}=\left[\begin{array}{llllll}
1 & 0 & 0 & 0 & 0 & a
\end{array}\right]^{\mathrm{T}},\left\{\mathbf{E}_{1 y}\right\}=\left[\begin{array}{llllll}
0 & 1 & 0 & 0 & 0 & 0
\end{array}\right]^{\mathrm{T}},\left\{\mathbf{E}_{1 z}\right\}=\left[\begin{array}{llllll}
0 & 0 & 1 & -a & 0 & 0
\end{array}\right]^{\mathrm{T}}, \\
& \left\{\mathbf{E}_{2 x}\right\}=\left[\begin{array}{llllll}
1 & 0 & 0 & 0 & 0 & -b
\end{array}\right]^{\mathrm{T}},\left\{\mathbf{E}_{2 y}\right\}=\left[\begin{array}{llllll}
0 & 1 & 0 & 0 & 0 & 0
\end{array}\right]^{\mathrm{T}},\left\{\mathbf{E}_{2 z}\right\}=\left[\begin{array}{llllll}
0 & 0 & 1 & b & 0 & 0
\end{array}\right]^{\mathrm{T}}, \\
& {[\boldsymbol{\eta}]=\left[\begin{array}{llllll}
0 & 0 & 0 & 1 & 0 & 0 \\
0 & 0 & 0 & 0 & 1 & 0 \\
0 & 0 & 0 & 0 & 0 & 1 \\
1 & 0 & 0 & 0 & 0 & 0 \\
0 & 1 & 0 & 0 & 0 & 0 \\
0 & 0 & 1 & 0 & 0 & 0
\end{array}\right]} \\
& {[\mathbf{M}]=\left[\begin{array}{cccccc}
0 & 0 & 0 & m & 0 & 0 \\
0 & 0 & 0 & 0 & m & 0 \\
0 & 0 & 0 & 0 & 0 & m \\
J_{x} & 0 & 0 & 0 & 0 & 0 \\
0 & J_{y} & 0 & 0 & 0 & 0 \\
0 & 0 & J_{z} & 0 & 0 & 0
\end{array}\right],[\mathbf{M}]^{-1}=\left[\begin{array}{cccccc}
0 & 0 & 0 & J_{x}^{-1} & 0 & 0 \\
0 & 0 & 0 & 0 & J_{y}^{-1} & 0 \\
0 & 0 & 0 & 0 & 0 & J_{z}^{-1} \\
m^{-1} & 0 & 0 & 0 & 0 & 0 \\
0 & m^{-1} & 0 & 0 & 0 & 0 \\
0 & 0 & m^{-1} & 0 & 0 & 0
\end{array}\right],} \\
& {\left[\mathbf{M}_{\mathrm{red}}\right]=[\mathbf{D}]^{\mathrm{T}}[\mathbf{\eta}][\mathbf{M}][\mathbf{D}]=\left[m l^{2}+J_{z}\right]=J_{O},\left[\mathbf{M}_{\mathrm{red}}\right]^{-1}=\left[\frac{1}{J_{O}}\right]=\frac{1}{J_{O}},} \\
& g_{11 x x}=\left\{\mathbf{E}_{1 x}\right\}^{\mathrm{T}}[\boldsymbol{\eta}][\mathbf{D}]\left[\mathbf{M}_{\mathrm{red}}\right]^{-1}[\mathbf{D}]^{\mathrm{T}}[\boldsymbol{\eta}]\left\{\mathbf{E}_{1 x}\right\}=\frac{(l-a)^{2}}{J_{O}}, \\
& g_{12 x x}=\left\{\mathbf{E}_{1 x}\right\}^{\mathrm{T}}[\mathbf{\eta}][\mathbf{D}]\left[\mathbf{M}_{\mathrm{red}}\right]^{-1}[\mathbf{D}]^{\mathrm{T}}[\mathbf{\eta}]\left\{\mathbf{E}_{2 x}\right\}=\frac{(l-a)(l+b)}{J_{O}}=g_{21 x x}, \\
& g_{22 x x}=\left\{\mathbf{E}_{2 x}\right\}^{\mathrm{T}}[\mathbf{\eta}][\mathbf{D}]\left[\mathbf{M}_{\text {red }}\right]^{-1}[\mathbf{D}]^{\mathrm{T}}[\mathbf{\eta}]\left\{\mathbf{E}_{2 x}\right\}=\frac{(l+b)^{2}}{J O}, \\
& \left\{\mathbf{v}_{C}^{0}\right\}=\left[\begin{array}{llllll}
0 & 0 & \omega & 0 & 0 & 0
\end{array}\right]^{\mathrm{T}} \text {, } \\
& \left\{\mathbf{v}_{1 x}^{0}\right\}=\left\{\mathbf{E}_{1 x}\right\}^{\mathrm{T}}[\boldsymbol{\eta}]\left\{\mathbf{v}_{C}^{0}\right\}=\omega a,\left\{\mathbf{v}_{1 y}^{0}\right\}=\left\{\mathbf{E}_{1 y}\right\}^{\mathrm{T}}[\mathbf{\eta}]\left\{\mathbf{v}_{C}^{0}\right\}=0,\left\{\mathbf{v}_{1 z}^{0}\right\}=\left\{\mathbf{E}_{1 z}\right\}^{\mathrm{T}}[\mathbf{\eta}]\left\{\mathbf{v}_{C}^{0}\right\}=0, \\
& \left\{\mathbf{v}_{2 x}^{0}\right\}=\left\{\mathbf{E}_{2 x}\right\}^{\mathrm{T}}[\boldsymbol{\eta}]\left\{\mathbf{v}_{C}^{0}\right\}=-b \boldsymbol{w},\left\{\mathbf{v}_{2 y}^{0}\right\}=\left\{\mathbf{E}_{2 y}\right\}^{\mathrm{T}}[\boldsymbol{\eta}]\left\{\mathbf{v}_{C}^{0}\right\}=0,\left\{\mathbf{v}_{2 z}^{0}\right\}=\left\{\mathbf{E}_{2 z}\right\}^{\mathrm{T}}[\boldsymbol{\eta}]\left\{\mathbf{v}_{C}^{0}\right\}=0, \\
& \left|\begin{array}{ll}
g_{11 x x} & g_{12 x x} \\
g_{21 x x} & g_{22 x x}
\end{array}\right|=0
\end{aligned}
$$

The system (88) is: 
- $\quad$ incompatible if the rank of the extended matrix

$$
\left[\begin{array}{c}
\mathbf{A}
\end{array}\right]=\left[\begin{array}{lll}
g_{11 x x} & g_{12 x x} & \left(1+k_{1}^{N}\right) v_{1 x}^{0} \\
g_{21 x x} & g_{22 x x} & \left(1+k_{2}^{N}\right) v_{2 x}^{0}
\end{array}\right]
$$

is equal to 2 , that is either

$$
\left|\begin{array}{ll}
g_{11 x x} & \left(1+k_{1}^{N}\right) v_{1 x}^{0} \\
g_{21 x x} & \left(1+k_{2}^{N}\right) v_{2 x}^{0}
\end{array}\right| \neq 0
$$

or

$$
\left|\begin{array}{cc}
\left(1+k_{1}^{N}\right) v_{1 x}^{0} & g_{12 x x} \\
\left(1+k_{2}^{N}\right) v_{2 x}^{0} & g_{22 x x}
\end{array}\right| \neq 0
$$

The relation (98) leads to

$$
\left(1+k_{1}^{N}\right) a \omega \frac{(l+b)^{2}}{J_{O}} \neq\left(1+k_{2}^{N}\right)(-b \omega) \frac{(l-b)(l+b)}{J_{O}},
$$

wherefrom

$$
\left(1+k_{1}^{N}\right) a(l+b) \neq-\left(1+k_{2}^{N}\right) b(l-a) .
$$

Analogically, expression (99) offers

$$
\left(1+k_{1}^{N}\right) a \omega \frac{(l+b)^{2}}{J_{O}} \neq\left(1+k_{2}^{N}\right)(-b \omega) \frac{(l-b)(l+b)}{J_{O}},
$$

wherefrom one gets the same relation (100);

- compatible if rank $[\overline{\mathbf{A}}]=1$, that is

$$
\left(1+k_{1}^{N}\right) a(l+b)=-\left(1+k_{2}^{N}\right) b(l-a),
$$

wherefrom

$$
k_{2}^{N}=\frac{b l-a b-\left(1+k_{1}^{N}\right)(a b+a l)}{b l+a b} .
$$

For instance, if $a=b=\frac{l}{2}$, then it results in

$$
k_{2}^{N}=\frac{1}{3}-K_{1}^{N}
$$

Since $k_{1}^{N} \geq 0$ and $k_{2}^{N} \geq 0$, one obtained, in this particular case, $k_{1}^{N} \leq \frac{1}{3}, k_{2}^{N} \leq \frac{1}{3}$.

Remark 1. One may observe that $\left\{\mathbf{E}_{1 x}\right\}\left\{\mathbf{E}_{2 x}\right\}^{\mathrm{T}} \neq\left\{\mathbf{E}_{2 x}\right\}\left\{\mathbf{E}_{1 x}\right\}^{\mathrm{T}}$, and

$$
\left|\begin{array}{ll}
g_{11 x x} & g_{12 x x} \\
g_{21 x x} & g_{22 x x}
\end{array}\right|=0
$$

The resulting condition (63) is a sufficient but not necessary one for the incompatibility of the system which gives the impulses.

Example 6. Let us modify the previous example assuming that the end $O$ of the bar can move along the $O X$ axis (Figure 8). 


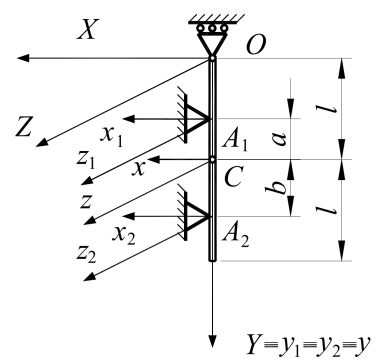

Figure 8. Example 6.

In this situation, the bar may rotate about the axis,

$$
\left\{\mathbf{D}_{1}\right\}=\left[\begin{array}{llllll}
0 & 0 & 1 & -l & 0 & 0
\end{array}\right]^{\mathrm{T}},
$$

and it may translate along the $O X$ axis,

$$
\left\{\mathbf{D}_{2}\right\}=\left[\begin{array}{llllll}
0 & 0 & 0 & 1 & 0 & 0
\end{array}\right]^{\mathrm{T}} ;
$$

resulting in

$$
[\mathbf{D}]=\left[\begin{array}{ll}
\left\{\mathbf{D}_{1}\right\} & \left\{\mathbf{D}_{2}\right\}
\end{array}\right]=\left[\begin{array}{cc}
0 & 0 \\
0 & 0 \\
1 & 0 \\
-l & 1 \\
0 & 0 \\
0 & 0
\end{array}\right]
$$

Analogically, the constraints are:

- the translation along the OY axis,

$$
\left\{\mathbf{U}_{1}\right\}=\left[\begin{array}{llllll}
0 & 1 & 0 & 0 & 0 & 0
\end{array}\right]^{\mathrm{T}} ;
$$

- $\quad$ the translation along the OZ axis,

$$
\left\{\mathbf{U}_{2}\right\}=\left[\begin{array}{llllll}
0 & 0 & 1 & -l & 0 & 0
\end{array}\right]^{\mathrm{T}} ;
$$

- $\quad$ the rotation about the $O X$ axis,

$$
\left\{\mathbf{U}_{3}\right\}=\left[\begin{array}{llllll}
0 & 0 & 0 & 1 & 0 & 0
\end{array}\right]^{\mathrm{T}} ;
$$

- the rotation about the OY axis,

$$
\left\{\mathbf{U}_{4}\right\}=\left[\begin{array}{llllll}
0 & 0 & 0 & 0 & 1 & 0
\end{array}\right]^{\mathrm{T}} ;
$$

one gets

$$
[\mathbf{U}]=\left[\begin{array}{llll}
\left\{\mathbf{U}_{1}\right\} & \left\{\mathbf{U}_{2}\right\} & \left\{\mathbf{U}_{3}\right\} & \left\{\mathbf{U}_{4}\right\}
\end{array}\right]=\left[\begin{array}{cccc}
0 & 0 & 0 & 0 \\
1 & 0 & 0 & 0 \\
0 & 1 & 0 & 0 \\
0 & -l & 1 & 0 \\
0 & 0 & 0 & 1 \\
0 & 0 & 0 & 0
\end{array}\right]
$$

A simple calculation offers

$$
[\mathbf{D}]^{\mathrm{T}}[\boldsymbol{\eta}][\mathbf{U}]=\left[\begin{array}{llll}
0 & 0 & 0 & 0 \\
0 & 0 & 0 & 0
\end{array}\right]=[0] .
$$




\section{One obtains}

$$
\begin{aligned}
& \left\{\mathbf{E}_{1 x}\right\}=\left[\begin{array}{llllll}
1 & 0 & 0 & 0 & 0 & 0
\end{array}\right]^{\mathrm{T}},\left\{\mathbf{E}_{1 y}\right\}=\left[\begin{array}{llllll}
0 & 1 & 0 & 0 & 0 & 0
\end{array}\right]^{\mathrm{T}},\left\{\mathbf{E}_{1 z}\right\}=\left[\begin{array}{llllll}
0 & 0 & 1 & -a & 0 & 0
\end{array}\right]^{\mathrm{T}}, \\
& \left\{\mathbf{E}_{2 x}\right\}=\left[\begin{array}{llllll}
1 & 0 & 0 & 0 & 0 & -b
\end{array}\right]^{\mathrm{T}},\left\{\mathbf{E}_{2 y}\right\}=\left[\begin{array}{llllll}
0 & 1 & 0 & 0 & 0 & 0
\end{array}\right]^{\mathrm{T}},\left\{\mathbf{E}_{2 z}\right\}=\left[\begin{array}{llllll}
0 & 0 & 1 & b & 0 & 0
\end{array}\right]^{\mathrm{T}}, \\
& {\left[\mathbf{M}_{\mathrm{red}}\right]=[\mathbf{D}]^{\mathrm{T}}[\mathbf{\eta}][\mathbf{M}][\mathbf{D}]=\left[\begin{array}{cc}
J_{z}+m l^{2} & -m l \\
-m l & m
\end{array}\right],\left[\mathbf{M}_{\mathrm{red}}\right]^{-1}=\frac{1}{m J_{z}}\left[\begin{array}{cc}
m & m l \\
m l & J_{z}+m l^{2}
\end{array}\right]} \\
& g_{11 x x}=\left\{\mathbf{E}_{1 x}\right\}^{\mathrm{T}}[\boldsymbol{\eta}][\mathbf{D}]\left[\mathbf{M}_{\text {red }}\right]^{-1}[\mathbf{D}]^{\mathrm{T}}[\boldsymbol{\eta}]\left\{\mathbf{E}_{1 x}\right\}=\frac{J_{z}+m a^{2}}{m J_{z}}, \\
& g_{12 x x}=\left\{\mathbf{E}_{1 x}\right\}^{\mathrm{T}}[\boldsymbol{\eta}][\mathbf{D}]\left[\mathbf{M}_{\text {red }}\right]^{-1}[\mathbf{D}]^{\mathrm{T}}[\boldsymbol{\eta}]\left\{\mathbf{E}_{2 x}\right\}=\frac{J_{z}-m a b}{m J_{z}}=g_{21 x x}, \\
& g_{22 x x}=\left\{\mathbf{E}_{2 x}\right\}^{\mathrm{T}}[\boldsymbol{\eta}][\mathbf{D}]\left[\mathbf{M}_{\mathrm{red}}\right]^{-1}[\mathbf{D}]^{\mathrm{T}}[\boldsymbol{\eta}]\left\{\mathbf{E}_{2 x}\right\}=\frac{I_{z}+m b^{2}}{m J_{z}}, \\
& \left\{\mathbf{v}_{C}^{0}\right\}=\left[\begin{array}{llllll}
0 & 0 & \omega & 0 & 0 & 0
\end{array}\right]^{\mathrm{T}} \text {, } \\
& v_{1 x}^{0}=\left\{\mathbf{E}_{1 x}\right\}^{\mathrm{T}}[\boldsymbol{\eta}]\left\{\mathbf{v}_{C}^{0}\right\}=a \boldsymbol{\omega}, v_{1 y}^{0}=\left\{\mathbf{E}_{1 y}\right\}^{\mathrm{T}}[\boldsymbol{\eta}]\left\{\mathbf{v}_{C}^{0}\right\}=0, v_{1 z}^{0}=\left\{\mathbf{E}_{1 x}\right\}^{\mathrm{T}}[\boldsymbol{\eta}]\left\{\mathbf{v}_{C}^{0}\right\}=0, \\
& v_{2 x}^{0}=\left\{\mathbf{E}_{2 x}\right\}^{\mathrm{T}}[\boldsymbol{\eta}]\left\{\mathbf{v}_{C}^{0}\right\}=-b \boldsymbol{\omega}, v_{2 y}^{0}=\left\{\mathbf{E}_{2 y}\right\}^{\mathrm{T}}[\boldsymbol{\eta}]\left\{\mathbf{v}_{C}^{0}\right\}=0, v_{2 z}^{0}=\left\{\mathbf{E}_{2 x}\right\}^{\mathrm{T}}[\boldsymbol{\eta}]\left\{\mathbf{v}_{C}^{0}\right\}=0, \\
& \Delta=\left|\begin{array}{ll}
g_{11 x x} & g_{12 x x} \\
g_{21 x x} & g_{22 x x}
\end{array}\right|=\frac{(a+b)^{2}}{m J_{z}} \\
& \left|\begin{array}{cc}
\left(1+k_{1}^{N}\right) v_{1 x}^{0} & g_{12 x x} \\
\left(1+k_{2}^{N}\right) v_{2 x}^{0} & g_{22 x x}
\end{array}\right|=\frac{\left(J_{z}+m b^{2}\right)\left(1+k_{1}^{N}\right) a \omega+\left(J_{z}-m a b\right)\left(1+k_{2}^{N}\right) b \omega}{m J_{z}}, \\
& \begin{array}{ll}
g_{11 x x} & \left(1+k_{1}^{N}\right) v_{1 x}^{0} \\
g_{21 x x} & \left(1+k_{2}^{N}\right) v_{2 x}^{0}
\end{array} \mid=-\frac{\left(J_{z}+m a^{2}\right)\left(1+k_{2}^{N}\right) b \omega-\left(J_{z}-m a b\right)\left(1+k_{1}^{N}\right) a \omega}{m J_{z}}, \\
& P_{1}=\frac{\left(J_{z}+m b^{2}\right)\left(1+k_{1}^{N}\right) a \omega-\left(J_{z}-m a b\right)\left(1+k_{2}^{N}\right) b \omega}{(a+b)^{2}}, P_{2}=-\frac{-\left(J_{z}+m a^{2}\right)\left(1+k_{2}^{N}\right) b \omega-\left(J_{z}-m a b\right)\left(1+k_{1}^{N}\right) a \omega}{(a+b)^{2}} .
\end{aligned}
$$

Further on, for the simplicity of the calculation, we will consider that $a=b=\frac{l}{3}$. It results in

$$
\begin{gathered}
J_{z}=\frac{m l^{2}}{3}, \\
g_{11 x x}=\frac{4}{3 m}, g_{12 x x}=g_{21 x x}=\frac{2}{3 m}, g_{22 x x}=\frac{4}{3 m} \\
\Delta=\frac{4}{3 m^{2}}, \\
P_{1}=\frac{1}{12} m l \omega\left[4\left(1+k_{1}^{N}\right)+2\left(1+k_{2}^{N}\right)\right], P_{2}=-\frac{1}{12} m l \omega\left[4\left(1+k_{2}^{N}\right)+2\left(1+k_{1}^{N}\right)\right], \\
v_{1 x}=v_{1 x}^{0}-P_{1} g_{11 x x}-P_{2} g_{12 x x}=\frac{l \omega}{18}\left[6-6\left(1+k_{1}\right)\right], \\
v_{2 x}=v_{2 x}^{0}-P_{1} g_{12 x x}-P_{2} g_{22 x x}=\frac{l \omega}{18}\left[-6+6\left(1+k_{2}^{N}\right)\right],
\end{gathered}
$$

$g_{11 y x}=\left\{\mathbf{E}_{1 y}\right\}^{\mathrm{T}}[\boldsymbol{\eta}][\mathbf{D}]\left[\mathbf{M}_{\text {red }}\right]^{-1}[\mathbf{D}]^{\mathrm{T}}[\boldsymbol{\eta}]\left\{\mathbf{E}_{1 x}\right\}=0, g_{12 y x}=g_{21 y x}=\left\{\mathbf{E}_{1 y}\right\}^{\mathrm{T}}[\boldsymbol{\eta}][\mathbf{D}]\left[\mathbf{M}_{\mathrm{red}}\right]^{-1}[\mathbf{D}]^{\mathrm{T}}[\boldsymbol{\eta}]\left\{\mathbf{E}_{2 x}\right\}=0$,

$g_{22 y x}=\left\{\mathbf{E}_{2 y}\right\}^{\mathrm{T}}[\boldsymbol{\eta}][\mathbf{D}]\left[\mathbf{M}_{\text {red }}\right]^{-1}[\mathbf{D}]^{\mathrm{T}}[\boldsymbol{\eta}]\left\{\mathbf{E}_{2 x}\right\}=0, g_{11 z x}=\left\{\mathbf{E}_{1 z}\right\}^{\mathrm{T}}[\boldsymbol{\eta}][\mathbf{D}]\left[\mathbf{M}_{\mathrm{red}}\right]^{-1}[\mathbf{D}]^{\mathrm{T}}[\boldsymbol{\eta}]\left\{\mathbf{E}_{1 x}\right\}=0$, $g_{12 z x}=g_{21 z x}=\left\{\mathbf{E}_{1 z}\right\}^{\mathrm{T}}[\boldsymbol{\eta}][\mathbf{D}]\left[\mathbf{M}_{\mathrm{red}}\right]^{-1}[\mathbf{D}]^{\mathrm{T}}[\boldsymbol{\eta}]\left\{\mathbf{E}_{1 x}\right\}=0, g_{22 z x}=\left\{\mathbf{E}_{2 z}\right\}^{\mathrm{T}}[\boldsymbol{\eta}][\mathbf{D}]\left[\mathbf{M}_{\text {red }}\right]^{-1}[\mathbf{D}]^{\mathrm{T}}[\boldsymbol{\eta}]\left\{\mathbf{E}_{2 x}\right\}=0$,

$$
v_{1 y}=v_{1 y}^{0}-P_{1} g_{11 y x}-P_{2} g_{12 y x}=0, v_{2 y}=v_{2 y}^{0}-P_{1} g_{21 y x}-P_{2 g_{22 y x}}=0,
$$




$$
\begin{aligned}
& v_{1 z}=v_{1 z}^{0}-P_{1} g_{11 z x}-P_{2} g_{12 z x}=0, v_{2 z}=v_{2 z}^{0}-P_{1} g_{21 z x}-P_{2} g_{22 z x}=0, \\
& E_{p}=\frac{1}{2}\left(P_{1}^{2} g_{11 x x}+2 P_{1} P_{2} g_{12 x x}+P_{2}^{2} g_{22 x x}\right) \\
& =\frac{m l^{2} \omega^{2}}{316}\left\{\left[4\left(1+k_{1}^{N}\right)+2\left(1+k_{2}^{N}\right)\right]^{2}+\left[4\left(1+k_{1}^{N}\right)+2\left(1+k_{2}^{N}\right)\right]\left[4\left(1+k_{2}^{N}\right)+2\left(1+k_{1}^{N}\right)\right]\right. \\
& \left.+\left[4\left(1+k_{2}^{N}\right)+2\left(1+k_{1}^{N}\right)\right]^{2}\right\}, \\
& \Delta E_{c}=-E_{p}+P_{1} v_{1 x}^{0}+P_{2} v_{2 x}^{0} \\
& =-\frac{m l^{2} \omega^{2}}{216}\left\{\left[4\left(1+k_{1}^{N}\right)+2\left(1+k_{2}^{N}\right)\right]^{2}+\left[4\left(1+k_{1}^{N}\right)+2\left(1+k_{2}^{N}\right)\right]\left[4\left(1+k_{2}^{N}\right)+2\left(1+k_{1}^{N}\right)\right]\right. \\
& \left.+\left[4\left(1+k_{2}^{N}\right)+2\left(1+k_{1}^{N}\right)\right]^{2}\right\}+\frac{m l^{2} \omega^{2}}{36}\left[6\left(1+k_{1}^{N}\right)+6\left(1+k_{2}^{N}\right)\right], \\
& {[\mathbf{D}]\left[\mathbf{M}_{r e d}\right]^{-1}[\mathbf{D}][\boldsymbol{\eta}]=\frac{1}{m J_{z}}\left[\begin{array}{cccccc}
0 & 0 & 0 & 0 & 0 & 0 \\
0 & 0 & 0 & 0 & 0 & 0 \\
0 & 0 & 0 & 0 & 0 & m \\
J_{z} & 0 & 0 & 0 & 0 & 0 \\
0 & 0 & 0 & 0 & 0 & 0 \\
0 & 0 & 0 & 0 & 0 & 0
\end{array}\right],} \\
& \left\{\mathbf{v}_{C}\right\}=\left\{\mathbf{v}_{C}^{0}\right\}-\sum_{i=1}^{2} P_{i}[\mathbf{D}]\left[\mathbf{M}_{r e d}\right]^{-1}[\mathbf{D}]^{\mathrm{T}}[\boldsymbol{\eta}]\left\{\mathbf{E}_{i x}\right\}=\left[\begin{array}{c}
0 \\
0 \\
-\frac{\omega\left(k_{1}^{N}+k_{2}^{N}\right)}{2 \omega}\left(k_{2}^{N}-k_{1}^{N}\right) \\
0 \\
0
\end{array}\right] \text {, }
\end{aligned}
$$$$
\left[[\mathbf{U}]^{\mathrm{T}}[\boldsymbol{\eta}][\mathbf{M}]^{-1}[\mathbf{U}]\right]^{-1}[\mathbf{U}]^{\mathrm{T}}[\boldsymbol{\eta}][\mathbf{M}]^{-1}\left\{\mathbf{E}_{1 x}\right\}=\left[\begin{array}{llll}
0 & 0 & 0 & 0
\end{array}\right]^{\mathrm{T}},\left[[\mathbf{U}]^{\mathrm{T}}[\boldsymbol{\eta}][\mathbf{M}]^{-1}[\mathbf{U}]\right]^{-1}[\mathbf{U}]^{\mathrm{T}}[\boldsymbol{\eta}][\mathbf{M}]^{-1}\left\{\mathbf{E}_{2 x}\right\}=\left[\begin{array}{llll}
0 & 0 & 0 & 0
\end{array}\right]^{\mathrm{T}} .
$$

As numerical values, we consider: $m=10 \mathrm{~kg}, l=1 \mathrm{~m}, \omega=1 \mathrm{rad} / \mathrm{s}$, and $a=b=\frac{l}{3}$. The simulations are performed in the following cases: (i) $k_{1}^{N}=0, k_{2}^{N}=0$; (ii) $k_{1}^{N}=0$, $k_{2}^{N}=0.5$; (iii) $k_{1}^{N}=0, k_{2}^{N}=1$; (iv) $k_{1}^{N}=0.5, k_{2}^{N}=0$; (v) $k_{1}^{N}=0.5, k_{2}^{N}=0.5$; (vi) $k_{1}^{N}=0.5$, $k_{2}^{N}=1$; (vii) $k_{1}^{N}=1, k_{2}^{N}=0$; (viii) $k_{1}^{N}=1, k_{2}^{N}=0.5$; (ix) $k_{1}^{N}=1, k_{2}^{N}=1$. The results are presented in Table 2.

Table 2. Results for the numerical cases considered in Example 6.

\begin{tabular}{cccccccccc}
\hline Case & (i) & (ii) & (iii) & (iv) & (v) & (vi) & (vii) & (viii) & (ix) \\
\hline$P_{1}[\mathrm{Ns}]$ & 5 & 5.833 & 6.667 & 6.667 & 7.5 & 8.333 & 8.333 & 9.167 & 10 \\
$P_{2}[\mathrm{Ns}]$ & -5 & -6.667 & -8.393 & -5.833 & -7.5 & -9.167 & -6.667 & -8.333 & -10 \\
$v_{1 x}[\mathrm{~m} / \mathrm{s}]$ & 0 & 0 & 0 & -0.167 & -0.167 & -0.167 & -0.333 & -0.333 & -0.333 \\
$v_{2 x}[\mathrm{~m} / \mathrm{s}]$ & 0 & 0.167 & 0.333 & 0 & 0.167 & 0.333 & 0 & 0.167 & 0.333 \\
$E_{p}[\mathrm{~J}]$ & 1.667 & 2.639 & 3.889 & 2.639 & 3.75 & 5.139 & 3.889 & 5.139 & 6.667 \\
$\Delta E_{c}[\mathrm{~J}]$ & 1.667 & 1.528 & 1.111 & 1.528 & 1.25 & 0.694 & 1.111 & 0.694 & 0 \\
\hline
\end{tabular}

Remark 2. One may solve this example considering the rigid solid as a free one to obtain the same results.

Indeed, we have

$$
[\mathbf{M}]^{-1}=\left[\begin{array}{cccccc}
0 & 0 & 0 & J_{x}^{-1} & 0 & 0 \\
0 & 0 & 0 & 0 & J_{y}^{-1} & 0 \\
0 & 0 & 0 & 0 & 0 & J_{z}^{-1} \\
m^{-1} & 0 & 0 & 0 & 0 & 0 \\
0 & m^{-1} & 0 & 0 & 0 & 0 \\
0 & 0 & m^{-1} & 0 & 0 & 0
\end{array}\right]
$$




$$
\begin{aligned}
\left\{\mathbf{E}_{1 x}\right\}=\left[\begin{array}{llllll}
1 & 0 & 0 & 0 & 0 & a
\end{array}\right]^{\mathrm{T}},\left\{\mathbf{E}_{2 x}\right\}=\left[\begin{array}{llllll}
1 & 0 & 0 & 0 & 0 & -b
\end{array}\right]^{\mathrm{T}}, \\
g_{11 x x}=\left\{\mathbf{E}_{1 x}\right\}^{\mathrm{T}}[\mathbf{\eta}][\mathbf{M}]^{-1}\left\{\mathbf{E}_{1 x}\right\}=\frac{I_{z}+m a^{2}}{m}, \\
g_{12 x x}=\left\{\mathbf{E}_{1 x}\right\}^{\mathrm{T}}[\mathbf{\eta}][\mathbf{M}]^{-1}\left\{\mathbf{E}_{2 x}\right\}=\frac{I_{z}-m a b}{m}, \\
g_{22 x x}=\left\{\mathbf{E}_{2 x}\right\}^{\mathrm{T}}[\mathbf{\eta}][\mathbf{M}]^{-1}\left\{\mathbf{E}_{2 x}\right\}=\frac{I_{z}+m b^{2}}{m} .
\end{aligned}
$$

The resulting values are the same for $P_{1}$, and $P_{2}$. Moreover, since

$$
\begin{aligned}
{[\mathbf{M}]^{-1}\left\{\mathbf{E}_{1 x}\right\} } & =[\mathbf{D}]\left[\mathbf{M}_{r e d}\right]^{-1}[\mathbf{D}]^{\mathrm{T}}[\boldsymbol{\eta}]\left\{\mathbf{E}_{1 x}\right\}=\left[\begin{array}{llllll}
0 & 0 & \frac{a}{J_{z}} & \frac{1}{m} & 0 & 0
\end{array}\right]^{\mathrm{T}}, \\
{[\mathbf{M}]^{-1}\left\{\mathbf{E}_{2 x}\right\} } & =[\mathbf{D}]\left[\mathbf{M}_{r e d}\right]^{-1}[\mathbf{D}]^{\mathrm{T}}[\boldsymbol{\eta}]\left\{\mathbf{E}_{2 x}\right\}=\left[\begin{array}{llllll}
0 & 0 & -\frac{b}{J_{z}} & \frac{1}{m} & 0 & 0
\end{array}\right]^{\mathrm{T}},
\end{aligned}
$$

one obtains the same value for $\left\{\mathbf{v}_{C}\right\}$.

The coincidence of the results is explained by the fact that the motion is permitted along the directions on which there exist the impulses.

Example 7. Let us assume that the only possibilities of motion for the bar are the rotation about the $\mathrm{OZ}$ axis, and the displacement along the straight line (d) (Figure 9). $v_{z}^{0}=0$

The initial conditions are $\omega_{x}^{0}=0, \omega_{y}^{0}=0, \omega_{z}^{0}=\omega, v_{x}^{0}=3 \omega l, v_{y}^{0}=4 \omega l \tan \alpha$, and

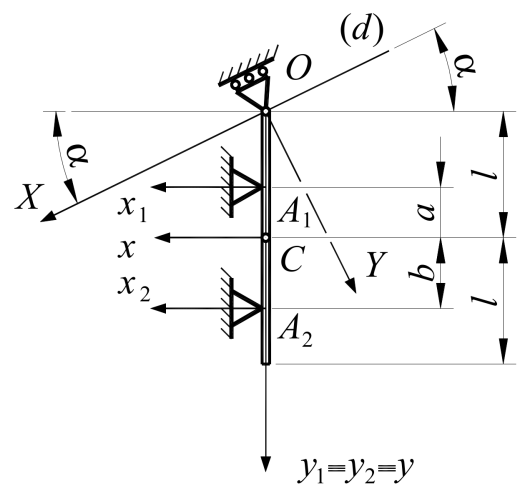

Figure 9. Example 7.

In this situation, we have

$\mathbf{C O}=-l \sin \alpha \mathbf{i}-l \cos \alpha \mathbf{j}, \mathbf{C O} \times \mathbf{i}=l \cos \alpha \mathbf{k}, \mathbf{C O} \times \mathbf{j}=-l \sin \alpha \mathbf{k}, \mathbf{C O} \times \mathbf{k}=l \sin \alpha \mathbf{j}-l \cos \alpha \mathbf{i}$,

$$
\begin{aligned}
& \left\{\mathbf{D}_{1}\right\}=\left[\begin{array}{llllll}
0 & 0 & 1 & -l \cos \alpha & l \sin \alpha & 0
\end{array}\right]^{\mathrm{T}},\left\{\mathbf{D}_{2}\right\}=\left[\begin{array}{llllll}
0 & 0 & 0 & 1 & 0 & 0
\end{array}\right]^{\mathrm{T}}, \\
& \left\{\mathbf{U}_{1}\right\}=\left[\begin{array}{llllll}
0 & 1 & 0 & 0 & 0 & -l \sin \alpha
\end{array}\right]^{\mathrm{T}},\left\{\mathbf{U}_{2}\right\}=\left[\begin{array}{llllll}
0 & 0 & 1 & -l \cos \alpha & l \sin \alpha & 0
\end{array}\right]^{\mathrm{T}}, \\
& \left\{\mathbf{U}_{3}\right\}=\left[\begin{array}{llllll}
0 & 0 & 0 & 1 & 0 & 0
\end{array}\right]^{\mathrm{T}},\left\{\mathbf{U}_{4}\right\}=\left[\begin{array}{llllll}
0 & 0 & 0 & 0 & 1 & 0
\end{array}\right]^{\mathrm{T}} \text {, } \\
& {[\mathbf{D}]=\left[\begin{array}{cc}
0 & 0 \\
0 & 0 \\
1 & 0 \\
-l & \cos \alpha \\
0 & \sin \alpha \\
0 & 0
\end{array}\right],[\mathbf{U}]=\left[\begin{array}{cccc}
-\sin \alpha & 0 & 0 & 0 \\
\cos \alpha & 0 & 0 & 0 \\
0 & 1 & 0 & 0 \\
0 & 0 & 1 & 0 \\
0 & 0 & 0 & 1 \\
-l \sin \alpha & 0 & 0 & 0
\end{array}\right],[\mathbf{D}]^{\mathrm{T}}[\mathbf{\eta}][\mathbf{U}]=\left[\begin{array}{llll}
0 & 0 & 0 & 0 \\
0 & 0 & 0 & 0
\end{array}\right]}
\end{aligned}
$$




$$
\begin{aligned}
& \left\{\mathbf{E}_{1 x}\right\}=\left[\begin{array}{llllll}
1 & 0 & 0 & 0 & 0 & a
\end{array}\right]^{\mathrm{T}},\left\{\mathbf{E}_{1 y}\right\}=\left[\begin{array}{llllll}
0 & 1 & 0 & 0 & 0 & 0
\end{array}\right]^{\mathrm{T}},\left\{\mathbf{E}_{1 z}\right\}=\left[\begin{array}{llllll}
0 & 0 & 1 & -a & 0 & 0
\end{array}\right]^{\mathrm{T}}, \\
& \left\{\mathbf{E}_{2 x}\right\}=\left[\begin{array}{llllll}
1 & 0 & 0 & 0 & 0 & -b
\end{array}\right]^{\mathrm{T}},\left\{\mathbf{E}_{2 y}\right\}=\left[\begin{array}{llllll}
0 & 1 & 0 & 0 & 0 & 0
\end{array}\right]^{\mathrm{T}},\left\{\mathbf{E}_{2 z}\right\}=\left[\begin{array}{llllll}
0 & 0 & 1 & b & 0 & 0
\end{array}\right]^{\mathrm{T}}, \\
& {\left[\mathbf{M}_{r e d}\right]=[\mathbf{D}]^{\mathrm{T}}[\mathbf{\eta}][\mathbf{M}][\mathbf{D}]=\left[\begin{array}{cc}
J_{z}+m l^{2} & -m l \cos \alpha \\
-m l \cos \alpha & m
\end{array}\right],\left[\mathbf{M}_{r e d}\right]^{-1}=\frac{1}{J_{z}+m l^{2} \sin ^{2} \alpha}\left[\begin{array}{cc}
m & m l \cos \alpha \\
m l \cos \alpha & J_{z}+m l^{2}
\end{array}\right]} \\
& \left\{\mathbf{v}_{C}^{0}\right\}=\left[\begin{array}{llllll}
0 & 0 & \omega & 3 \omega l & 4 \omega l \tan \alpha & 0
\end{array}\right]^{\mathrm{T}} \\
& v_{1 x}^{0}=\left\{\mathbf{E}_{1 x}\right\}^{\mathrm{T}}[\boldsymbol{\eta}]\left\{\mathbf{v}_{C}^{0}\right\}=\omega a+3 \omega l, v_{1 y}^{0}=\left\{\mathbf{E}_{1 y}\right\}^{\mathrm{T}}[\boldsymbol{\eta}]\left\{\mathbf{v}_{C}^{0}\right\}=4 \omega l \tan \alpha, v_{1 z}^{0}=\left\{\mathbf{E}_{1 z}\right\}^{\mathrm{T}}[\mathbf{\eta}]\left\{\mathbf{v}_{C}^{0}\right\}=0, \\
& v_{2 x}^{0}=\left\{\mathbf{E}_{2 x}\right\}^{\mathrm{T}}[\mathbf{\eta}]\left\{\mathbf{v}_{C}^{0}\right\}=-\omega b+3 \omega l, v_{2 y}^{0}=\left\{\mathbf{E}_{2 y}\right\}^{\mathrm{T}}[\mathbf{\eta}]\left\{\mathbf{v}_{C}^{0}\right\}=4 \omega l \tan \alpha, v_{2 z}^{0}=\left\{\mathbf{E}_{2 z}\right\}^{\mathrm{T}}[\boldsymbol{\eta}]\left\{\mathbf{v}_{C}^{0}\right\}=0
\end{aligned}
$$

The simulations are performed for $m=10 \mathrm{~kg}, l=1 \mathrm{~m}, \omega=1 \mathrm{rad} / \mathrm{s}, a=b=\frac{l}{3}$, and the following cases: (i) $k_{1}^{N}=0, k_{2}^{N}=0$; (ii) $k_{1}^{N}=0.3, k_{2}^{N}=0.7$; and (iii) $k_{1}^{N}=1, k_{2}^{N}=1$. The results are given in Table 3 for $\alpha=30^{\circ}$, Table 4 for $\alpha=45^{0}$, and Table 5 for $\alpha=60^{0}$.

Table 3. Results for the numerical cases considered in Example 7, $\alpha=30^{0}$.

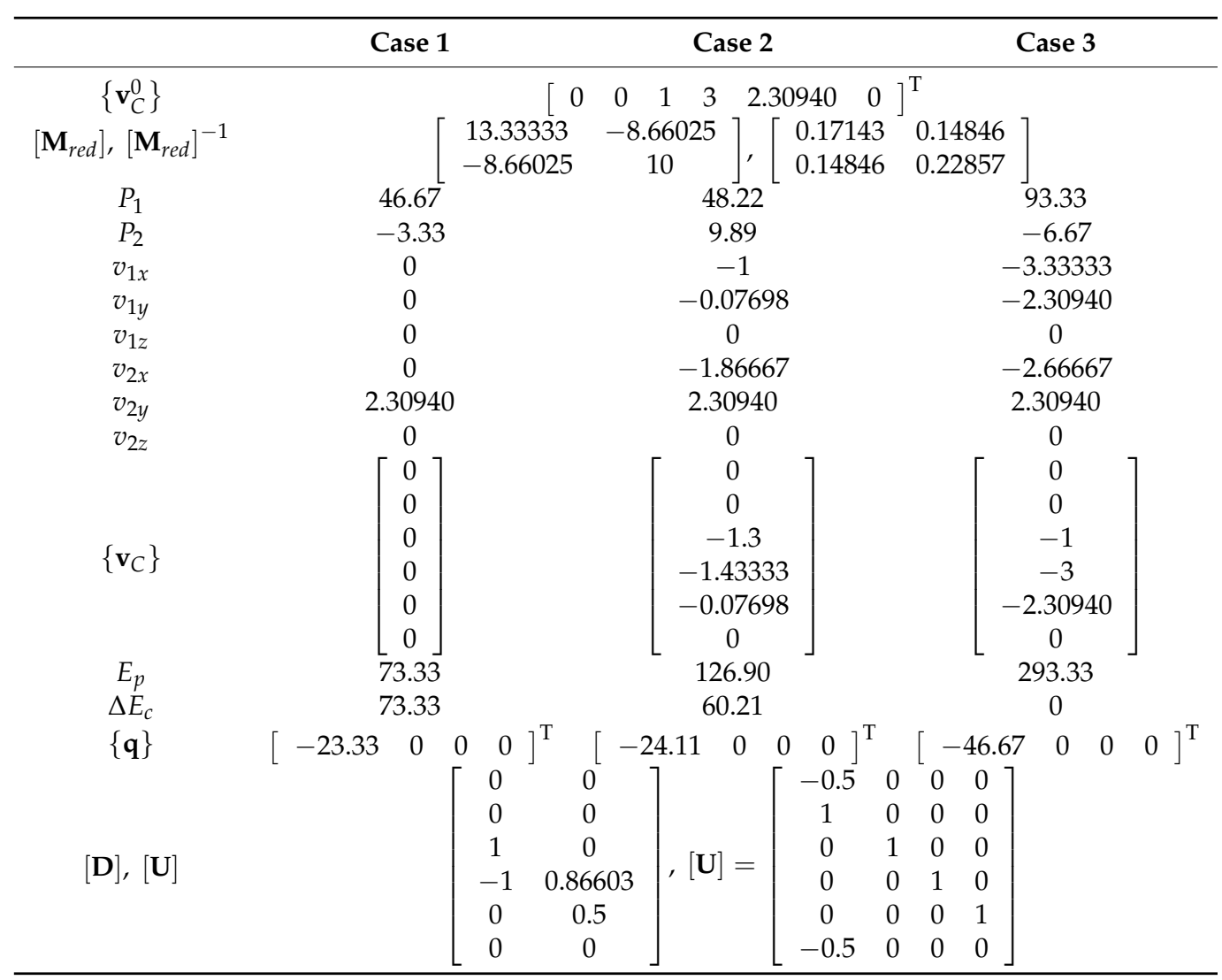


Table 4. Results for the numerical cases considered in Example 7, $\alpha=45^{0}$.

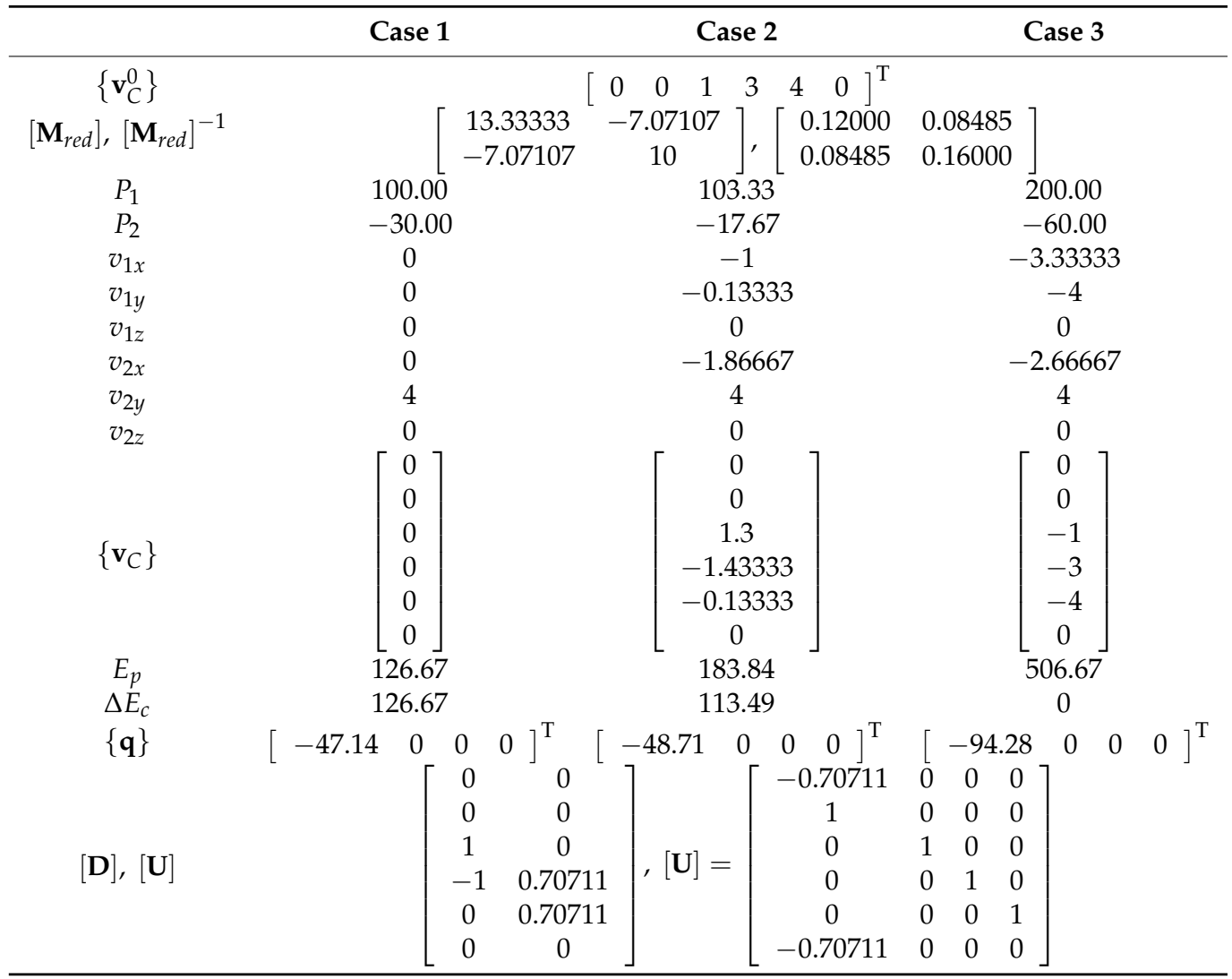

Table 5. Results for the numerical cases considered in Example 7, $\alpha=60^{\circ}$.

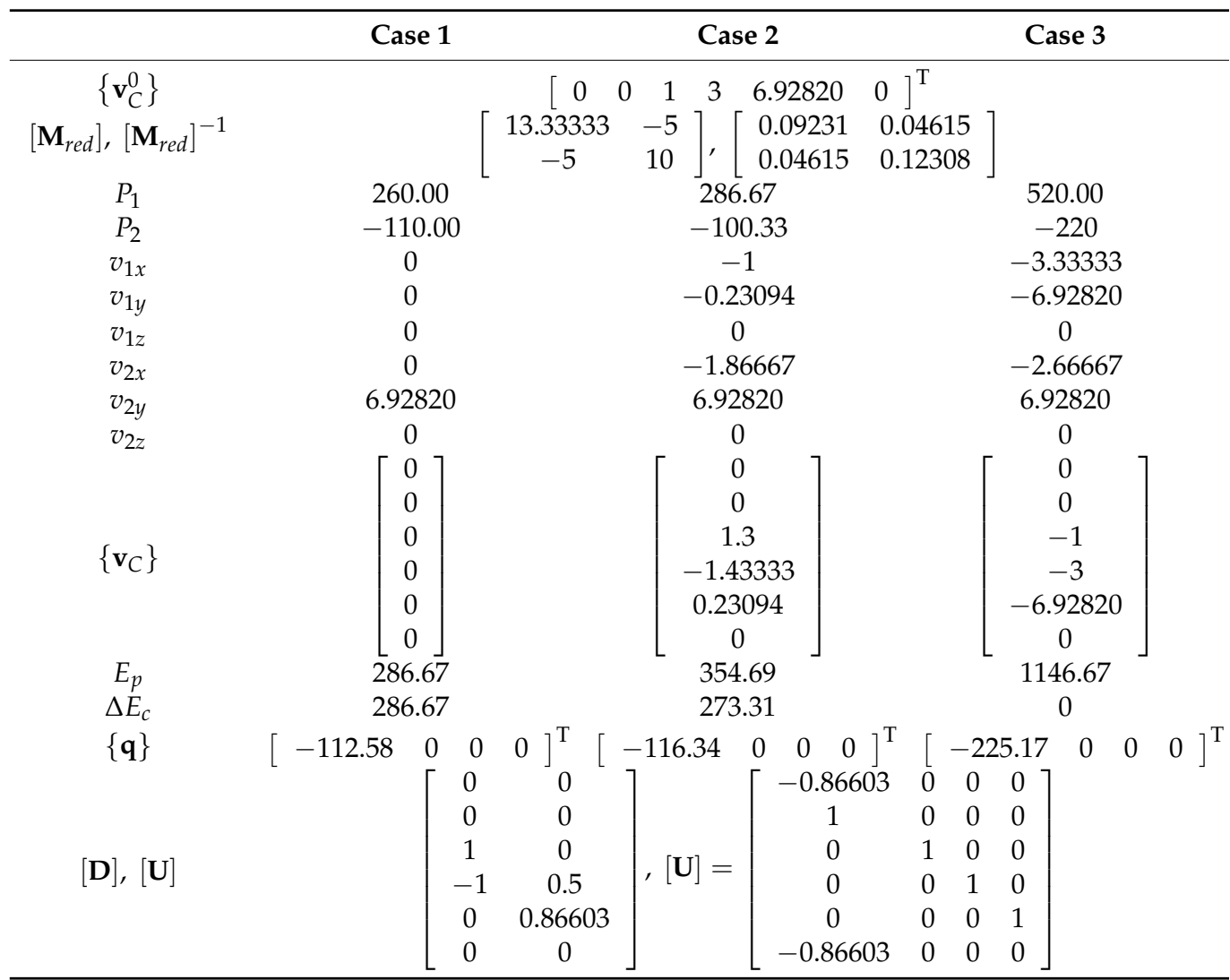




\section{Algorithm for the Collision at Two Points of the Rigid Solid Bounded by the} Surface of Equation $f(x, y, z)=0$

The algorithm is as follows:

Inputs:

- $\quad$ the geometric and mechanical parameters of the rigid solid;

- the initial velocity of the center of weight $\left\{\begin{array}{l}-0 \\ \mathbf{v}_{C}\end{array}\right\}=\left[\begin{array}{lll}v_{C x}^{0} & v_{C y}^{0} & v_{C z}^{0}\end{array}\right]^{\mathrm{T}}$;

- $\quad$ the coordinates $x_{i}, y_{i}$, and $z_{i}$ of the collision points $A_{i}, i=\overline{1,2}$;

- $\quad$ the coefficients of restitution $k_{1}, k_{2}$.

Step 1. Determination of the matrices $\left\{\mathbf{E}_{1 x}\right\}$ and $\left\{\mathbf{E}_{2 x}\right\}$ of the plückerian (screw) coordinates.

One successively calculates

$$
\begin{aligned}
& D f_{1 x}=\left.\frac{\partial f}{\partial x}\right|_{A_{1}}, D f_{1 y}=\left.\frac{\partial f}{\partial y}\right|_{A_{1}}, D f_{1 z}=\left.\frac{\partial f}{\partial z}\right|_{A_{1}}{ }^{\prime} \\
& D f_{2 x}=\left.\frac{\partial f}{\partial x}\right|_{A_{2}}, D f_{2 y}=\left.\frac{\partial f}{\partial y}\right|_{A_{2}}, D f_{2 z}=\left.\frac{\partial f}{\partial z}\right|_{A_{2}}{ }^{\prime} \\
& R D f_{1}=\sqrt{\left(D f_{1 x}\right)^{2}+\left(D f_{1 y}\right)^{2}+\left(D f_{1 z}\right)^{2}}, R D f_{2}=\sqrt{\left(D f_{2 x}\right)^{2}+\left(D f_{2 y}\right)^{2}+\left(D f_{2 z}\right)^{2}} \text {, } \\
& a_{11}=\frac{D f_{1 x}}{R D f_{1}}, b_{11}=\frac{D f_{1 y}}{R D f_{1}}, c_{11}=\frac{D f_{1 z}}{R D f_{1}}, \\
& a_{21}=\frac{D f_{2 x}}{R D f_{2}}, b_{21}=\frac{D f_{2 y}}{R D f_{2}}, c_{21}=\frac{D f_{2 z}}{R D f_{2}}, \\
& {\left[\begin{array}{l}
d_{11} \\
e_{11} \\
f_{11}
\end{array}\right]=\left[\begin{array}{ccc}
0 & -z_{1} & y_{1} \\
z_{1} & 0 & -x_{1} \\
-y_{1} & x_{1} & 0
\end{array}\right]\left[\begin{array}{l}
a_{11} \\
b_{11} \\
c_{11}
\end{array}\right],} \\
& {\left[\begin{array}{l}
d_{21} \\
e_{21} \\
f_{21}
\end{array}\right]=\left[\begin{array}{ccc}
0 & -z_{2} & y_{2} \\
z_{2} & 0 & -x_{2} \\
-y_{2} & x_{2} & 0
\end{array}\right]\left[\begin{array}{l}
a_{21} \\
b_{21} \\
c_{21}
\end{array}\right] \text {, }} \\
& \left\{\mathbf{E}_{1 x}\right\}=\left[\begin{array}{llllll}
a_{11} & b_{11} & c_{11} & d_{11} & e_{11} & f_{11}
\end{array}\right]^{\mathrm{T}} \text {, } \\
& \left\{\mathbf{E}_{2 x}\right\}=\left[\begin{array}{llllll}
a_{21} & b_{21} & c_{21} & d_{21} & e_{21} & f_{21}
\end{array}\right]^{\mathrm{T}} \text {. }
\end{aligned}
$$

Step 2. Determination of the matrices $\left\{\mathbf{E}_{1 y}\right\},\left\{\mathbf{E}_{2 y}\right\},\left\{\mathbf{E}_{1 z}\right\}$, and $\left\{\mathbf{E}_{2 z}\right\}$. One calculates:

$$
\begin{gathered}
{\left[\begin{array}{l}
V L_{i x}^{0} \\
V L_{i y}^{0} \\
V L_{i z}^{0}
\end{array}\right]=\left\{\begin{array}{l}
-0 \\
\mathbf{v}_{C}
\end{array}\right\}+\left[\begin{array}{ccc}
0 & -\omega_{z} & \omega_{y} \\
\omega_{z} & 0 & -\omega_{x} \\
-\omega_{y} & \omega_{x} & 0
\end{array}\right]\left[\begin{array}{l}
x_{i} \\
y_{i} \\
z_{i}
\end{array}\right], i=\overline{1,2},} \\
{\left[\begin{array}{l}
N V_{i x} \\
N V_{i y} \\
N V_{i z}
\end{array}\right]=\left[\begin{array}{ccc}
0 & -c_{i 1} & b_{i 1} \\
c_{i 1} & 0 & -a_{i 1} \\
-b_{i 1} & a_{i 1} & 0
\end{array}\right]\left[\begin{array}{l}
V L_{i x}^{0} \\
V L_{i y}^{0} \\
V L_{i z}^{0}
\end{array}\right], i=\overline{1,2},} \\
R N V_{i}=\sqrt{\left(N V_{i x}\right)^{2}+\left(N V_{i y}\right)^{2}+\left(N V_{i z}\right)^{2}}, i=\overline{1,2}, \\
a_{13}=\frac{N V_{1 x}}{R N V_{1}}, b_{13}=\frac{N V_{1 y}}{R N V_{1}}, c_{13}=\frac{N V_{1 z}}{R N V_{1}}, \\
a_{23}=\frac{N V_{2 x}}{R N V_{2}}, b_{23}=\frac{N V_{2 y}}{R N V_{2}}, c_{23}=\frac{N V_{2 z}}{R N V_{2}},
\end{gathered}
$$




$$
\begin{aligned}
& {\left[\begin{array}{l}
d_{i 3} \\
e_{i 3} \\
f_{i 3}
\end{array}\right]=\left[\begin{array}{ccc}
0 & -z_{i} & y_{i} \\
z_{i} & 0 & -x_{i} \\
-y_{i} & x_{i} & 0
\end{array}\right]\left[\begin{array}{c}
a_{i 3} \\
b_{i 3} \\
c_{i 3}
\end{array}\right], i=\overline{1,2},} \\
& \left\{\mathbf{E}_{1 z}\right\}=\left[\begin{array}{llllll}
a_{13} & b_{13} & c_{13} & d_{13} & e_{13} & f_{13}
\end{array}\right]^{\mathrm{T}}, \\
& \left\{\mathbf{E}_{2 z}\right\}=\left[\begin{array}{llllll}
a_{23} & b_{23} & c_{23} & d_{23} & e_{23} & f_{23}
\end{array}\right]^{\mathrm{T}} \text {, } \\
& {\left[\begin{array}{l}
a_{i 2} \\
b_{i 2} \\
c_{i 2}
\end{array}\right]=\left[\begin{array}{ccc}
0 & -c_{i 3} & b_{i 3} \\
c_{i 3} & 0 & -a_{i 3} \\
-b_{i 3} & a_{i 3} & 0
\end{array}\right]\left[\begin{array}{l}
a_{i 1} \\
b_{i 1} \\
c_{i 1}
\end{array}\right], i=\overline{1,2},} \\
& {\left[\begin{array}{l}
d_{i 2} \\
e_{i 2} \\
f_{i 2}
\end{array}\right]=\left[\begin{array}{ccc}
0 & -z_{i} & y_{i} \\
z_{i} & 0 & -x_{i} \\
-y_{i} & x_{i} & 0
\end{array}\right]\left[\begin{array}{l}
a_{i 2} \\
b_{i 2} \\
c_{i 2}
\end{array}\right], i=\overline{1,2},} \\
& \left\{\mathbf{E}_{1 y}\right\}=\left[\begin{array}{llllll}
a_{12} & b_{12} & c_{12} & d_{12} & e_{12} & f_{12}
\end{array}\right]^{\mathrm{T}}, \\
& \left\{\mathbf{E}_{2 y}\right\}=\left[\begin{array}{llllll}
a_{22} & b_{22} & c_{22} & d_{22} & e_{22} & f_{22}
\end{array}\right]^{\mathrm{T}} .
\end{aligned}
$$

Step 3. Determination of the initial velocities in local coordinates at the contact points $A_{1}$ and $A_{2}$.

One calculates:

$$
\begin{aligned}
& v_{1 x}^{0}=\left\{\mathbf{E}_{1 x}\right\}^{\mathrm{T}}[\mathbf{\eta}]\left\{\mathbf{v}_{C}^{0}\right\}, v_{1 y}^{0}=\left\{\mathbf{E}_{1 y}\right\}[\mathbf{\eta}]\left\{\mathbf{v}_{C}^{0}\right\}, v_{1 z}^{0}=\left\{\mathbf{E}_{1 z}\right\}[\boldsymbol{\eta}]\left\{\mathbf{v}_{C}^{0}\right\}, \\
& v_{2 x}^{0}=\left\{\mathbf{E}_{2 x}\right\}^{\mathrm{T}}[\boldsymbol{\eta}]\left\{\mathbf{v}_{C}^{0}\right\}, v_{2 y}^{0}=\left\{\mathbf{E}_{2 y}\right\}[\boldsymbol{\eta}]\left\{\mathbf{v}_{C}^{0}\right\}, v_{2 z}^{0}=\left\{\mathbf{E}_{2 z}\right\}[\boldsymbol{\eta}]\left\{\mathbf{v}_{C}^{0}\right\} .
\end{aligned}
$$

Remark 3. One must obtain $v_{1 z}^{0}=0$ and $v_{2 z}^{0}=0$.

Step 4. Determination of the inertances.

One uses the formulae:

$$
\begin{gathered}
g_{11 x x}=\left\{\mathbf{E}_{1 x}\right\}^{\mathrm{T}}[\boldsymbol{\eta}][\mathbf{M}]^{-1}\left\{\mathbf{E}_{1 x}\right\}, g_{12 x x}=\left\{\mathbf{E}_{1 x}\right\}^{\mathrm{T}}[\boldsymbol{\eta}][\mathbf{M}]^{-1}\left\{\mathbf{E}_{2 x}\right\}, \\
g_{22 x x}=\left\{\mathbf{E}_{2 x}\right\}^{\mathrm{T}}[\boldsymbol{\eta}][\mathbf{M}]^{-1}\left\{\mathbf{E}_{2 x}\right\}, \\
g_{11 y x}=\left\{\mathbf{E}_{1 y}\right\}^{\mathrm{T}}[\boldsymbol{\eta}][\mathbf{M}]^{-1}\left\{\mathbf{E}_{1 x}\right\}, g_{12 y x}=\left\{\mathbf{E}_{1 y}\right\}^{\mathrm{T}}[\boldsymbol{\eta}][\mathbf{M}]^{-1}\left\{\mathbf{E}_{2 x}\right\}, \\
g_{22 y x}=\left\{\mathbf{E}_{2 y}\right\}^{\mathrm{T}}[\boldsymbol{\eta}][\mathbf{M}]^{-1}\left\{\mathbf{E}_{2 x}\right\}, \\
g_{11 z x}=\left\{\mathbf{E}_{1 z}\right\}^{\mathrm{T}}[\boldsymbol{\eta}][\mathbf{M}]^{-1}\left\{\mathbf{E}_{1 x}\right\}, g_{12 z x}=\left\{\mathbf{E}_{1 z}\right\}^{\mathrm{T}}[\boldsymbol{\eta}][\mathbf{M}]^{-1}\left\{\mathbf{E}_{2 x}\right\}, \\
g_{22 z x}=\left\{\mathbf{E}_{2 z}\right\}^{\mathrm{T}}[\boldsymbol{\eta}][\mathbf{M}]^{-1}\left\{\mathbf{E}_{2 x}\right\} .
\end{gathered}
$$

Step 5. Determination of the impulses.

One obtains:

$$
P_{1}=\frac{\left|\begin{array}{ll}
\left(1+k_{1}^{N}\right) v_{1 x}^{0} & g_{12 x x} \\
\left(1+k_{2}^{N}\right) v_{2 x}^{0} & g_{22 x x}
\end{array}\right|}{\left|\begin{array}{ll}
g_{11 x x} & g_{12 x x} \\
g_{12 x x} & g_{22 x x}
\end{array}\right|}, P_{2}=\frac{\left|\begin{array}{ll}
g_{11 x x} & \left(1+k_{1}^{N}\right) v_{1 x}^{0} \\
g_{12 x x} & \left(1+k_{2}^{N}\right) v_{2 x}^{0}
\end{array}\right|}{\left|\begin{array}{ll}
g_{11 x x} & g_{12 x x} \\
g_{12 x x} & g_{22 x x}
\end{array}\right|} .
$$

Step 6. Determination of the velocities after collision.

One calculates:

$$
\begin{gathered}
v_{1 x}=-k_{1}^{N} v_{1 x}^{0}, v_{2 x}=-k_{2}^{N} v_{2 x}^{0} \\
v_{1 y}=v_{1 y}^{0}-P_{1} g_{11 y x}-P_{2} g_{12 y x}, v_{2 y}=v_{2 y}^{0}-P_{1} g_{12 y x}-P_{2} g_{12 y x}, \\
v_{1 z}=v_{1 z}^{0}-P_{1} g_{11 z x}-P_{2} g_{12 z x}, v_{2 z}=v_{2 z}^{0}-P_{1} g_{12 z x}-P_{2} g_{22 z x},
\end{gathered}
$$




$$
\begin{gathered}
\left\{\mathbf{v}_{C}\right\}=\left\{\mathbf{v}_{C}^{0}\right\}-P_{1}[\mathbf{M}]^{-1}\left\{\mathbf{E}_{1 x}\right\}-P_{2}[\mathbf{M}]^{-1}\left\{\mathbf{E}_{2 x}\right\}, \\
\cos \alpha_{1}=\frac{v_{1 y}}{\sqrt{v_{1 y}^{2}+v_{1 z}^{2}}}, \sin \alpha_{1}=\frac{v_{1 z}}{\sqrt{v_{1 y}^{2}+v_{1 z}^{2}}}, \cos \alpha_{2}=\frac{v_{2 y}}{\sqrt{v_{2 y}^{2}+v_{2 z}^{2}}}, \\
\sin \alpha_{2}=\frac{v_{2 z}}{\sqrt{v_{2 y}^{2}+v_{2 z}^{2}}} .
\end{gathered}
$$

Step 7. Determination of the energy of the lost velocities and the variation of the kinetic energy.

One uses the formulae:

$$
\begin{gathered}
E_{p}=\frac{1}{2}\left(P_{1}^{2} g_{11 x x}+2 P_{1} P_{2} g_{12 x x}+P_{2}^{2} g_{22 x x}\right), \\
\Delta E_{c}=-E_{p}+P v_{1 x}^{0}+P_{2} v_{2 x}^{0} .
\end{gathered}
$$

\section{Example}

Example 8. Let us consider the sphere of equation

$$
f(x, y, z)=x^{2}+y^{2}+z^{2}-R^{2}=0
$$

and mass $m$, which is collided at the points $A_{1}(R, 0,0)$ and $A_{2}(0, R, 0)$ (that is, $x_{1}=R, y_{1}=0$, $\left.z_{1}=0, x_{2}=0, y_{2}=R, z_{2}=0\right)$. One knows: $v_{C x}^{0}=v^{0}, v_{C y}^{0}=2 v^{0}, v_{C z}^{0}=0, \omega_{x}=0, \omega_{y}=0$, $\omega_{z}=0$, and the coefficients of restitution $k_{1}^{N}$ and $k_{2}^{N}$.

We have:

$$
\begin{aligned}
& \frac{\partial f}{\partial x}=2 x, \frac{\partial f}{\partial y}=2 y, \frac{\partial f}{\partial z}=2 z, \\
& D f_{1 x}=2 R, D f_{1 y}=0, D f_{1 z}=0, D f_{2 x}=0, D f_{2 y}=2 R, D f_{2 z}=0 \text {, } \\
& R D f_{1}=2 R, R D f_{2}=2 R, \\
& a_{11}=1, b_{11}=0, c_{11}=0, a_{21}=0, b_{21}=1, c_{21}=0 \text {, } \\
& {\left[\begin{array}{l}
d_{11} \\
e_{11} \\
f_{11}
\end{array}\right]=\left[\begin{array}{l}
0 \\
0 \\
0
\end{array}\right],\left[\begin{array}{l}
d_{21} \\
e_{21} \\
f_{21}
\end{array}\right]=\left[\begin{array}{l}
0 \\
0 \\
0
\end{array}\right],} \\
& \left\{\mathbf{E}_{1 x}\right\}=\left[\begin{array}{llllll}
1 & 0 & 0 & 0 & 0 & 0
\end{array}\right]^{\mathrm{T}},\left\{\mathbf{E}_{2 x}\right\}=\left[\begin{array}{llllll}
0 & 1 & 0 & 0 & 0 & 0
\end{array}\right]^{\mathrm{T}}, \\
& \left\{\begin{array}{l}
\mathbf{v}_{C} \\
\mathbf{u}
\end{array}\right\}=\left[\begin{array}{lll}
v^{0} & 2 v^{0} & 0
\end{array}\right]^{\mathrm{T}},\left\{\mathbf{v}_{C}^{0}\right\}=\left[\begin{array}{llllll}
0 & 0 & 0 & v^{0} & 2 v^{0} & 0
\end{array}\right]^{\mathrm{T}}, \\
& {\left[\begin{array}{c}
V L_{1 x}^{0} \\
V L_{1 y}^{0} \\
V L_{1 z}^{0}
\end{array}\right]=\left[\begin{array}{c}
v^{0} \\
2 v^{0} \\
0
\end{array}\right],\left[\begin{array}{c}
V L_{2 x}^{0} \\
V L_{2 y}^{0} \\
V L_{2 z}^{0}
\end{array}\right]=\left[\begin{array}{c}
v^{0} \\
2 v^{0} \\
0
\end{array}\right],} \\
& {\left[\begin{array}{l}
N V_{1 x} \\
N V_{1 y} \\
N V_{1 z}
\end{array}\right]=\left[\begin{array}{c}
0 \\
0 \\
2 v^{0}
\end{array}\right],\left[\begin{array}{l}
N V_{2 x} \\
N V_{2 y} \\
N V_{2 z}
\end{array}\right]=\left[\begin{array}{c}
0 \\
0 \\
-v^{0}
\end{array}\right],} \\
& R N V_{1}=2 v^{0}, R N V_{2}=v^{0}, \\
& a_{13}=0, b_{13}=0, c_{13}=1, a_{23}=0, b_{23}=0, c_{23}=-1 \text {, } \\
& {\left[\begin{array}{l}
d_{13} \\
e_{13} \\
f_{13}
\end{array}\right]=\left[\begin{array}{c}
0 \\
-R \\
0
\end{array}\right],\left[\begin{array}{l}
d_{23} \\
e_{23} \\
f_{23}
\end{array}\right]=\left[\begin{array}{c}
-R \\
0 \\
0
\end{array}\right],} \\
& \left\{\mathbf{E}_{1 z}\right\}=\left[\begin{array}{llllll}
0 & 0 & 1 & 0 & -R & 0
\end{array}\right]^{\mathrm{T}},\left\{\mathbf{E}_{2 z}\right\}=\left[\begin{array}{llllll}
0 & 0 & -1 & -R & 0 & 0
\end{array}\right]^{\mathrm{T}} \text {, }
\end{aligned}
$$




$$
\begin{aligned}
& {\left[\begin{array}{l}
a_{12} \\
b_{12} \\
c_{12}
\end{array}\right]=\left[\begin{array}{l}
0 \\
1 \\
0
\end{array}\right],\left[\begin{array}{l}
a_{22} \\
b_{22} \\
c_{22}
\end{array}\right]=\left[\begin{array}{l}
1 \\
0 \\
0
\end{array}\right],\left[\begin{array}{l}
d_{12} \\
e_{12} \\
f_{12}
\end{array}\right]=\left[\begin{array}{l}
0 \\
0 \\
R
\end{array}\right],\left[\begin{array}{l}
d_{22} \\
e_{22} \\
f_{22}
\end{array}\right]=\left[\begin{array}{c}
0 \\
0 \\
-R
\end{array}\right],} \\
& \left\{\mathbf{E}_{1 y}\right\}=\left[\begin{array}{llllll}
0 & 1 & 0 & 0 & 0 & R
\end{array}\right]^{\mathrm{T}},\left\{\mathbf{E}_{2 y}\right\}=\left[\begin{array}{llllll}
1 & 0 & 0 & 0 & 0 & -R
\end{array}\right]^{\mathrm{T}} \text {, } \\
& v_{1 x}^{0}=v^{0}, v_{1 y}^{0}=2 v^{0}, v_{1 z}^{0}=0, v_{2 x}^{0}=2 v^{0}, v_{2 y}^{0}=v^{0}, v_{2 z}^{0}=0, \\
& J_{x}=J_{y}=J_{z}=J=\frac{2 m R^{2}}{5}, \\
& {[\mathbf{M}]=\left[\begin{array}{cccccc}
0 & 0 & 0 & m & 0 & 0 \\
0 & 0 & 0 & 0 & m & 0 \\
0 & 0 & 0 & 0 & 0 & m \\
J & 0 & 0 & 0 & 0 & 0 \\
0 & J & 0 & 0 & 0 & 0 \\
0 & 0 & J & 0 & 0 & 0
\end{array}\right],[\mathbf{M}]^{-1}=\left[\begin{array}{cccccc}
0 & 0 & 0 & J^{-1} & 0 & 0 \\
0 & 0 & 0 & 0 & J^{-1} & 0 \\
0 & 0 & 0 & 0 & 0 & J^{-1} \\
m^{-1} & 0 & 0 & 0 & 0 & 0 \\
0 & m^{-1} & 0 & 0 & 0 & 0 \\
0 & 0 & m^{-1} & 0 & 0 & 0
\end{array}\right],} \\
& g_{11 x x}=\frac{1}{m}, g_{12 x x}=0, g_{22 x x}=\frac{1}{m}, g_{11 y x}=0, g_{12 y x}=\frac{1}{m}, g_{22 y x}=0, g_{11 z x}=0 \text {, } \\
& g_{12 z x}=0, g_{22 z x}=0, \\
& P_{1}=m v^{0}\left(1+k_{1}^{N}\right), P_{2}=2 m v^{0}\left(1+k_{2}^{N}\right), \\
& v_{1 x}=-k_{1}^{N} v^{0}, v_{2 x}^{0}=-2 k_{2}^{N} v^{0}, v_{1 y}=-2 k_{2}^{N} v^{0}, v_{2 y}=-k_{1}^{N} v^{0}, v_{1 z}=0, v_{2 z}=0 \text {, } \\
& \cos \alpha_{1}=-1, \sin \alpha_{1}=0, \alpha_{1}=180^{\circ} \text {, } \\
& \cos \alpha_{2}=-1, \sin \alpha_{2}=0, \alpha_{2}=180^{\circ} \text {, } \\
& \left\{\mathbf{v}_{C}\right\}=\left[\begin{array}{llllll}
0 & 0 & 0 & -k_{1}^{N} v^{0} & -2 k_{2}^{N} v^{0} & 0
\end{array}\right]^{\mathrm{T}} \text {, } \\
& E_{p}=\frac{m\left(v^{0}\right)^{2}}{2}\left[\left(1+k_{1}^{N}\right)^{2}+4\left(1+k_{2}^{N}\right)^{2}\right], \\
& \Delta E_{c}=\frac{m\left(v^{0}\right)^{2}}{2}\left[5-\left(k_{1}^{N}\right)^{2}-4\left(k_{2}^{N}\right)^{2}\right] .
\end{aligned}
$$

\section{Collision with Coulombian Friction}

\subsection{General Aspects}

In the case of collision with friction, one has to take into account the existence of the tangential impulses $\mathbf{P}_{i t}$, which are situated in the planes $A_{i} y_{i} z_{i}$ (Figure 10). The scalar values of these impulses are $\mu_{i} P_{i}, i=\overline{1,2}$, while their senses are opposite to the tangential velocities $\mathbf{v}_{i t}$. The scalar components of the tangential velocities read $v_{i y}$ and $v_{i z}, i=\overline{1,2}$.

Consequently, the impulses at the points $A_{i}, i=\overline{1,2}$, have the components $P_{i}$, $\mu_{i} P_{i} \cos \alpha_{i}$, and $\mu_{i} P_{i} \sin \alpha_{i}$, where

$$
\cos \alpha_{i}=\frac{v_{i y}}{\sqrt{v_{i y}^{2}+v_{i z}^{2}}}, \sin \alpha_{i}=\frac{v_{i z}}{\sqrt{v_{i y}^{2}+v_{i z}^{2}}}, i=\overline{1,2} .
$$

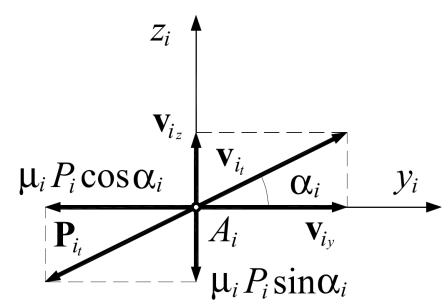

Figure 10. Scalar components of impulse and velocity. 


\subsection{Mathematical Model}

Taking into account the components of the impulses, the general theorems $[13,14,48,49]$ read

$$
[\mathbf{M}]\left\{\left\{\mathbf{v}_{C}\right\}-\left\{\mathbf{v}_{C}^{0}\right\}\right\}=-\sum_{i=1}^{2} P_{i}\left\{\left\{\mathbf{E}_{i x}\right\}+\mu_{i} \cos \alpha_{i}\left\{\mathbf{E}_{i y}\right\}+\mu_{i} \sin \alpha_{i}\left\{\mathbf{E}_{i z}\right\}\right\} .
$$

Using the notations:

$$
\begin{gathered}
\left\{\boldsymbol{\alpha}_{i}\right\}=\left[\begin{array}{lll}
1 & \mu_{i} \cos \alpha_{i} & \mu_{i} \sin \alpha_{i}
\end{array}\right]^{\mathrm{T}}, i=\overline{1,2}, \\
{\left[\begin{array}{c}
{\left[\mathbf{R}_{i}\right]} \\
{\left[\mathbf{D}_{i}\right]}
\end{array}\right]=\left[\begin{array}{lll}
\left\{\mathbf{E}_{i x}\right\} & \left\{\mathbf{E}_{i y}\right\} & \left\{\mathbf{E}_{i z}\right\}
\end{array}\right],}
\end{gathered}
$$

one obtains

$$
[\mathbf{M}]\left\{\left\{\mathbf{v}_{C}\right\}-\left\{\mathbf{v}_{C}^{0}\right\}\right\}=-\sum_{i=1}^{2} P_{i}\left[\begin{array}{c}
{\left[\mathbf{R}_{i}\right]} \\
{\left[\mathbf{D}_{i}\right]}
\end{array}\right]\left\{\boldsymbol{\alpha}_{i}\right\},
$$

wherefrom

$$
\left\{\left\{\mathbf{v}_{C}\right\}-\left\{\mathbf{v}_{C}^{0}\right\}\right\}=-\sum_{i=1}^{2} P_{i}[\mathbf{M}]^{-1}\left[\begin{array}{c}
{\left[\mathbf{R}_{i}\right]} \\
{\left[\mathbf{D}_{i}\right]}
\end{array}\right]\left\{\boldsymbol{\alpha}_{i}\right\} .
$$

We denote by $\left[\mathbf{H}_{i x}\right],\left[\mathbf{H}_{i y}\right]$, and $\left[\mathbf{H}_{i z}\right], i=\overline{1,2}$, the matrices of inertances,

$$
\begin{gathered}
{\left[\mathbf{H}_{i x}\right]=\left\{\mathbf{E}_{i x}\right\}^{\mathrm{T}}[\boldsymbol{\eta}][\mathbf{M}]^{-1}\left[\begin{array}{c}
{\left[\mathbf{R}_{i}\right]} \\
{\left[\mathbf{D}_{i}\right]}
\end{array}\right],\left[\mathbf{H}_{i y}\right]=\left\{\mathbf{E}_{i y}\right\}^{\mathrm{T}}[\boldsymbol{\eta}][\mathbf{M}]^{-1}\left[\begin{array}{c}
{\left[\mathbf{R}_{i}\right]} \\
{\left[\mathbf{D}_{i}\right]}
\end{array}\right],} \\
{\left[\mathbf{H}_{i z}\right]=\left\{\mathbf{E}_{i z}\right\}^{\mathrm{T}}[\boldsymbol{\eta}][\mathbf{M}]^{-1}\left[\begin{array}{c}
{\left[\mathbf{R}_{i}\right]} \\
{\left[\mathbf{D}_{i}\right]}
\end{array}\right] .}
\end{gathered}
$$

These matrices are constant, each one having one row and three columns.

From the matrix relation (142), one deduces the system

$$
v_{i x}=v_{i x}^{0}-\sum_{i=1}^{2} P_{i}\left[\mathbf{H}_{i x}\right]\left\{\boldsymbol{\alpha}_{i}\right\}, v_{i y}=v_{i y}^{0}-\sum_{i=1}^{2} P_{i}\left[\mathbf{H}_{i y}\right]\left\{\boldsymbol{\alpha}_{i}\right\}, v_{i z}=v_{i z}^{0}-\sum_{i=1}^{2} P_{i}\left[\mathbf{H}_{i z}\right]\left\{\boldsymbol{\alpha}_{i}\right\},
$$

The system has six non-linear equations with eight unknowns $\left(v_{i x}, v_{i y}, v_{i z}, i=\overline{1,2}\right.$, $P_{1}$, and $P_{2}$ ).

The non-linearity is given by the relations (137), (139), and (144).

We have to work with differentials, the system (144) becoming

$$
\frac{\mathrm{d}}{\mathrm{d} P_{i}}\left[\begin{array}{c}
v_{i x} \\
v_{i y} \\
v_{i z}
\end{array}\right]=-\left[\begin{array}{c}
\sum_{i=1}^{2}\left[\mathbf{H}_{i x}\right]\left\{\boldsymbol{\alpha}_{i}\right\} \\
\sum_{i=1}^{2}\left[\mathbf{H}_{i y}\right]\left\{\boldsymbol{\alpha}_{i}\right\} \\
\sum_{i=1}^{2}\left[\mathbf{H}_{i z}\right]\left\{\boldsymbol{\alpha}_{i}\right\}
\end{array}\right] .
$$

\section{Conclusions}

This paper solves the problem of the simultaneous collision of a rigid solid at two points. The authors considered a supplementary hypothesis which states that the normal velocities at the contact points vanish simultaneously. The calculations are performed using the theory of the screw coordinates and the notion of inertances. We obtained the velocities after the collision, the energy of lost velocities, and the loss of kinetic energy. The problem is solved in both cases: free rigid solid and rigid solid with constraints. The coefficients of restitution are considered in the Newton and Poisson cases. We proved that in the general case, the results obtained using Newton and Poisson coefficients of 
restitution are different. In the case of the constrained rigid solid, we also deduced the expressions of the constraint impulses.

All the results are given in matrix form, and we present the algorithms of calculation in all possible situations. There are also cases of indetermination of the problem. The results are validated in some particular cases, proving that the problems may also be solved by classical methods.

Future works will be dedicated to the collision at several points of the rigid solid and to the case of the collisions with friction.

Author Contributions: Conceptualization, I.-B.D., N.P., and N.-D.S.; methodology, I.-B.D., N.P., and N.-D.S.; software, I.-B.D., N.P., and N.-D.S.; validation, I.-B.D., N.P., and N.-D.S.; formal analysis, I.-B.D., N.P., and N.-D.S.; investigation, I.-B.D., N.P., and N.-D.S.; resources, I.-B.D., N.P., and N.D.S.; data curation, I.-B.D., N.P., and N.-D.S.; writing-original draft preparation, I.-B.D., N.P., and N.-D.S.; writing-review and editing, I.-B.D., N.P., and N.-D.S.; visualization, I.-B.D., N.P., and N.-D.S.; supervision, I.-B.D., N.P., and N.-D.S.; project administration, I.-B.D., N.P., and N.-D.S.; funding acquisition, I.-B.D., N.P., and N.-D.S. All authors have read and agreed to the published version of the manuscript.

Funding: This research received no external funding.

Institutional Review Board Statement: Not applicable.

Informed Consent Statement: Not applicable.

Data Availability Statement: Not applicable.

Conflicts of Interest: The authors declare no conflict of interest.

\section{References}

1. Shi, P. The Restitution Coefficient for a Linear Elastic Rod. Math. Comput. Model. 1998, 28, 427-435. [CrossRef]

2. Shan, H.; Su, J.; Badiu, F.; Zhu, J.; Xu, L. Modeling and simulation of multiple impacts of falling rigid bodies. Math. Comput. Model. 2006, 43, 592-611. [CrossRef]

3. Rossikhin, Y.A.; Shitikova, M.V. The analysis of the impact response of a thin plate via fractional derivative standard linear solid model. J. Sound Vib. 2011, 330, 1985-2003. [CrossRef]

4. Chatterjee, A. On the Realism of Complementarity Conditions in Rigid Body Collisions. Nonlinear Dyn. 1999, $20,159-168$. [CrossRef]

5. Dal Pont, S.; Dimnet, E. A theory for multiple collisions of rigid solids and numerical simulation of granular flow. Int. J. Solids Struct. 2006, 43, 6100-6114. [CrossRef]

6. Pazouki, A.; Mazhar, H.; Negrut, D. Parallel collision detection of ellipsoids with applications in large scale multibody dynamics. Math. Comput. Simul. 2012, 82, 879-894. [CrossRef]

7. Rodgers, B.; Goyal, S.; Kelly, G.; Sheehy, M. The dynamics of multiple pair-wise collisions in a chain for designing optimal shock amplifiers. Shock Vib. 2009, 16, 99-116. [CrossRef]

8. Cheng, Z.; Pellettiere, J.A.; Crandall, J.R.; Pilkey, W.D. Optimal occupant kinematics and crash pulse for automobile frontal impact. Shock Vib. 2009, 16, 61-73. [CrossRef]

9. Batista, M. Self-induced jumping of a rigid body of revolution on a smooth horizontal surface. Int. J. Non-Linear Mech. 2008, 43, 26-35. [CrossRef]

10. Mao, J.; Fu, Y. Dynamics of Periodic Impulsive Collision in Escapement Mechanism. Shock Vib. 2013, 20, 1001-1010. [CrossRef]

11. Anisimov, A.V.; Zabaryanskii, S.F. Simulation of motion of a collection of rigid bodies, taking into account their collisions and joints. Cybern. Syst. Anal. 2005, 41, 500-508. [CrossRef]

12. Kapoulitsas, G.M. On the collision of rigid bodies. Z. Angew. Math. Phys. ZAMP 1995, 46, 709-723. [CrossRef]

13. Pandrea, N. About collisions of two solids with constraints. Rev. Romaine Sci. Tech. Sér. Méch. Appl. 2004, 49, 1-6.

14. Pandrea, N.; Stănescu, N.D. A New Approach in the Study of Frictionless Collisions Using Inertances. Proc. Int. Inst. Mech. Eng. Part C J. Mech. Eng. Sci. 2015, 229, 2144-2157. [CrossRef]

15. Pfeiffer, F. On impacts with friction. Appl. Math. Comput. 2010, 217, 1184-1192. [CrossRef]

16. Lankarani, H.M.; Fereira, M.F.O.S. Treatment of Impact with Friction in Planar Multibody Mechanical Systems. Multibody Syst. Dyn. 2001, 6, 203-227. [CrossRef]

17. Zhao, Z.; Liu, C.; Chen, B. The numerical method for three-dimensional impact with friction of multi-rigid-body system. Sci. China Ser. G Phys. Math. Astron. 2006, 49, 102-118. [CrossRef]

18. Yao, W.; Chen, B.; Liu, C. Energetic coefficient of restitution for planar impact in multi-rigid-body systems with friction. Int. J. Impact Eng. 2005, 31, 255-265. [CrossRef] 
19. Payr, M.; Glocker, C. Oblique Frictional Impact of a Bar: Analysis and Comparison of Different Impact Laws. Nonlinear Dyn. 2005, 41, 361-383. [CrossRef]

20. Nordmark, A.; Dankowicz, H.; Champneys, A. Discontinuity-induced bifurcations in systems with impacts and friction: Discontinuities in the impact law. Int. J. Non-Linear Mech. 2009, 44, 1011-1023. [CrossRef]

21. Shen, Y.; Stronge, W.J. Painlevé paradox during oblique impact with friction. Eur. J. Mech. A Solids 2011, 30, 457-467. [CrossRef]

22. Marghitu, D.B.; Hurmuzlu, Y. Nonlinear Dynamics of an Elastic Rod with Frictional Impact. Nonlinear Dyn. 1996, 10, 187-201. [CrossRef]

23. Battle, J.A. The Sliding Velocity Flow of Rough Collisions in Multibody Systems. J. Appl. Mech. 1996, 63, 804-809. [CrossRef]

24. Battle, J.A.; Cardona, S. The Jamb (Self-Locking) Process in Three-Dimensional Collisions. J. Appl. Mech. 1998, 65, 417-423. [CrossRef]

25. Battle, J.A. Termination conditions for three-dimensional inelastic collisions in multibody systems. Int. J. Impact Eng. 2001, 25, 615-629. [CrossRef]

26. Brach, R.M. Friction, Restitution, and Energy Loss in Planar Collisions. J. Appl. Mech. 1984, 51, 164-170. [CrossRef]

27. Brach, R.M. Rigid Body Collisions. J. Appl. Mech. 1989, 56, 133-138. [CrossRef]

28. Brogliato, B. Kinetic quasi-velocities in unilaterally constrained Lagrangian mechanics with impacts and friction. Multibody Syst. Dyn. 2013, 32, 175-216. [CrossRef]

29. Elkaranshawy, H.A. Rough collision in three-dimensional rigid multi-body systems. Proc. Inst. Mech. Eng. Part K J. Multi-Body Dyn. 2007, 221, 541-550. [CrossRef]

30. Glocker, C. Energetic consistency conditions for standard impacts. Part I: Newton type inequality impact laws and Kane's example. Multibody Syst. Dyn. 2013, 29, 77-117. [CrossRef]

31. Glocker, C. Energetic consistency conditions for standard impacts. Part II: Poisson-type inequality impact laws. Multibody Syst. Dyn. 2014, 32, 445-509. [CrossRef]

32. Keller, J.B. Impact with Friction. J. Appl. Mech. 1986, 53, 1-4. [CrossRef]

33. Marghitu, D.B.; Hurmuzlu, Y. Three-Dimensional Rigid-Body Collisions with Multiple Contact Points. J. Appl. Mech. 1995, 62, 725-732. [CrossRef]

34. Stronge, W.J. Rigid Body Collisions with Friction. Proc. R. Soc. A Math. Phys. Eng. Sci. 1990, 431, 169-181. [CrossRef]

35. Tavakoli, A.; Gharib, M.; Hurmuzlu, Y. Collision of Two Mass Baton with Massive External Surfaces. J. Appl. Mech. 2012, 79, 051019. [CrossRef]

36. Wang, Y.; Mason, M.T. Two-Dimensional Rigid-Body Collisions with Friction. J. Appl. Mech. 1992, 59, 635-642. [CrossRef]

37. Yu, H.N.; Zhao, J.S.; Chu, F.L. An enhanced multi-point dynamics methodology for collision and contact problem. Proc. Inst. Mech. Eng. Part C J. Mech. Eng. Sci. 2013, 227, 1203-1223. [CrossRef]

38. Chatterjee, A.; Rodriguez, A.; Bowling, A. Analytic solution for the planar indeterminate impact problems using an energy constraint. Multibody Syst. Dyn. 2018, 42, 347-379. [CrossRef]

39. Djerassi, S. Collision with friction; Part A: Newton's hypothesis. Multibody Syst. Dyn. 2009, 21, 37-54. [CrossRef]

40. Djerassi, S. Collision with friction; Part B: Poisson's and Stronge's hypotheses. Multibody Syst. Dyn. 2009, 21, 55-70. [CrossRef]

41. Djerassi, S. Stronge's hypothesis-based solution to the planar collision-with-friction problem. Multibody Syst. Dyn. 2010, 24, 493-515. [CrossRef]

42. Flores, P.; Ambrósio, J.; Claro, J.C.P.; Lankarani, H.M. Influence of the contact-impact force model on the dynamic response of multi-body systems. Proc. Inst. Mech. Eng. Part K J. Multi-Body Dyn. 2006, 220, 21-34. [CrossRef]

43. Pandrea, N.; Stănescu, N.D. A new approach in the study of the collisions with friction using inertances. Proc. Int. Inst. Mech. Eng. Part C J. Mech. Eng. Sci. 2019, 233, 817-834. [CrossRef]

44. Pennestrì, E.; Valentini, P.P.; Vita, L. Multibody dynamics simulation of planar linkages with Dahl friction. Multibody Syst. Dyn. 2007, 17, 321-347. [CrossRef]

45. Yuan, M.S.C.; Freudenstein, F. Kinematic Analysis of Spatial Mechanisms by Means of Screw Coordinates. Part 1-Screw Coordinates. J. Eng. Ind. 1971, 93, 61-66. [CrossRef]

46. Yuan, M.S.C.; Freudenstein, F.; Woo, L.S. Kinematic Analysis of Spatial Mechanisms by Means of Screw Coordinates. Part 2-Analysis of Spatial Mechanisms. J. Eng. Ind. 1971, 93, 67-73. [CrossRef]

47. Brogliato, B. Nonsmooth Mechanics, 3rd ed.; Springer: Berlin/Heidelberg, Germany, 2016.

48. Pandrea, N. Elements of the Mechanics of Solid Rigid in Plïckerian Coordinates; The Publishing House of the Romanian Academy: Bucharest, Romania, 2000.

49. Stănescu, N.D.; Munteanu, L.; Chiroiu, V.; Pandrea, N. Dynamical Systems. Theory and Applications; The Publishing House of the Romanian Academy: Bucharest, Romania, 2007. 